SAO Special Report No. 164

\title{
ANALYTICAL DEVELOPMENT OF THE PLANETARY DISTURBING FUNCTION ON A DIGITAL COMPUIER
}

\author{
by
}

I. G. Izsak, B. Benima, and Sara B. Mills

\author{
Smithsonian Institution \\ Astrophysical Observatory \\ Cambridge, Massachusetts 02138
}


TABLE OF CONTENTS

Introduction

1

1. The principal part

3

2. The complementary part

4

3. The pattern of indices

6

4. The program

References

5. Appendix I

21

6. Appendix II

61

Errata to SAO Special Report No. 140 


\section{ANALYTICAL DEVELOPMENT OF THE PLANETARY DISTURBING}

FUNCTION ON A DIGTPAL COMPUTER ${ }^{1}$

by

I. G. Izsak, ${ }^{2}$ B. Benima, ${ }^{3}$ and Sara B. Mills ${ }^{3}$

Introduction

The purpose of this report is to describe our recently completed IBM 7094 computer program for the analytical development of the planetary disturbing function. Our reason for undertaking this work and the mathematics of the program has already been documented in sufficient detail (Izsak and Benima, 1963; Izsak, Gerard, Efimba, and Barnett, 1964) We are now concerned with implementing the construction of a powerful computational tool that has various applications in celestial mechanics.

A few equations have to be added to those given in SAO Special Report No. 140, and their numbering is continued in this report. There will be a small change in notation. The choice of the indices $m_{1}$ and $m_{2}$ in the series (30) and in related formulas was unfortunate inasmuch as the same symbols were used to denote the masses of the planets. In this report these indices are marked with $s_{1}$ and $s_{2}$. The adopted expansion of the Jacobi coefficients is the classical one (12). In order to assure greater flexibility, our program does not use equations (28) and (29). Instead, the complementary part of the disturbing function is obtained separately from the principal one as follows.

The planetary disturbing function takes slightly different forms depending on the chosen coordinates in the basic differential equations of motion. Such a choice also affects the most convenient definition of orbital elements. Three possibilities of distinct advantage offer themselves: conventional relative coordinates, canonical relative coordinates, and Jacobi's canonical coordinates (Charlier, 1927). Only the first two will be considered here.

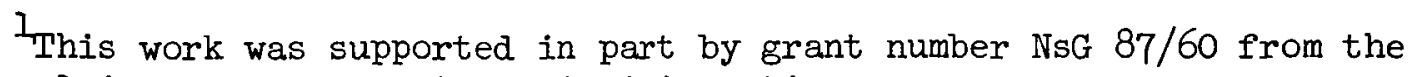
National Aeronautics and Space Administration.

Research and Analysis Division, Smithsonian Astrophysical Observatory, Cambridge, Massachusetts.

${ }^{3}$ Computations Division, Smithsonian Astrophysical observatory. 
Working with conventional relative coordinates, we need for the perturbations of an inner planet by an outer one the function

$$
R=\mathrm{Gm}_{2} \mathrm{a}_{2}^{-1}\left\{\mathrm{a}_{2} \Delta^{-1}-\mathrm{a}_{2} \mathrm{r}_{1} \mathrm{r}_{2}^{-2} \cos \Phi\right\}
$$

and for the perturbations of an outer planet by an inner one

$$
R=G m_{1} a_{2}^{-1}\left\{a_{2} \Delta^{-1}-a_{2} r_{2} r_{1}^{-2} \cos \Phi\right\}
$$

where $\Delta=\left(r_{1}^{2}+r_{2}^{2}-2 r_{1} r_{2} \cos \Phi\right)^{\frac{1}{2}}$ denotes the mutual distance of the planets, and the expressions in curly brackets are dimensionless. Let us introduce the auxiliary function

$$
\rho=\frac{r_{1} r_{2}}{a_{1} a_{2}} \cos \Phi
$$

then we can write

$-a_{2} r_{1} r_{2}^{-2} \cos \Phi=\frac{a_{1}}{a_{2}} \frac{\partial^{2} \rho}{\partial M_{2}^{2}}$ and $-a_{2} r_{2} r_{1}^{-2} \cos \Phi=\left(\frac{a}{a_{1}}\right)^{2} \frac{\partial^{2} p}{\partial M_{1}^{2}}$

Working with canonical relative coordinates (Poincaré, 1897), we find again $a_{2} \Delta^{-1}$ as the (dimensionless) principal part of the disturbing function. The complementary part, however, turns out to be

$$
-\frac{m_{0} \sqrt{a_{2} / a_{1}}}{\sqrt{m_{0}+m_{1}} \sqrt{m_{0}+m_{2}}} \frac{\partial^{2} p}{\partial M_{1} \partial M_{2}}
$$

where $m_{0}$ stands for the mass of the Sun. This function is the same for the perturbations of the inner and outer planets.

To sum up, the elaboration of a planetary perturbation theory requires in either of these cases the development of the functions $a_{2} \Delta^{-1}$ and 0 into a multiple Fourier series. 
In analogy with equation ( 31 ), the development of the function $a_{2} \Delta^{-1}$ is of the form

$$
a_{2} \Delta^{-I}=\sum_{-\infty}^{\infty} \sum_{-\infty}^{\infty} \sum_{-\infty}^{\infty} \sum_{-\infty}^{\infty} c_{j_{1} j_{2} k \ell} \cos \left[j_{1} M_{1}+j_{2} M_{2}+(k+l) \lambda_{1}-(k-l) \lambda_{2}\right],
$$

whose arguments can also be written as

$$
i_{1} \lambda_{1}+i_{2} \lambda_{2}-j_{1} w_{1}-j_{2} w_{2},
$$

where

$$
i_{1}=j_{1}+k+l \text { and } i_{2}=j_{2}-k+l \text {. }
$$

The coefficients $c_{j_{1} j_{2} k l}$ of the above trigonometric series in turn are power series in the elements $e_{1}, e_{2}$, and $v=\sin ^{2}(\mathrm{~J} / 2)$. Their general expressions by means of Laplace coefficients and Newcomb operators were derived in Special Report No. 140. For the sake of easy reference we repeat here some of the equations used in their computation:

$$
c_{j_{1} j_{2} k l}=c_{-j_{1},-j_{2},-k,-l}=\sum_{s_{1} s_{2}} \sum_{j_{1} j_{2} k l}^{s_{1} s_{2}} e_{1}^{s_{1} e_{2}^{s} 2} \text {, }
$$
the summations being extended over all values of the indices $s_{1}$ and $s_{2}$
for which

$$
s_{1}=\left|j_{1}\right|+2 h_{1}, \quad s_{2}=\left|j_{2}\right|+2 h_{2} \text {, }
$$

and

$$
c_{j_{1} j_{2} k l}^{s_{1} s_{2}}=\prod_{j_{1} j_{2}}^{s_{1} s_{2}}(D \mid k, l) b_{k l}(\alpha) ;
$$

the combined Newcomb operators are defined by

$$
\prod \prod_{j_{1} j_{2}}^{s_{2}}(D \mid k, l)=\prod \prod_{j_{l}}^{s_{l}}(D \mid k+l) \cdot s_{j_{2}}^{s_{2}} \prod(D \mid-k+l) ;
$$


$\alpha=a_{1} / a_{2}, b_{k \ell}(\alpha)=b_{|k \| l|}(\alpha)$, and for $l \geq 0$ the expansion of the Jacobi coefficients in terms of Laplace coefficients is given by

$$
\mathrm{b}_{\mathrm{k} \ell}(\alpha)=\sum_{\mathrm{m}}(-1)^{\mathrm{m}} v^{\ell+\mathrm{m}} \mathrm{b}_{\mathrm{klm}}(\alpha),
$$

where

$$
b_{k \ell m}(\alpha)=\left[\begin{array}{c}
\frac{1}{2} \\
\ell+m
\end{array}\right] \sum_{j=0}^{m}\left(\begin{array}{c}
\ell+m \\
j
\end{array}\right)\left(\begin{array}{c}
\ell+m \\
m-j
\end{array}\right) b_{k+m-2 j}^{\frac{1}{2}+\ell+m} .
$$

In other words, we have

$$
c_{j_{1} j_{2} k l}=\sum_{s_{1} s_{2}} \sum_{s} c_{j_{1} j_{2} k l}^{s_{1} s_{2}^{s}} e_{1}^{s_{1} e_{2}^{s}{ }_{2} s}
$$

with

$$
\begin{gathered}
\mathrm{s}=\ell+\mathrm{m} \text { and } \\
\mathrm{c}_{\mathrm{j}_{I} j_{2} k \ell}^{\mathrm{s}_{I} \mathrm{~s}^{\mathrm{s}}}=(-I)^{\mathrm{m}} \prod_{j_{I} j_{2}}^{\mathrm{s}_{I} \mathrm{~s}_{2}}(\mathrm{D} \mid \mathrm{k}, l) \mathrm{b}_{\mathrm{k} \ell m}(\alpha) .
\end{gathered}
$$

2. The complementary part

There is not much to say about the analytical development of such a simple function as $\rho$. It is easily obtained from the definition of Hansen coefficients by the expansion (32).

The general form

$$
0=\sum_{-\infty}^{\infty} \sum_{-\infty}^{\infty} \sum_{k, l} \gamma_{j_{1} j_{2} k l} \cos \left\lceil j_{1} M_{1}+j_{2} M_{2}+(k+l) \lambda_{1}-(k-l) \lambda_{2}\right]
$$

of this development is the same as $\left(31^{\prime}\right)$. But because in (68)

$$
\begin{gathered}
\cos \Phi=\mu \cos \left(u_{1}-u_{2}\right)+v \cos \left(u_{1}+u_{2}\right), \\
\left(r_{1} / a_{1}\right) \exp i u_{1}=\sum_{-\infty}^{\infty} x_{j_{1}+1}^{1, I} z_{1}^{j_{1}} \zeta_{1} \text { and }\left(r_{2} / a_{2}\right) \exp i u_{2}=\sum_{-\infty}^{\infty} x_{j_{2}+1}^{1,1} z_{2}{ }^{2} \zeta_{2},
\end{gathered}
$$


only the following combinations of the indices $k, l$ occur:

$$
(k, l)=(1,0),(-1,0),(0,1),(0,-1)
$$

The coefficients $\gamma_{j_{1} j_{2} k l}$ are

$$
\begin{aligned}
& Y_{j_{1} j_{2} 10}=Y_{-j_{1},-j_{2},-1,0}=\frac{\mu}{2} x_{j_{1}+1}^{I, I} x_{j_{2}-1}^{I,-1} \\
& Y_{j_{I} j_{2} O I}=Y_{-j_{1},-j_{2}, 0,-1}=\frac{v}{2} x_{j_{1}+1}^{I, I} x_{j_{2}+1}^{I, I} .
\end{aligned}
$$

Thus using the definition

$$
x_{j+k}^{n, k}(e)=\sum_{m} \prod_{j}^{m}(n \mid k) e^{m}
$$

of the Newcomb "operators," we find the expansions

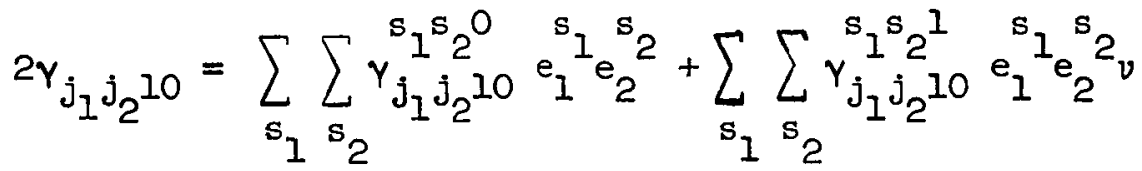

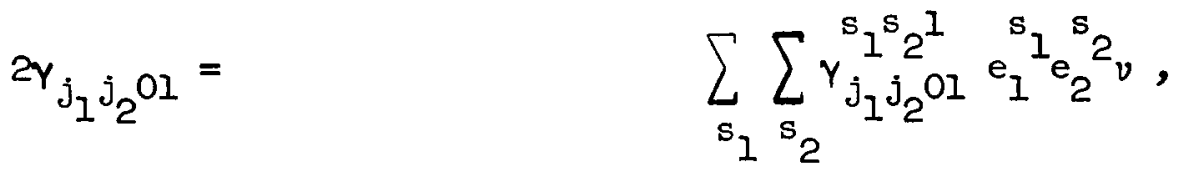

where

$$
\begin{aligned}
& \frac{r_{1} s_{2} 0}{y_{1} j_{2} 10}=-\gamma_{j_{1} j_{2}}^{s_{10} s_{2}}=\prod_{j_{1}}^{s_{1}}(I \mid I) \prod_{j_{2}}^{s_{2}}(I \mid-I) \\
& r_{j_{1} j_{2} 01}^{s_{1} 2^{I}}=\prod_{j_{1}}^{s}(I \mid I) \prod_{j_{2}}^{s_{2}}(I \mid I) .
\end{aligned}
$$

Then the coefficients in the Fourier series of the (dimensionless) complementary part of the disturbing function are 


$$
-\alpha i_{2} i_{2} \gamma_{j_{1} j_{2} k l} \text { and }-\alpha^{-2} i_{1} i_{1} \gamma_{j_{1} j_{2} k l}
$$

for conventional relative coordinates,

and

$$
\frac{m_{0}^{\alpha-\frac{1}{2}}}{\sqrt{m_{0}+m_{1}} \sqrt{m_{0}+m_{2}}} i_{1} i_{2} \gamma_{j_{1} j_{2} k l}
$$

for canonical relative coordinates.

From now on we assume that the principal and complementary parts of the disturbing function have been merged. That is, the c-coefficients are supposed to have been modified so as to include the contribution of the $\gamma$-coefficients.

\section{The pattern of indices}

Once the mathematics of the developments $\left(31^{\prime}\right)$ and (75) is formulated and the several required subroutines are all coded, our problem consists of organizing the latter into an efficient unity.

The ultimate building elements of the disturbing function depend on seven indices. Each of these indices $j_{1}, j_{2}, k, l, s_{1}, s_{2}, s$ has a theoretically infinite range. In practical applications, of course, the relevant expansions have to be limited to a finite, although sometimes a very large number of terms. Various circumstances can be used with advantage.

The coefficients $c_{j} j_{2} k l$ of our quadruple Fourier series contain the factor $e_{1}^{\left|j_{1}\right|} e_{2}^{\left|j_{2}\right|} v \mid l^{j_{1}}{ }^{j_{2} k \ell}$ As $e_{1}, e_{2}$, and $v^{\frac{1}{2}}$ are small quantities of usually comparable order of magnitude, it is natural to limit the development to those values of the indices, $j_{1}, j_{2}$, and $\ell$, for which

$$
p=\left|j_{1}\right|+\left|j_{2}\right|+2|\ell|,
$$

called the rank of a term, does not exceed a certain preassigned integer $P$. Similarly, with the expansion (69) we associate the integers

$$
q=s_{1}+s_{2}+2 s s,
$$

and call them the degree of a coefficient $c_{j_{1} j_{2} k l}^{s_{1} s_{2} s}$. Note that $q-p=2 r$ is always a nonnegative even integer. Needless to say, the proper limits $P$ and $Q$ depend largely on the pair of planets under consideration. 
- In general, the Laplace coefficients and their derivatives decrease with increasing absolute values of the index $\mathrm{k}$. Moreover, the integration of the Fouriex series derived from (3I') introduces divisors that, as a rule, slowly increase at the same time. (Long-period terms with small divisors constitute a notable exception to this rule. Like the secular terms, they must be treated with special care.) Thus we may also impose a condition $|\mathrm{k}| \mathrm{s}$.

Due to the symmetry in relation $\left(30^{\prime}\right)$, it is sufficient to compute the terms of the disturbing function the indices of which are

$$
\begin{array}{lllll}
j_{1}=0 & j_{2}=0 & l=0 & (p=0) & \mathrm{k}=0 \\
j_{1}=0 & j_{2}=0 & l=0 & (\mathrm{p}=0) & \mathrm{k}=1, \ldots, \mathrm{K} \\
j_{1}>0 & j_{2}=0 & l=0 & \left(\mathrm{p}=j_{I}=0, \ldots, P\right) & \mathrm{k}=-\mathrm{K}, \ldots, \mathrm{K}=-\mathrm{K}, \ldots, \mathrm{K} \\
& j_{2}>0 & l=0 & \left(\mathrm{p}=\left|\mathrm{j}_{1}\right|+\mathrm{j}_{2}=0, \ldots \mathrm{P}\right) & \left(\mathrm{p}=\left|\mathrm{j}_{1}\right|+\left|\mathrm{j}_{2}\right|+2 l=0, \ldots, P\right) \mathrm{k}=-\mathrm{K}, \ldots, \mathrm{K}
\end{array}
$$

If the terms belonging to these indices are known, then the complete expression ( $3 I^{\prime}$ ) in the range $\mathrm{p} \leq \mathrm{P},|\mathrm{k}| \leq \mathrm{K}$ is obtained by taking twice all the terms just specified, except the first one, which is a constant.

Let us give a concrete example. The 98 solutions of the inequality $\left|j_{1}\right|+\left|j_{2}\right|+2 \ell \leq 6$ in question are:

$\begin{array}{rrrrrrrrrrrr}j_{1} & j_{2} & l & j_{1} & j_{2} & l & j_{1} & j_{2} & l & j_{1} & j_{2} & l \\ 0 & 0 & 0 & 0 & 4 & 0 & 0 & 3 & 1 & 4 & 0 & 1 \\ & & & -1 & 3 & 0 & -1 & 2 & 1 & 3 & 1 & 1 \\ 1 & 0 & 0 & -2 & 2 & 0 & -2 & 1 & 1 & 2 & 2 & 1 \\ 0 & 1 & 0 & -3 & 1 & 0 & -3 & 0 & 1 & 1 & 3 & 1 \\ & & & 2 & 0 & 1 & -2 & -1 & 1 & 0 & 4 & 1 \\ 2 & 0 & 0 & 1 & 1 & 1 & -1 & -2 & 1 & -1 & 3 & 1 \\ 1 & 1 & 0 & 0 & 2 & 1 & 0 & -3 & 1 & -2 & 2 & 1 \\ 0 & 2 & 0 & -1 & 1 & 1 & 1 & -2 & 1 & -3 & 1 & 1 \\ -1 & 1 & 0 & -2 & 0 & 1 & 2 & -1 & 1 & -4 & 0 & 1 \\ 0 & 0 & 1 & -1 & -1 & 1 & 1 & 0 & 2 & -3 & -1 & 1 \\ & & & 0 & -2 & 1 & 0 & 1 & 2 & -2 & -2 & 1 \\ 3 & 0 & 0 & 1 & -1 & 1 & -1 & 0 & 2 & -1 & -3 & 1 \\ 2 & 1 & 0 & 0 & 0 & 2 & 0 & -1 & 2 & 0 & -4 & 1 \\ 1 & 2 & 0 & & & & & & & 1 & -3 & 1 \\ 0 & 3 & 0 & 5 & 0 & 0 & 6 & 0 & 0 & 2 & -2 & 1 \\ -1 & 2 & 0 & 4 & 1 & 0 & 5 & 1 & 0 & 3 & -1 & 1 \\ -2 & 1 & 0 & 3 & 2 & 0 & 4 & 2 & 0 & 2 & 0 & 2 \\ 1 & 0 & 1 & 2 & 3 & 0 & 3 & 3 & 0 & 1 & 1 & 2 \\ 0 & 1 & 1 & 1 & 4 & 0 & 2 & 4 & 0 & 0 & 2 & 2 \\ -1 & 0 & 1 & 0 & 5 & 0 & 1 & 5 & 0 & -1 & 1 & 2 \\ 0 & -1 & 1 & -1 & 4 & 0 & 0 & 6 & 0 & -2 & 0 & 2 \\ & & & -2 & 3 & 0 & -1 & 5 & 0 & -1 & -1 & 2 \\ 4 & 0 & 0 & -3 & 2 & 0 & -2 & 4 & 0 & 0 & -2 & 2 \\ 3 & 1 & 0 & -4 & 1 & 0 & -3 & 3 & 0 & 1 & -1 & 2 \\ 2 & 2 & 0 & 3 & 0 & 1 & -4 & 2 & 0 & 0 & 0 & 3 \\ 1 & 3 & 0 & 2 & 1 & 1 & -5 & 1 & 0 & & & \\ & & & 1 & 2 & 1 & & & & & & \end{array}$


To find the appropriate indices $s_{1}, s_{2}$, s we recall that

$$
s_{1}=\left|j_{1}\right|+2 h_{1}, \quad s_{2}=\left|j_{2}\right|+2 h_{2}, \quad \text { and } \quad s=l+m
$$

so that

$$
h_{1}+h_{2}+m=r \leq \frac{1}{2}(Q-p)
$$

For example, corresponding to the choice $P=6$ and $Q=8$, we get:

$$
\begin{aligned}
& \begin{array}{llll}
p=0 & p=1,2 & p=3,4 & p=5,6 \\
r=4 & r=3 & r=2 & r=1
\end{array}
\end{aligned}
$$

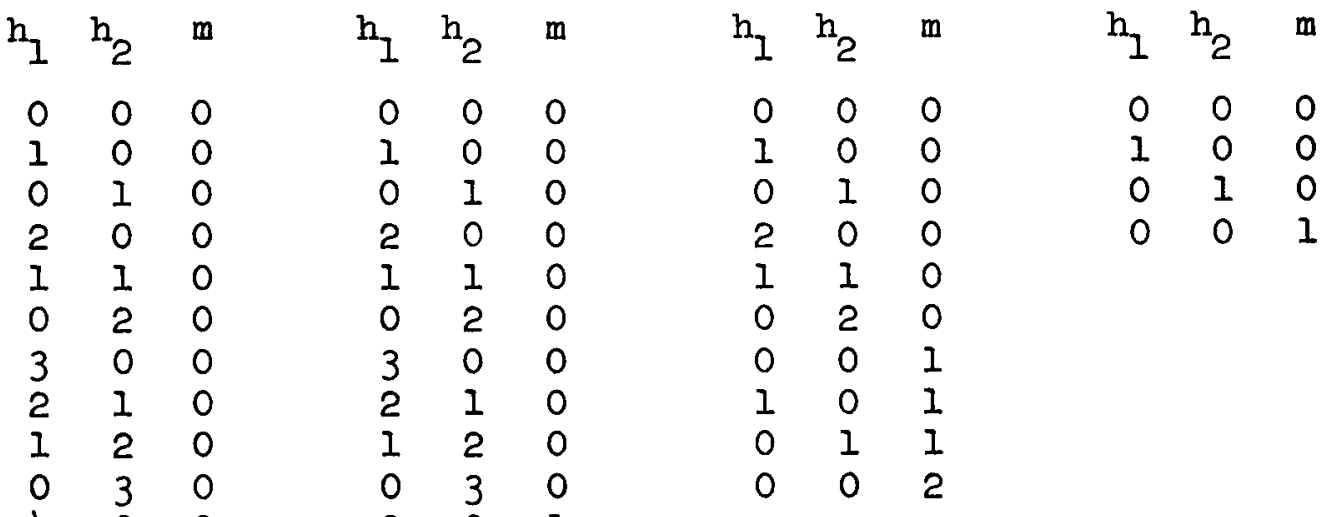

$$
\begin{aligned}
& \begin{array}{lll}
0 & 3 & 0 \\
4 & 0 & 0
\end{array} \\
& \begin{array}{llllll}
3 & 1 & 0 & 1 & 0 & 1
\end{array} \\
& 2 \quad 2 \quad 0 \quad 001101 \\
& \begin{array}{llllll}
1 & 3 & 0 & 2 & 0 & 1 \\
0 & 4 & 0 & 1 & 1 & 1
\end{array} \\
& \begin{array}{llllll}
0 & 0 & 1 & 0 & 2 & 1
\end{array} \\
& \begin{array}{llllll}
1 & 0 & 1 & 0 & 0 & 2
\end{array} \\
& \begin{array}{llllll}
0 & 1 & 1 & 1 & 0 & 2
\end{array} \\
& \begin{array}{llllll}
2 & 0 & 1 & 0 & 1 & 2 \\
1 & 1 & 1 & 0 & 0 & 3
\end{array} \\
& \begin{array}{lll}
1 & 1 \\
0 & 2 & 1
\end{array} \\
& \begin{array}{lll}
3 & 0 & 1 \\
2 & 1 & 1
\end{array} \\
& \begin{array}{lll}
1 & 2 & 1
\end{array} \\
& \begin{array}{lll}
0 & 3 & 1 \\
0 & 0 & 2
\end{array} \\
& \begin{array}{lll}
1 & 0 & 2
\end{array} \\
& 012 \\
& 202 \\
& \begin{array}{lll}
1 & 1 & 2
\end{array} \\
& \begin{array}{lll}
1 & 2 & 2
\end{array} \\
& 003 \\
& 103 \\
& \begin{array}{lll}
0 & 1 & 3 \\
0 & 0 & 4
\end{array}
\end{aligned}
$$


- The size of the individual coefficients is in reality much less regular than the foregoing general remarks would seem to indicate. Human judgment as to which terms should be kept and which neglected is here probably preferable to computer-made decisions. The efficient use of the present program is facilitated by the following features. First, for each combination of the $j_{1}, j_{2}, l$ indices we specify separately the degree $q$ of the coefficients to be retained, as well as an interval $k \leq k \leq \bar{k}$ of the Index $\mathrm{k}$. Second, the program can be run in two modes, namely either to com-

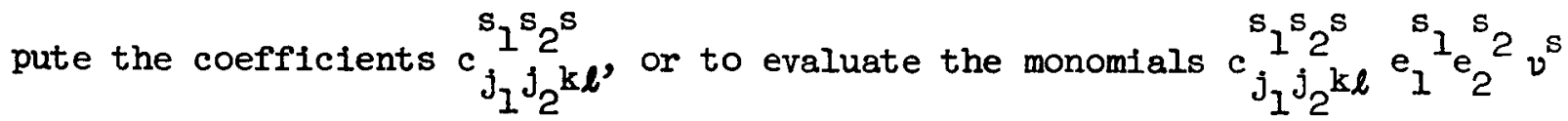
This way we get a fair idea about the convergence of the analytical development of the disturbing function in any particular case. Third, the effect of the divisors of integration is also exhibited. We shall say more about the program in Part 4.

Since the quantities $n_{1}, n_{2}$ are the mean motions of the two planets, the angular frequency of a term in the Fourier series (3I') is approximately ${ }^{1} n_{1}+1_{2} n_{2}$. It is therefore of interest to know which indices $j_{1}, j_{2}, k$, $l$ can give rise to a certain combination

$$
1_{1}=j_{1}+k+l \text { and } 1_{2}=j_{2}-k+l
$$

These relations are equivalent to

$$
\begin{aligned}
& j_{1}+j_{2}+\varepsilon l=1_{1}+i_{2}, \\
& j_{1}-j_{2}+2 k=1_{1}-i_{2} .
\end{aligned}
$$

For a given pair of indices $i_{1}, i_{2}$ equation (76) has an infinite number of solutions. But we are interested only in solutions with rank $p \leq P$, and these are finite in number. Indeed, the indices $j_{1}, j_{2}, l$ satisfying the conditions (76) and $\left|j_{1}\right|+\left|j_{2}\right|+2|\ell| \leq P$ are:

$$
\begin{aligned}
\ell & =-[\mathrm{v} / 2], \ldots,[\mathrm{u} / 2]-1,[\mathrm{u} / 2], \\
\left(j_{1}, j_{2}\right) & =([\mathrm{u}]-2 \ell,-[\mathrm{v}]), \ldots,(-[\mathrm{v}],[\mathrm{u}]-2 \ell) \quad \text { if } \ell \geq 0, \\
\left(j_{1}, j_{2}\right) & =([\mathrm{u}],-[\mathrm{v}]-2 \ell), \ldots,(-[\mathrm{v}]-2 \ell,[\mathrm{u}]) \quad \text { if } \ell<0,
\end{aligned}
$$

with

$$
u=\frac{1}{2}\left(P+i_{1}+i_{2}\right) \geq 0 \text { and } \quad v=\frac{1}{2}\left(P-i_{1}-i_{2}\right) \geq 0
$$


Once a pair $\left(j_{1}, j_{2}\right)$ has properly been chosen, the value of the index $k$ is determined by equation (77). It is sufficient to obtain the quadruples $\left(j_{1}, j_{2}, k, l\right)$ under the condition $i_{1}+i_{2} \geq 0$; whenever the convention adopted on p. 7 is violated, we simply change signs, except in the case $i_{1}+i_{2}=0$, where the quadruples violating our convention can be disregarded.

As an example, let us consider the great inequality in the theories of Jupiter and Saturn associated with the arguments

$$
-2 \lambda_{1}+5 \lambda_{2}-j_{1} w_{1}-j_{2} w_{2}
$$

Due to their small divisors, such terms of the disturbing function must be carried up to an exceptionally high rank. Put $P=9$, so that $u=6, v=3$. The appropriate solutions of equation (76) are exhibited in the following figure, where open circles indicate that the sign of the corresponding indices is to be changed.

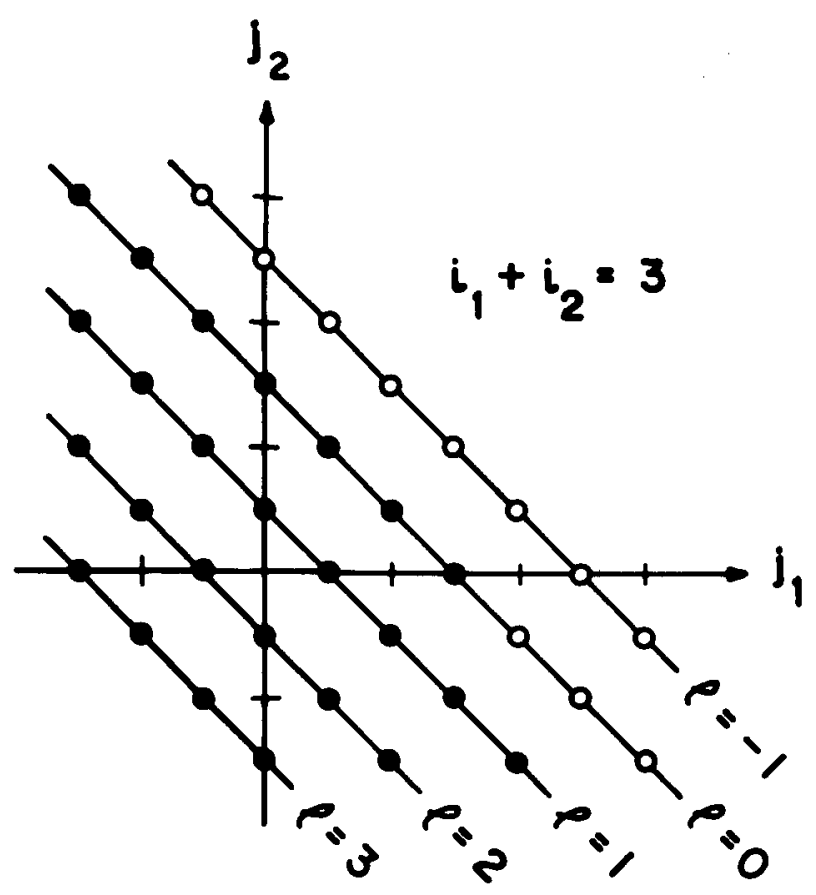

\section{The program}

\section{Introduction}

The program is written in FORTRAN II for the IBM 7094 computer and double-precision arithmetic is used. It computes the coefficients

${ }_{j_{1} j_{2} k l}^{s_{1}{ }^{s}}(70)$ of the Fourier expansion of the disturbing function by applying the combined Newcomb operators (see Special Report No. 140) to the Jacobi coeffi- 
clents which are derived from the Laplace coefficients (see Special Report No.129). It also gives the flrst and second derivatives $\mathrm{DC}$ and $\mathrm{D}^{2} \mathrm{C}$, where $\mathrm{D}=\alpha \frac{\partial}{\partial_{\alpha}}$ and $C=c_{j_{1} j_{2} k l}^{s_{1} s_{2} s}$, and the fractions $C / N, D C / N$, and $C / N^{2}$, where $N=i_{1}+\beta i_{2}$.

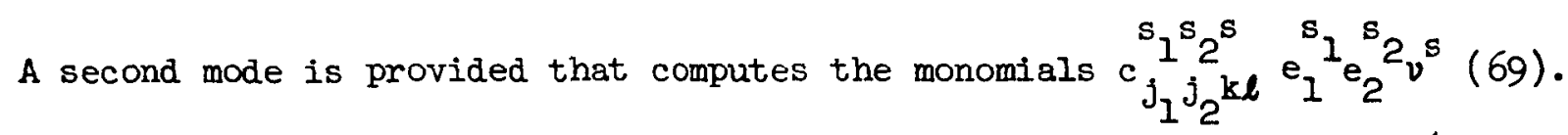
The complementary parts are computed in conventional relative coordinates (inner or outer planets), or in canonical relative coordinates as specified by the user.

\section{Program limitations}

There are two versions of the program. The first and smaller version requires 13356 cells of lower core and 3341 cells of common storage. The limitations of this version are:

$$
\begin{aligned}
& |\mathrm{k}| \leq 15, \ell \geq 0 \quad \text { for } \mathrm{k} \text { and } \ell \text { in } c_{j_{1} j_{2} \mathrm{k} \ell}^{s_{2}}, \\
& \text { the degree } \mathrm{q} \leq 8, \\
& \text { the rank } \mathrm{p} \leq 6 .
\end{aligned}
$$

The second version requires 15360 cells of lower core and 6305 cells of common storage. Here the limitations are:

$$
|k| \leq 20, \quad l \geq 0, q \leq 10, \text { and } p \leq 9
$$

\section{Method}

First the Laplace coefficients $D^{j_{b} s+\frac{1}{2}}$ are computed and stored for later use. Next we generate the inner and outer Newcomb operators and store the coefficients of these polynomials. The program then reads in the parameters $j_{1}, j_{2}, l$, the minimum and the maximum limits of $k$, and the degree $q$ of the coefficients $c_{j_{1} j_{2} s_{l}{ }^{s}}^{s_{l}}$ we want to compute.

All possible combinations of $s_{1}, s_{2}$, and $s$ for the given parameters $j_{1}$, $j_{2}, l$, and $q$ are computed and stored. Then for each $k$ from $\mathrm{k}_{\min }$ to $\mathrm{k}_{\max }$ and for each combination of $s_{1}, s_{2}$, and $s$ we recall from storage the corresponding Inner and outer Newcomb operators $\Pi \prod_{j_{1}}^{s_{1}}$ and ${ }_{j_{2}}^{s_{2}} \Pi$ and evaluate them for the current values of $(k+l)$ and $(-k+l)$, respectively $\left(26^{\prime}\right)$. The two resulting polynomials in the differential operator $D$ are multiplied together. The product polynomial is the combined operator $\prod_{j_{1} j_{2}}^{s_{1} s_{2}}\left(26^{\prime}\right)$. Next the $b_{k l m}$ and all its required derivatives are determined for each new $m$. Finally we apply the combined operator to the $b_{k l m}(70)$. 
If the $c_{j_{1} j_{2} k l}^{s_{1}{ }^{s}}$ have to be evaluated for a very large number of

indices, it is more efficient to compute all the possible combinations of the $b_{k \ell m}$ directly after the computation of the Laplace coefficients, storing them for later use. In the smaller version of the program this would require an extra 1200 locations.

Because of the symmetry $c_{j_{1} j_{2} k \ell}=c_{-j_{1}},-j_{2},-k,-l\left(30^{\prime}\right)$ we evaluate only half of the coefficients and then multiply each of them by two except the $c_{0000^{-}}$To avoid duplication, we adopt the convention that $l$ is always greater than or equal to zero and if $l=0$ then $j_{2} \geq 0$. If this convention is violated we simply reverse the signs of all four indices.

If $|\mathrm{k}|+\ell=1$ and the coefficients of the complementary parts in formula (75) are nonzero then we compute the complementary parts of the c-coefficients in the coordinates specified by the user in the input and add them to the principal parts. In these cases the printout is preceded by an asterisk.

Usage

The first data card should contain six control characters for the printing of intermediate results using the format (6II0). They control the printing of the Laplace coefficients, the inner and the outer J's, the inner and the outer $X ' s$ (Special Report No. 140), and the time used by the program, in that order. A blank card will suppress the printing of all intermediate results.

Each group of input cards with indices must be preceded by a control card which indicates the mode and the choice of coordinates and gives the values of the program parameters. The first character on a control card should be either $a^{\prime} C$ ' for coefficients or a ' $M$ ' for monomials followed by a 1 , 2 , or 3 for conventional relative coordinates, inner or outer planet, or canonical relative coordinates, respectively. The values for $\alpha\left(0<\alpha=a_{1} / a_{2}<1\right)$ and $\beta\left(0<\beta=n_{2} / n_{1}<1\right)$ follow, and if monomials are requested, the values for $e_{1}, e_{2}$, and $\sqrt{v}(=\sin \mathrm{J} / 2)$. Finally, if canonical relative coordinates were specified, $m_{0} / m_{1}$ and $m_{0} / m_{2}$. The control cards are in free-field format, and numerals are defined by a tralling blank. 
This control card is followed by a group of parameter cards, each card containing $j_{1}, j_{2}, l, \mathrm{k}_{\min }, \mathrm{k}_{\max }$, and $\mathrm{q}$ with a format of (6IIO). For each $j_{1}, j_{2}, k$, and $l$ where $k$ runs through all the integral values from $k_{\min }$ to $k_{\max }$, all the combinations of $s_{1}, s_{2}$ and $s$ such that $s_{1}+s_{2}+2 s s q$ are generated. For every set of indices $s_{1}, s_{2}, s_{1} j_{1}, j_{2}, k, l$, the coefficient $c_{j_{1} j_{2} k \ell}^{s_{2} s}$ its first two Newcomb derivatives, and the fractions $C / N, D C / N$, and $\mathrm{C} / \mathrm{N}^{2}$ are evaluated and printed out. If the value for $\mathrm{q}$ is preceded by a minus sign on the input card, the printout will be suppressed.

To end a group of parameter cards a blank card is inserted. The program then reads a new control card for the next group of parameter cards. In order to terminate the program a card with a zero or a decimal point is placed directly after the blank card.

We shall now discuss the MAIN program and the subroutines that have not already been described in the Special Reports Nos. 129 and 140.

\section{MAIN program}

After the necessary initializations and the reading of the print-control card, the MAIN program reads the first control card into a buffer. The program will exit if the coordinate code number is zero. Hence a card with a zero or a decimal point in the place of a control card will terminate the program. A blank card may not be used since the numerals on the control card are read in a free-field format. If the coordinate code number is not zero, the first character of the control card image is read from the buffer. If this character is neither a ' $\mathrm{M}$ ' or a ' $\mathrm{C}$ ' an error message is returned and the program exits. If it is $a{ }^{\prime} C$ ' the card is read for the second time from the buffer and $\alpha, \beta, m_{1}$, and $m_{2}$ are picked up. For monomials, $1 . e .$, in case of a ' $M$ ', $\alpha, \beta, e_{1}, e_{2}, \sqrt{v}, m_{1}$, and $m_{2}$ are read and the coordinate control code is set negative. The program then calls the subroutine MORCOE to compute and store the Laplace coefficients. If the first print control is greater than zero, the Laplace coefficients are printed out. Next the subroutine COMBOP is called to develop the inner and outer Newcomb operators and store them in two linear arrays. The appropriate headings are written according to the choice of coordinates. If the coordinate code is negative, i.e., if monomials were requested, the values of $e_{1}, e_{2}$, and $v$ are printed out and their powers are computed and stored.

The program now proceeds to read a parameter card with $j_{l}, j_{2}, l, k_{\text {min }}$, $\mathrm{k}_{\max }$, and $\mathrm{q}$ using the format (6IIO). Compliance with the program limitations is checked, and if not satisfied an error message is returned. 
The subroutine INDEX finds and stores all possible combinations of $s_{1}, s_{2}$, and $s$ for the given set of parameters. The program ends with a loop in which $k$ is stepped up from $k_{\min }$ to $k_{\max }$. For each $k$ and each combination of $s_{1}, s_{2}$, and $s$ the subroutine SOLVE is called to compute and print the ${ }^{c_{1} s_{2} s^{1}}{ }_{j_{1} j_{2} k l}$

The program then goes back and reads the next parameter card. If a blank parameter card is encountered, the page line count is reset and a new control card is read in. If the new $\alpha$ differs from the previous one, the Laplace coefficients are computed again for the new $\alpha$.

\section{MORCOE}

The subroutine MORCOE first computes the Laplace coefficients for $s+\frac{1}{2}=\frac{1}{2}$ by calling LPLCOF. This program LPLCOF is described in detail in Special Report No. 129 and is used here essentially unchanged except for dimensions and storage arrangement.

All the results of LPLCOF are stored in the common area and are later overwritten by the results of the Newcomb operator program. MORCOE then computes the Laplace coefficients $D^{j} b_{k}^{s+\frac{1}{2}}$ from $s+\frac{1}{2}=1 \frac{1}{2}$ to $s+\frac{1}{2}=4 \frac{1}{2}$ by recursion and stores the results in lower core for later use by the BKLM program.

Using formula (16) in Special Report No. 129 we compute $D^{j_{b}} b_{k}^{s+\frac{1}{2}}$ for $s=1,2,3,4$, and $j=0, \ldots, Q-2 s+2$, first for $k=0$ (taking $D^{j_{b} s+\frac{1}{2}}=D^{j_{b} s+\frac{1}{2}}$ ) and then for $k=1, \ldots, 19$ (smaller version). COMBOP

The subroutine COMBOP constructs the inner and outer Newcomb operators and stores their coefficients for later use in two linear arrays AICM and ATEMP, respectively.

It does so by calling the Newcomb operator program written at M.I.T. and described in the second part of Special Report No. 140. We have adapted this program to fit our needs. We were mainly concerned with storage Iimitations and made some changes of which the more important are the following. The numbers in column 16 of array AICT on the bottom of page 30 (Special Report. No. 140) were eliminated. This made it possible to store the coefficients of all inner and outer operators up to degree 8 in two arrays AICM and ATEMP each of only 625 double words. These two arrays occupy the same space in the common area that was used previously for all the intermediate results of the Laplace coefficients program. The PRINT routine was 
simplified, and INTVAL was eliminated. The routines GETAS, GETPSI, and BIGPSI are not needed for the present application. We added the routine OUIRJS which constructs the outer J's from the inner J's by substituting (-D-I) for D (page 14, Special Report No. 140) which is accomplished by the subroutine NSUBST. The outer J's are stored in ATEMP. The subroutines STORE and KEEP are combined to one routine and modified so that it now can service both arrays AICM and ATEMP. The subroutines TRANS and DIVIDE are similarly changed.

After calling GETJS, COMBOP calls OUTRJS and then twice GETXS, once with AICM as argument and the second time with ATEMP to produce the inner and outer $\mathrm{X}^{\prime} \mathrm{s}$. The X-arrays overwrite the J-arrays in storage.

Having generated and stored the Laplace coefficients and the inner and outer Newcomb operators, we are ready to enter the inner loop of the program which computes and prints the $c_{j_{1} j_{2} k l}^{s_{1} s_{2}}$ for each set of indices. OUTRJS

OUTRJS moves each inner $J$ to the working array AICT with the subroutine TRANS, then converts it with NSUBST to the corresponding outer $J$ and stores this outer $J$ in the array ATEMP.

NSUBST

The subroutine NSUBST constructs each element of the outer J-array by multiplying each element of the same or higher degree in D in the same kcolumn of the inner J-array by the proper coefficient of the Pascal triangle, which triangle was stored during the development of the Newcomb operators, and by the proper alternating sign and then adding these products together.

INDEX

The subroutine INDEX generates all combinations of $s_{1}$, $s_{2}$, and $s$ according to the scheme on page 8 and stores the resulting sets in a twodimensional array. The routine consists of three loops. The total number of combinations $s_{1}$, $s_{2}$, and $s$ for each set of indices is also returned to SOLVE

The subroutine SOLVE has two functions, the computation and the printing of the coefficients $c_{j_{1} j_{2} k l^{s}}^{s_{1}}$ 
The format for the headings is constructed during execution time. The spacing of the coefficients of the cosine argument is handled by a subroutine FORMAT. If the divisor $N=i_{1}+\beta i_{2}$ is zero, only the first half of the heading is printed and three columns instead of six are written out.

If $|\mathrm{k}|+\ell=I$ and the coefficients of the $\gamma$-components (75) belonging to the selected coordinate system are nonzero, then there will be a conplementary part. The format is adjusted so that the heading is preceded by an asterisk and a flag is set for the computation of the complementary part. A running line count is kept lest the headings are split over two pages.

After the headings are written out, the coefficients are computed in a loop that is repeated for each combination of $s_{1}, s_{2}$, and $s$. The first time through the loop and each time $m$ changes, the $b_{k l m}$ and its derivatives are evaluated by the subroutine BKLM from the Laplace coefficients. The appropriate combined Newcomb operator now is produced by the subroutine COMBIN in the form of a polynomial in D. The subroutine APPLY then applies this combined operator to the proper derivatives of $\mathrm{b}_{\mathrm{k} \ell \mathrm{m}}$ to yield the $c_{j_{1} j_{2} k l}^{s_{1} j^{s}}(70)$, the $D_{c_{j_{1}} j_{2} k l}^{s_{2}}$, and the $D^{2} c_{j_{1} j_{2} k l}^{s_{1} s_{2}}$. Because of the symmetry in $\left(30^{\prime}\right)$ the coefficients are doubled except for the $c_{0000}{ }^{1} 2^{5}$ and then divided by $\mathrm{N}$ and $\mathrm{N}^{2}$.

If the flag for a complementary part is set, the value of $s$ is checked. If $s$ is greater than one, the flag is erased. But if $s=0$ or $s=1$, the function program GAMMA computes $\pm \prod_{j_{1}}^{s_{1}}(1 \mid 1) \prod_{j_{2}}^{s_{2}}(1 \mid \pm 1)$ and depending on which coordinate-system was selected, $\gamma$ then is multiplied by the relevant factor in (75). The complementary part and its first and second derivatives are added to the corresponding principal parts. In case of monomials all coefficients and quotients are multiplied by $\mathrm{e}_{1}{ }_{1} e_{2}{ }_{2}{ }_{v}^{s}$.

After the final loop is satisfied, control is returned to the MAIN program to pick up a new set of indices.

\section{FORMAT}

This function subprogram checks each coefficient in the cosine argument. If it is positive $a+s i g n$ is inserted in the format. Proper spacing is provided depending on whether the integers have one or two digits.

$\underline{\text { BKLM }}$

Using the binomial coefficients computed by the Newcomb operator program and the Laplace coefficlents computed by MORCOE, the terms of the sum in (12) are evaluated and summed. The sum is then multiplied by $\left[l^{\frac{1}{2}}\right]$ and given the 
sign from formula (70). This is repeated for all derivatives up to degree $(q-2 s+2)$.

COMBIN

This subroutine moves the inner Newcomb operator $\left.T\right|_{j_{1}} ^{s_{1}}$

from the linear array AICM to the working array AICT. If $j_{I}$ happens to be negative, we change first the signs of $j_{1}, k$, and $l$, using the equality (33), since the Newcomb operators were constructed only for $\rho \geq \sigma$ in view of the symmetry relation (33) or (37) of Special Report No. 140. It then calls the subroutine EVALUK which evaluates this polynomial by substituting for $\mathrm{k}$ the current numerical value of $\mathrm{k}+\ell$. The same procedure is followed for the outer operator $\left.\begin{array}{c}\mathrm{s}_{2} \\ j_{2}\end{array}\right\rceil$ except that this time the polynomial is evaluated for the value $(-\mathrm{k}+\mathrm{l})$.

The subroutine MPPOLY multiplies the two polynomials in D and stores the resulting combined operator. Control then is returned to the SOLVE program.

EVALUK

Subroutine EVALUK simply multiplies each element of the array AICT by the proper power of the constant $k+l$ for the inner operators and $-k+l$ for the outer operators, and sums the products in each row.

MPPOLY

MPPOLY multiplies the two polynomials that resulted from the two calls to EVALUK. It first establishes the degree of the product polynomial. Then each term of the product polynomial is assembled by forming all products that contribute to this term and summing these products. They are found by stepping up the degree of the term in the first polynomial and stepping down the degree in the second polynomial, thus keeping the sum of the degrees constant and equal to the degree of the term of the product polynomial being assembled.

\section{$\underline{\text { APPLY }}$}

The subroutine APPLY multiplies each numerical coefficient of the combined operator with the corresponding derivative of $b_{k l m}$ and with the first and second higher derivatives. Each of these products is summed into its

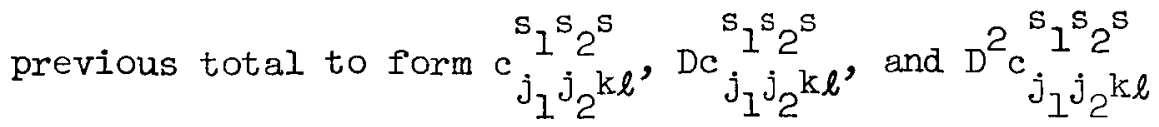


To compute the $\gamma^{\prime}$ s of formula (74) for the complementary part, we first check the value of $k$. If $k$ is nonzero, $|k|$ must be one and $\ell$ must be zero. Otherwise an error message is returned. If $k$ is negative we change the signs of $j_{1}, j_{2}$, and $k$. Then we evaluate $\prod_{j_{1}}^{s_{1}}(I \mid I)$ and $\prod_{j_{2}}^{s_{2}}(I \mid-1)$ with the function routine PI and multiply them together. If $s=0$, we return. If $s=1$, we change sign. In case $k=0$, we check if $\ell=I$ and $s=I$. If this is true, we compute $\gamma=\prod_{j_{1}}^{s}(I \mid I) \prod_{j_{2}}^{s}(I \mid I)$. All other cases result in an error message and exit from the program.

$\underline{P I}$

PI evaluates $\prod_{j_{1}}^{s_{1}}(I \mid I)$ and $\prod_{j_{2}}^{s_{2}}(I \mid-I)$. If a $j$ is negative, we change the sign of $j$ and of $k$, using the relation (33). Then with equation $\left(36^{\prime}\right)$ we find $\prod_{j}^{s}$ from the inner $x$-array and move it to AICT with the routine TRANS.

We add all elements of each column and then add these column totals, if $\mathrm{k}=1$. Or if $\mathrm{k}=-1$, we form an alternating sum.

Immediately following the references, we give two print-out samples of the program. They both pertain to the planets Jupiter and Saturn. Appendix I presents (in mode C) a somewhat more extended development of the disturbing function than Leverrier's classical one in the Annales de l'Observatoire de Paris, vol. 10, p. 68 and pp. 72-93. It was produced by the smaller version of our program; the required machine-time was about one minute. Appendix II exhibits (in mode $M$ ) the secular terms and the long-period terms of the great inequality up to the 9 th powers of the eccentricities, whenever necessary to conform with modern standards of accuracy. 
CHARLIER, C. L.

1927. Die Mechanik des Himmels, 2nd ed., Vol. I, W. de Gruyter \& Co., Leipzig, pp. 228-269.

IZSAK, I. G., and BENIMA, B.

1963. Laplace coefficients and their Newcomb derivatives. Smithsonian Astrophys. Obs. Special Report No. 129.

IZSAK, I. G., GERARD, J. M., EFTMBA, R., and BARNETT, M. P.

1964. Construction of Newcomb operators on a digital computer. Smithsonian Astrophys. Obs. Special Report No. 140.

POINCARE, H.

1897. Sur une forme nouvelle des equations du problème des trois corps. Bull. Astron., Vol. 14, pp. 53-67. 
Appendix I 


\section{THIS \\ COMPUTER RUN \\ IS DEDICATED \\ TO THE MEMORY OF \\ U.-J. LE VERRIER}

\section{(1811-1877)}


$\cos 1 \cdot 0 a_{1}+a_{2}+a y_{1}+0 w_{2}$

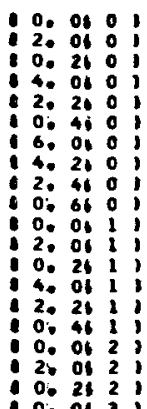

\subsection{E 00} 2.173033eE-01 2.1730330E-01 1.1996160E-01 $1.1556209 E$ OO
$5.0650056 \mathrm{E}-01$ $5.8650056 E-01$
$9.4474330 E-02$ 2.357as27E 00 $1.2371467 E$ OO $1.6852656 E$ OO $1.6852656 E$
$-\$ .6921352 E-01$ $-4.6224638 E$ OO $-4.6224 a 38 E$ OO $-9.4314108 E$ 00 $-6.4137527 E$ OI $-2.4940595 \mathrm{E}$ OI 4.1870770 OO - 1026285e ol i.1820285E O1 DC 2.2065981E-02 $7.8442622 E=0$ $7.8442622 E=0$ $9.3373426 E-01$ $7.1807815 E$ OO $3.0400696 E$ OO 1.1400470 0 $2.453373 E$ oI 1.4050127e ol -3.1377049e od -2.8723126e OL $-2.8723126 E$ o1 -9.8135091e ol -6.06005TEE 02 $-6.0600578 E 02$ -2.1922936 02 5.9164422E 02 5.9164422802 S.9164422E 02
$-2.4485014 E$
02

1806011.0

$\cos 1+1 \mathrm{~L}_{1}-\mathrm{IL}_{2}+\mathrm{OH}+\mathrm{OW} \mathrm{w}_{2}$

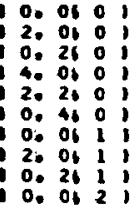
$c$ $742 E-02$ $7.5361742 E-02$ $2.4643393 E-01$ 2.\$643393E-01 1.6728477E 0O $8.3955046 \mathrm{E}-01$ $-1.2118809 E$ OO $-7.4304928 E$ OO $-7.4306928 E$
$7.7525085 E$

OC
$2.636867 E-01$
$1.1508004 E$ OO
$1.150804 E$ OO
$1.3395223 E$ OO
$1.1837164 E$ OI
$5.179204 E$ OO
$-5.3945815 E$ OO
$-5.0001296 E$ O1
$-5.0801296 E$ OI
$5.2215863 E$ OI

$$
0^{2} \mathrm{c}
$$

$1.0235760 E 00$ $6.5262443 E 00$ $6.5262443 E 00$ $.4024245 E$ OI $.0064119 E$ O2 3.9655412E OI $-2.9175713 E$ OI $-4.2214346 E$ O2 $-4.2214348 E$ O2 $4.2969331 E$ O2

CIN
$1.2620115 E-01$
$4.1257001 E-01$
$4.1257001 E-01$
$2.1288195 E-01$
$2.0006161 E$ OO
$1.4055424 E$ OO
$-2.028685 E$ OO
$-1.2440174 E$ O1
$-1.2440174 E$ OI
$1.2970945 E$ OI
ocm 4. $145300 E-0$ l.926750uE 00 2.242575750 $1.9017315 E$ o1 - .6704atse 00 $-9.0313966 E$ OO $-8.5049534 E$ O $-0.5049536 E$ O $0.7417749 E$ ol

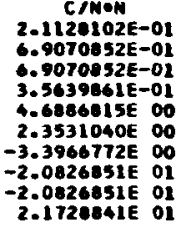

$0.012,0, \cos 1.2 L_{1}-2 L_{2}+0, w_{2}+0 w_{2}^{\prime}$

$$
c \quad 0 C
$$

2. 06: 0

2. 06 0 :

4. 06 0

26, 260 ?

$\alpha_{0}$ ob 0

$\begin{array}{llll}0 . & \text { of } & 1\end{array}$

2.5776796E-01 $-4.67581111 E-01$
$-4.6758111 E-01$ 3.1169384E-01 2.2093487E OO $.5585296 E-01$ -1.2228911600
$-3.2264216 E$

$6.0300775 E-01$ $-5.4167466 \mathrm{E}-01$ $-3.4167466 E-01$ $1.0901857 E$ 00 $9.9820144 E$ OO $3.8561121 E$ OO $-5.0693944 E$
$-3.3427961 E$
$0^{2} \mathrm{C}$

$1.6509552 \mathrm{E} 00$ $1.8977718 E$ OO $1.8977718 E$ O0 $9.0196343 E 00$ ..6566829E 01 3.0031773E ol $-2.7402550 E$
$-3.2904050 E$ C/M
$2.1577250 E-01$
$-3.9140290 E-01$ $-3.91402996-01$ 2.6091200E-01 $1.0494025 E 00$ $5.4900163 E-01$ $-1.0236582 E$
$-2.7007743 E$ 00

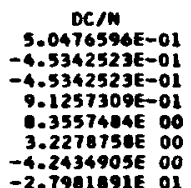

C/MEN $-3.2763575 E-01$ 2.104045e-01 $2.1840490 E-01$ ..5A80985E 0O 4.5955850E-01 $-2.5607451 E$ OO
1. $6061893 \mathrm{E}-01$ 


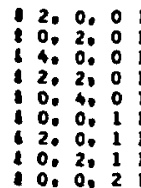

$-1.0530229 E-02$ -. $1532229 E-0$ $.0054129 E$ OD 0.19190 $-3.3270426 E-02$ $1.5862440 E$ OO 1.5842440500 ..5619920E-01

$-9.7798861 E-0$ $9.7798861 E-0$ 5.5361085E oI . $3037027 E$ OI $3.6107422 E-01$ $1.6206092 E$ O (6206992E $.9039151 E$ OO
$-9.1442206 E$ DO $-9.1442206 E$ OO $1.3218260 E$ O2 . $-4.0735682 E$ OO $1.6642972 E$ O2 $1.6842972 E$ O2 $1.6842972 E$.

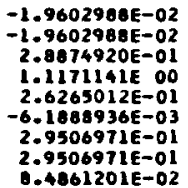
$-1.0192335 \mathrm{E}-01$ 2.6578332E OO 1.0298151E O1 2.425119. 3.0147715E 00 3.014TIISE OO

$3.0147715 E$ OO
$1.0982337 E$ OD

$-3.6465059 E-03$ $-3.6465059 E-03$ 2.3712509E-02 2.07803 $-1.1512446-02$ $5.4888237 E-02$ $9.488837 E-02$ 1.5785699E-02

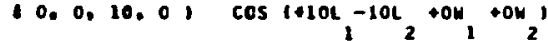

1 0. 0.0

$2.0,0$

1.20 .0

2. 2.0

0.40 .0

$2,0.1$

$10,2,1$;$$
\text { c }
$$

9.7066606E-04

$-6.0036935 E-02$

$-0.6036935 E-02$

$4.8130517 E$ OO

$1.1393861 E$ OQ

$+1.9052081 \mathrm{E}-02$

1. $1500737 E$ oO

1.1586737E OO
2

$1.0092811 E-02$ $-7.0055923 \mathrm{E}-01$ $-7.0055923 E-0$ $1.2703236 \mathrm{E}$ ol 4.9265590 E OI 1.16738996 of 1.3105405E $1.3105405 E$ O
$10.0,11.0, \cos 1+11 L_{1}-11 L_{2}+\mathrm{OH}_{1}+\mathrm{OH}_{2}{ }^{\prime}$

1 0.0.0 0 12.0.0

4. 0.0

2. 2.0

0. 4. 0

2.0.2 1

(0.20, 1 ,

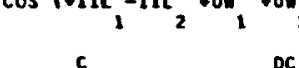

5.0574918E-OC $-4.3181049 E-02$ $-4.3181049 \mathrm{E}-02$ $9.5696471 E-01$ 3.7137173500 0.0404050 -01 $-1.0062757 E-02$ $0.1985176 E-01$ Q.1985176E-01 $5.7654629 E-03$ $-4.8836731 E-01$ 1.0760210 OI 4.1782956 OI $9.9524065 E 00$ $-1.3939380 E-O 1$ .0145510 OL 1.0145510 O
6. 1. $0.012 .0, \cos \left(-11 L_{1}+12 L_{2}-1 H_{1}+0 w_{2}\right)$

c 0 C

1.0.0) $\quad-4.8146128 E-03 \quad-5.9857904 E-02$

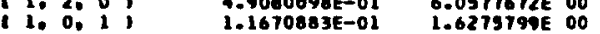

$11,0,-12,01 \cos 1-10 \alpha_{1}+11 L_{2}-1 w_{1}+0 w_{2} 1$

C $D C$

$\begin{array}{rrr}1.0 .0 & -8.4459724 E-03 & -9.6566565 E-02 \\ 3.0 .0 ; & 3.1951210 E-01 & 3.6190734 E 00\end{array}$ 1.8910723E-01 2.4527166E OO
${ }^{2}$

$6.6292945 E-02$

$-5.5563914 E$ OO

$1.2152076 E$ D2

$4.7230970 E$ O2

$1.1260790 E$ O2

$-1.8387735 E$ OO

$1.2773127 E$ O2

$4.0223404 E$ OI
$11.0,-10,0, \cos \left(-x_{1}+10 \mathrm{~L}_{2}-1 w_{1}+0 w_{2}\right)$

120.01

3. 0,0

c

$c$

$-1.47530646-02$

$4.5209260 E-02$

1.03044901 O0
$3.0332401 \mathrm{E}-01$

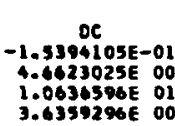

$1.00-9.00$

$\cos \left(-8 x_{1}+9 x_{2}-11,+0 w_{1}\right.$

1.0 .0

$13,0,0$

$$
c
$$

$-2.56366498-02$

$6.2000709 E-01$

1.4374349E 00

1. 0.1 5.767909EE OO $5.2864521 \mathrm{E}$ 00
$O C$
$-2.4190322 E-01$
${ }^{2}$

$-7.3013510 E-0$ 3.3729938E 0 $2.3278105 E$ OI
C/N

$-1.1390477 E-02$

-1.1390477E-O

$2.0796782 E-01$

$.0578223 E-0$

1.9075155E-O

$-3.1890246 \mathrm{E}-03$

$1.9401408 E-0$

$1.9401408 E-0$

$4.7675580 E-02$
DC/N

-

-

$2.1267260 E$ OD

8.247851 EE OO

$1.9543985 E$ OO

-3.777O137E-02

.1940554E OO

$6.6206227 E-01$
$\mathrm{C} / \mathrm{N} \times \mathrm{N}$
$2.720596 \mathrm{TE}-0$.

$-1.90694095-03$ $-1.9069489 E-03$ $3.4817156 E-02$ 1.3490090E-01 $3.1934875 E-02$ $-5.3399444 E-O 4$ 3. $2481076 E-02$ $3.2481076 \mathrm{E}-02$ 7.92165 GE-03
1. $0,-8,0, \cos \left(-\mathrm{r}_{1}+\alpha_{2}-1 \mathrm{H}_{2}+0 \mathrm{H}_{2}\right.$

1. 0.0

3. 0.0

1. 2. 0

$-4.426360$ C -1982769E-01 6.7704832E OO 1.937T913E OO $1.6049230 E$ OI

1. 1. $0 .-1.0, \cos 1-6 L_{1}+7 L_{2}-1 w_{1}+0 w_{2}$,

1.0 .0

1. 2.0

$$
c \text { oC }
$$

-7.5001116E-02 $1.0297605 E$ OO 2.5004213E OO
$-5.6393469 E-01$ 7.445a717E 00 $1.8131406 E$ OI
$1.0372655 E$ OI
C/N $-6.5720016 \mathrm{E}-03$ $-6.5720016 \mathrm{E}-03$ $1.4564661 \mathrm{E}-\mathrm{O}$ .6521452E-0 $1.3454781 E-0$ $-1.6532729 \mathrm{E}-0$ 1.2477851E-0 $1.2477851 E-0$ 2.6757802E-02

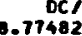

$-7.4327763 E-02$ $-7.4327763 E-02$ $1.4376656 E 00$ 0.3592169600 $1.5147330 E$ OQ $-2.1215240 \mathrm{E}-02$ $1.3441104 E$ OO $1.5441104 E$ OD $3.9710879 E-01$
C/NAN

$-1.0002352 \mathrm{E}-03$ $-1.0002352 \mathrm{E}-03$ 2.2166895E-02 $0.6023633 E-02$ $2.0477697 E-02$ $-2.5162223 E-04$ 1.8990945E-02 $1.0990845 E-02$ $4.0724420 E-03$
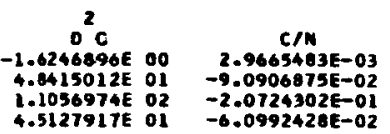

DCr

3.0954485E-02 $-9.3749634 E-01$ $-2.1388081 E$
$-7.3111317 E-01$
C/man

$-5.9651390 E-04$
$1.8279565 E-02$ $1.8279565 E-02$
$4.1672451 E-02$ $1.2269365 E-02$

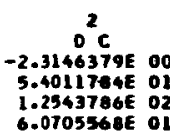
$C / W$
$5.6587014 E-03$
$-1-4162621 E-01$
$-3.2049439 E-01$ $-1.0960689 E-01$

$\mathrm{OC} / \mathrm{M}$ 


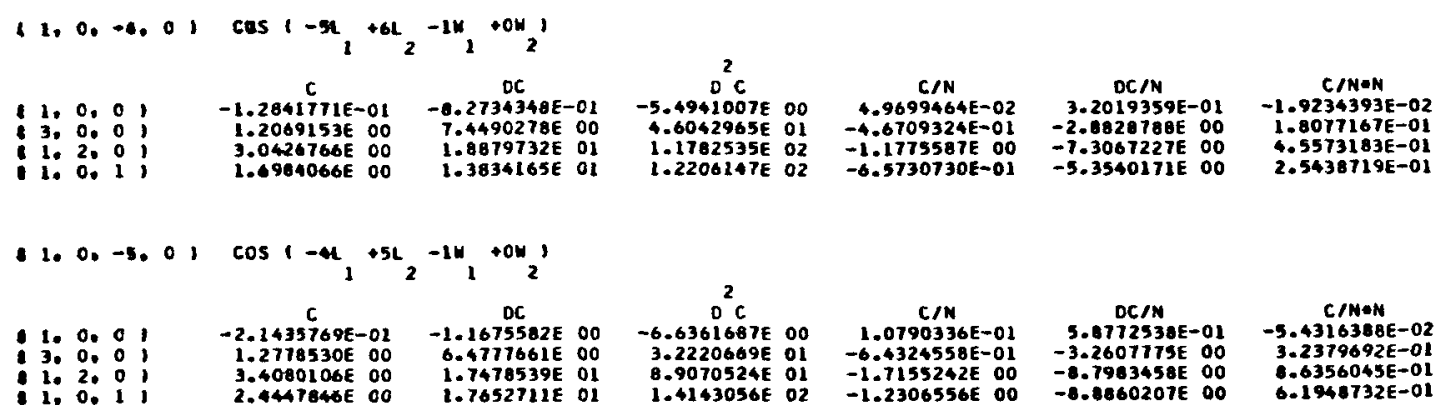

1. 2. O 3.4080106E 0O 1.7478539E OI $8.9070524 E$ OI -1.7155242E $-1.2306556 E$ OO $-8.8660207 E 00$

$6.6356045 E-01$
$6.1948732 E-01$

1. $0 .-4.01 \cos 1-3 t_{1}+4 L_{2}-11,+0 w_{2}$

1. 0.0

1. 2. 0.001

$-3.5014246 E-01$ 1.1402345600 $\begin{array}{ll}3.3615745 E & 00 \\ 3.3605403 E & 00\end{array}$

OC

1. 0. 1 . $-1.5592439 E$
$4.4175655 E$ 1.3394212E OI $1.3394212 E$
$2.1266059 E$ oI

1. $1.0,-3,0, \cos 1-2 x_{1}+3 c_{2}-11,+0 w_{1}$,

1.0.0

3.0.0

i. 0.1 ;

- $\begin{gathered}\text { C } \\ -5.5243430 E-01\end{gathered}$

$7.3730030 \mathrm{E}-01$

$2.6495367 E$ OO

DC
$-1.9136797 E$ OO

$1.6256416 E$ OO $6.9054095 E 00$ 2.3727714E OI

$11,0,+2,0, \cos \left(-16,22,-11,0 w^{\prime}\right.$

1. 0.0

3. $0,0,1$

1. 2, 0.1

$\begin{gathered}C \\ -0.1703980 E-01\end{gathered}-2.0314 \mathrm{DC}$

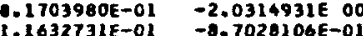

$1.2059996 E$ OO $1.3446341 E-01$

$4.9804784 E$ OO $2.3840063 E$ OL

$11.0,-1,01$ ces $1+a_{1}+11,-14,04$

C

(1.0, $0,1-2.0722506 E-01 \quad-7.7547467 E-01$

i. 2.0? 0 -3.206092E-01 $-1.7595086 E 00$

1. 0.1 3.9091717E 00 $1.9982438 E$ OL

1 1. $0 .-0.01 \cos 1$ the $4 \mathrm{OL}_{2}-1 \mathrm{u}_{1}+\mathrm{Ow_{2 }}$ ?

C $0 C$

$\begin{array}{lll}1.0 .0 & -2.2065981 E-01 & -6.4855371 E-01 \\ \text { 3. } 0.0 ; & -1.4732726 E-01 & -1.0535333 \mathrm{E}\end{array}$

1.2.0, -7.8462422E-01 -3.8380575E OO

Ii. 0.1 3.1377049E DO $1.5352230 \mathrm{E}$ OI

at 1. $0,1,0, \cos 1+2 \alpha_{1}-11,-1 w_{1}+0 w_{2}^{\prime}$

160,0

$3,0,0$

$c$
$-\$ .6461594 E-02$
$5.7900668 E-03$

$3.2900626 E-01$

$1.4854098 E$ DO

DC

OC

$-2.90708806-01$

-2.11224286 OO

$1,0,1$;

$110,2.01 \cos 1 \cdot 3 L_{1}-2 L_{2}-1 N_{1}+0 w_{2} 1$

$1.0 .0,1$ $\begin{array}{cc}C & \text { DC } \\ 2.1403205 E-01 & 3.8053789 E-01 \\ -4.9546309 E-01 & -1.0169179 E-00\end{array}$

3. 0, 0 $-0.6432490 E-01$

$3.8053789 E-01$
$-1.0169179 E-00$

i. 0.1 i $0.8914943 E-02$ 3.56248636 OO

$11.003 .01 \cos 1+4 L_{1}-3 L_{2}-11$, ton 1

1.0 .01

1. 0. 0

i. 2: 1 i
$C$
$1.6593954 E-01$

$-9.5853401 E-01$
$-3.7623154 E-01$
$-7.0922301 E-01$
$-9.5195401 E-01$
DC
DC
$0143 E-O I$ $-2.1153136 E 00$ 7.9286510 E -02
$0^{2} \mathrm{c}$

$D C$
$-7.4035758 E$ $1.5072469 E$ oI 5.0176012E 01

C/N
2.5203581E-01 2.5203581E-OI
$-8.2507027 E-01$

$-2.4196926 E$
$-2.4189482 E$

DCN

1.1223583E OO

-1.53080845 oI
-9.6412705E 00

$-1.0141772 E-01$ $5.9309327 E-01$ l.T4lT173E o0 i.TALIELE OO
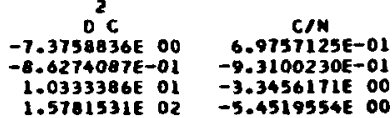

$0 \mathrm{con}$ $-2.0527265 E$ O -9.82060065 OO $-2.9961406 E$ OI

CrNaN

$-0.003562 E-01$ $1.1755931 E$ OO ..8E4281OE DO
$O C / N$

$1.0437004 E$ OI $4.4715009 E$ OO $-6.4007250 E-01$
$-1.2249016 E$
C/NON

-2.13690306 O1 3.0709233E OD 3.1637169E OL 1.314797OE O2
$0^{2} \mathrm{C}$ $-3.3409221 E$ OO $-1.1935819 E$ OI $-2.9239211 E$ O1
$1.2697961 E$ 02

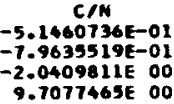

oc/n

$-1.9237562 E 00$ $-4.3694329 E$ OD $-1.0961408 E$ $4.9622902 E$ O1
-1.2779377E OO $-1.9776093 E 00$ $-5.0404209 E 00$ $2.4107496 E$ o1
$0^{2} c$ $-2.4691512 E 00$ $-0.2933327 E$ OO -2.48850406 OI
9.95402136 OI

$0^{2} \mathrm{C}$

-1.29377016 DO -3.92745736 OO $-1.6186719 E$ ol $6.0628185 E$ OI
C/N $-1.4732726 E-01$ $-7.8442622 E-01$
$3.1377049 E 00$
Dc/n

$-6.4055371 E-01$ $-1.0535333 E$ OO -3.8300575E DO 1.5352230E o1
C/MAH

$-2.2065981 E-01$ $-7.8442622 \mathrm{E}-0$ 3.1377049600

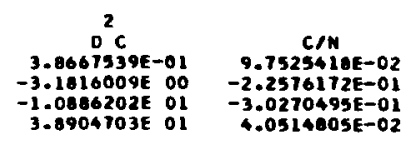

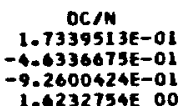

CINAN
4. 4434239E-O2
$-1.0287014 E-01$
$-1.3792994 E-01$
$1.6460895 E-02$
$0^{2} \mathrm{c}$

$1.3143640 E 00$ - 6.660267 OE OO 1.4973299 OI $^{2}$
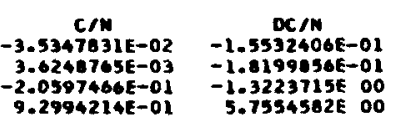

$-2.2129542 E-02$ 2.2693572E-0 5.0219112E-01 
$11,0,4,0, \cos 1+5 L_{1}-4 L_{2}-1 w_{1}+0 w_{2}^{\prime}$

(1.0.0, $\quad \begin{array}{cc}C & \text { DC } \\ \text { 1.0274151E-01 } & 4.1996369 E-01\end{array}$

3.0.0, $-7.7566523 E-01 \quad-3.1223855 E$ 00

1.2.0! $1.1112068 E$ OO $-4.6087291 E$ OO

$-1.1233409 E$ OO

11. $0.0 .01 \cos \left(+61,-5 L,-11,+0 w_{2}\right)$

1. $0.01 \quad 0.4420205 E-02 \quad 3.3181665 E-01$

3.000 : $1.2848634 E-01 \quad-3.6964968 E 00$

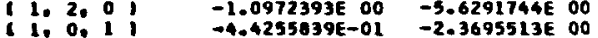

$11.0,6.01 \cos 1+7,-6 L_{2}-1 x_{1}+0 w_{2}$,

$\begin{array}{cccc}C & C & \text { OC } \\ 1.0 & 3.9226276 E-02 & 2.4295374 E-01\end{array}$

3.0.0, $0.4 .2057949 E-01 \quad-3.7953610 E$ OO

1. 20.0

$-9.7176347 E-01$
$-3.4938397 E-01$

$-2.3541062 E$ OO

1. $0,0,01 \cos 1+0,-72,-1 w+0 w$,

$\begin{array}{cccc}\text { 1. } 0.0 & \text { C } & \text { DC } \\ 3.00 & 2.3429362 E-02 & 1.6926958 E-01\end{array}$

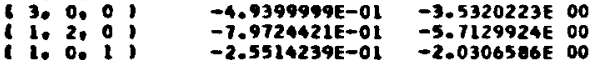

1.0.

1.0.0)

3. 0.0 ?

1. 20,

$\cos 1+9 x_{2}-a L_{2}-1 w_{1}+0 w_{2}$

oc

1.3004679E-02 1.1386568E-01 $-3.1411226 E-01 \quad-3.0593499 E 00$

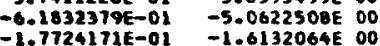
$-1.7724172 \mathrm{E}-01$

1.0 .9 .0$.

$\cos 1010 \mathrm{~L}^{2}-9 \mathrm{~L}^{2}-1 \mathrm{w}, \mathrm{OW}$

(1.0.0)

$3.0,00$

1.0 .1 ,

\subsection{1 $\stackrel{C}{0.05 E-03}$ \\ $-2.7269697 \mathrm{C}^{-01}$}

$-4.5944627 E-01$

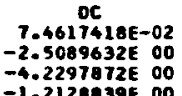

5065

$6.9650659 E-01$ - 2.32015016 01

-1.26180596 ol

$0^{2} \mathrm{c}$

$1.2339126 E 00$

$-2.5395211 E$ OI

$-1.6366325 E$ ol
2

$9.4688033 E-01$ $-2.5154090 E$ O1 $-1.4960115 E$ OI
C/N -3.2786151E-01

$-1.4362006 E-01$
-2.2065077-02
OC/N

-9.2391026E-01 $-1.3598052 E-001$ -9.2125964E-OL
C/NON

. 9441074E-0I -9.6735516E-02 $-6.7525120 E-02$

$11.0,10,0, \cos 1011 \mathrm{~L}-10 \mathrm{~L}-1 \mathrm{w}_{2}+0 \mathrm{w}_{2}$,

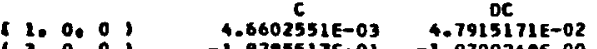

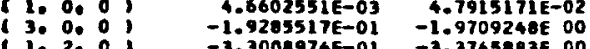

$\begin{array}{llll}1.2,0 & -3.3001974 E-01 & -3.3743883 E & 00 \\ 1.0,1 & -7.7717611 E-02 & -0.7619830 E-01\end{array}$

1 0. 1.-10. $0, \cos (-10$, t11, tow -11,

$\begin{array}{cccc}C & \text { OC } \\ 0.1 .0 ! & 1.5238399 E-02 & 1.5898745 E-01\end{array}$

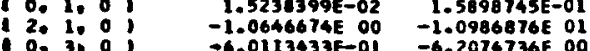

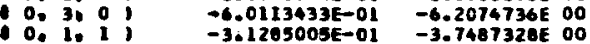

$0^{2} \mathrm{C}$

$1.6777025 E$ 00 $-1.1420074 E$ O2 $-6.4581925 E$ OI
$\mathrm{D}^{2} \mathrm{C}$

$4.9582796 E-01$ $2.0236900 E$ OI $-3.4670419 E$ OI
C/N
$4.5219997 E-03$
$-9.5344715 E-02$

$-1.5387252 E-01$
DC $/ N$

.2669960E-02

$-6.0169973 E-01$

$-1.1026390 E$
$-3.9192829 E-01$
C/Nan

-1.7277083E-04

$-2.9698243 E-02$

$-9.5043410 E-03$
$0,10-9,0, \cos \left(-x_{1}+10 L_{2}+0 w_{1}-1 w_{2}\right)$

$\begin{array}{ccc}\text { C } & \text { DC } \\ \text { 1.: 1.: } & 2.6572470 E-02 & 2.5069546 E-01 \\ \text { 2. } 1.0: & -1.4901260 E \text { OO } & -1.3863002 E \text { OI }\end{array}$

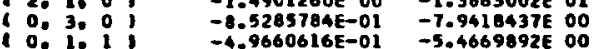

$0^{2} \mathrm{C}$

2.3982800E OO $-1.3000997 E$ O2 $-7.4583541 E$ OI
2.3890018E-03 $-6.4741953 E-02$ $-1.0700395 E-01$ $-3.0672544 E-02$ ocom

1.9705011E-02 $-5.2943543 E-01$ $-2.7917325 E-01$
$4.1342843 E-04$ $-1.8517556 \mathrm{E}-02$ $-5.3080336 E-03$
10. $1 .-0.0, \cos 1-x_{1}+x_{2}+0 x_{1}-1 w_{2}$

C $\mathrm{DC} \quad \mathrm{D}^{2} \mathrm{C}$

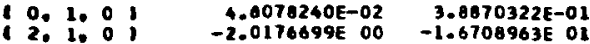

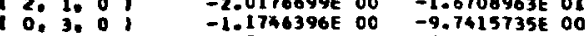
(0.1.1: 1.1 -7.7634248E-01 $-7.7951640 E$ DO $3.3366466 E$ OO
$-1.3957454 E$ O2 $-8.1554048 E$ OI $-8.2337999 E$ OI
C/N
$1.2629244 E-03$
$-4.2770442 \mathrm{E}-02$ $-7.2060646 E-02$ -1.8640046E-02
OC/N

$1.1703173 E-02$
$-3.9351175 E-01$ -

$-1.9023159 E-01$
$C / N=N$
$1.9808007 E$

$1.9808007 E-04$
$-6.7082179 E-03$ $-1.1302163 \mathrm{E}-02$ $-2.9248039 \mathrm{E}-03$
$-1.0530177 E-02$ $4.6109444 E-01$ $2.673625 E-01$
DC/N

6. $8713890 E-03$ $-2.8264515 E-01$ $-4.0394085 E-01$

$-4.7337303 E-02$

$-1.2565330 \mathrm{E}-01$
$C / N=N$
$9.5041296 E-05$ $-3.9661969 E-03$ $-6.7865184 E-03$ $-1.5983151 \mathrm{E}-03$
$-5.3431956 E-03$ $2.9963472 E-01$ $1.7149276 E-01$
$9.9857627 E-02$ 2.7875741E 00
5.0409A74E-02 . $.5969469 E$
1.09930200
CINAN

1.0744104E-03 $-3.4483784 E-02$ $-2.0079304 E-02$ $-6.0250586 E-02$ 
10. $1,-1,0, \cos \left(-7 L_{1}+8 L_{2}+0 n_{1}-1 w_{2}^{\prime}\right.$

:0.1.0?

2., 1.:0 ?

0. 3. 1,1

$\begin{array}{cc}C & O C \\ 7.9345062 E-02 & 5.9012056 E-01 \\ -2.6181950 E \text { OO } & -1.8983075 E \text { OI }\end{array}$ - $1.5592435 E$ OO - $1.8983075 E$ OI -1.190944IE OO -1.0815423E OI

10.16-6.0.1

$\cos 1-6 L_{1}+7 L_{2}+O H_{1}-1 w_{2}$

10. 1.0 ,

2. 1.0 :

$10.3,0,1,1$

35

C

0.7193919E-01

-1.9915189E 01

$-1.9600896 E$ EO
$-1.7837312 E$ OO

$0,1,01$

2. 1.0

:0.1:1 :

$\cos 1-5 t_{1}+6 t_{2}+0 w_{1}-t w_{2}$

2.2829659E-01

$2.2029659 E-01$
$-3.6332731 E$ OO

-2.582560 O

242526

$1.2425269 E$ OO

$-1.8633924 E$ O1

$-1.2020292 E$ OI

$-1.8653824 E$ OI

1 0, 1, $-4,0$

$\cos 1-4 L_{1}+5 L_{2}+0 n_{1}-1 \mathrm{H}_{2}$

10.1.0.

12. 1.0

(0., 1, 1 ,

C
$3.784471 E-01$
$-3.6411233 E$ OO

$-2.4886460 E$ OO

$-3.6009969 \mathrm{E} 00$

OC

$1.6829444 E 00$ $-1.4519396 E 01$ -2.27037476 O1

0.1,-3., 0

$\cos \left(-3 L_{1}+4 L_{2}+0 w_{1}-1 w_{2}\right.$

:0, 1.00,

1. 3. 10

C $773 E-01$

$0.1146773 E-01$
$-2.9502093 E$ OO

-2.27447095 OO

$-4.7087914 E$ OO

OC

QC $2281 E$ OO

$-7.8309109600$

$-6.5087490 E$ OO

$\cos 1-2 \mathrm{~L}_{1}+3 \mathrm{~L}^{2}+\mathrm{OH}-1 \mathrm{H}^{\prime}$

10. 1.0?

2.1.0 1.

0,300

$c$

C $37 a e-01$

$-1.4397901 E 00$

$-1.5234010 \mathrm{E}$ OO

OC

$970 E 00$

$-4.0530074 E-01$
$-1.9118523 E$

$\cos 1-1 L+2 L+0 H-14$

$140.1,+1.0$,

1 0.1.0 1

2.1.0 1

0.3 .03

$$
c
$$

C $9.4509107 E-0$

$4.9214996 E-01$
$-\$ .5151121 E$ OO

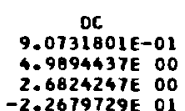

10.1. -0.0,

$\cos \mathrm{C}+\mathrm{OL}+\mathrm{IL}+\mathrm{OW}-\mathrm{IW}_{2}$

1 0. 1.0.

2, 1, 0 1

0. 1. 1 ,

$$
\text { C }
$$

$$
\text { C }
$$

$1.3108254 E$
$1.0017296 E$

$3.5431501 E-01$

-4.0089184 E OO

$0 C$

DC

$.6921352 E-01$ $.6224838 E$
$2.9870164 E$ $-1.8489935 E$ OI

10.1 .2 .01

$\cos \left(+1 L_{1}+0 \mathrm{~L}_{2}+\mathrm{OW}_{1}-1 \mathrm{w}_{2}\right)$

$\begin{array}{lll}0,1,0 & 0 \\ 12, & 1,0 & 0 \\ 0 & 0, & 0\end{array}$

: $0,3: 0$ :

$$
9.4152464
$$

C

$9.4152464 E-02$
$4.5222322 E-01$
$3.9171212 E-01$

$3.9171212 \mathrm{E}-01$
$-2.0913503 \mathrm{E}$ OO

DC

3.7994466E-OI $2.68768306-00$ $2.0801895 E \mathrm{CO}$

10.1 .2 .0

$\left.\cos 1+2 L_{1}-1 L_{2} \cdot \mathrm{Or}_{1}-1 \mathrm{w}_{2}\right)^{\prime}$

$10,1.0,1$

$12,1,0$

: $0,3,0,1,1$,

$C$
$-8.5148072 E-02$
$\$ .3053434 E-01$
$1.8602703 E-01$

$1.8602703 E-01$
$-1.0030049 E-01$

$-7.9034019$

DC

$1.7613979 E 00$

$1.1634731 E$ OO
$-6.0971835 E$ OO
${ }^{2} \mathrm{C}$

DC
$4.4887316 E$ OO
$-1.3881346 E$
-1.32

a.3125340E OI

$-1.0458972 \mathrm{E}$ O2 $C / N$
$-2.0999014 E-02$
$6.9291666 E-01$ $4.1239589 \mathrm{E}-01$
$3.1518852 \mathrm{E}-01$

DC/N

$2.8623486 E$ OD
C/N $=N$

$-1.8338339 E-01$

$-1.0914236 E-01$

$-1.0914236 E-01$
$0^{2} \mathrm{C}$

$5.7862957 E 00$ -1.2427960 OE $-7.7077001 E$ OI

$-7.2780150 E$ O2
1.2503468E-02

$1.0090360 \mathrm{E} 00$

$5.1866276 E-01$

$134 E-01$ $6.2602769 E 00$

$4.5607568 E$ OO

$-3.1718730 \mathrm{E}-01$

$-1.944746 \mathrm{E}-\mathrm{O}$

$-1.7625751 \mathrm{E}-01$
$0^{2} \mathrm{C}$

$7.0540395 E 00$ ..4995205E Ol $-1.2306910 E$ OI
$-8.8353996$

1. $035396 E-02$

8. $0951094 \mathrm{O}^{-}$

$1.0020382 \mathrm{E}$ OO
$\mathrm{DC} / \mathrm{N}$

T.087544E-01

$4.6520286 E$ OO

$7.2192929 E$ OO
C/NAN
3. $4194243 E-02$ $-5.4419131 E-01$ $-3.4812686 t-01$ $-3.8780292 E-O 1$

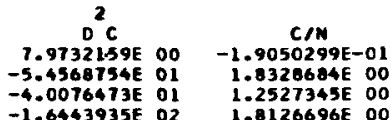

$-1.6443935 E$ O2

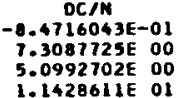

1.1428611E O1
C/NoN

9.5895382E-02 $-6.3060141 \mathrm{E}-01$
$0^{2} \mathrm{C}$

0.1000727e 00 1.2245787 OI $-1.4901535 E$ OI
$-1.6971081 E$ OD

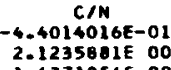

$2.1235801 E$ OO 3.309432OE OO
DC 11

$-1.5201856 E 00$ $5.6367626 E$ OO (.6650382E DO
$\mathrm{C} / \mathrm{N}=\mathrm{N}$

3.1681699e-01 -1.5285785E 0O $-1.1794613 E$ OO $-2.4397447 E$ OO

\section{$D^{2} \mathrm{C}$}

$7.0426231 E$ DO $1.9426175 E$ o1 $6.1506335 E 00$
C/M

1.8180480E OO 1.9238780E OO

7.0610230 EO
DCAN
$-2.9459167 E 00$
$5.1178045 E-01$
$2.4111299 E 00$
$3.3303079 E$ O1
$\mathrm{C} / \mathrm{N}+\mathrm{N}$

$1.5082340 E 00$ $-2.4293149600$ $-8.9160793 E$ OO

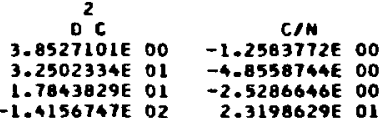

$-1.4156747 \mathrm{~F}$ D2

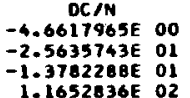

$\mathrm{C} / \mathrm{N}=\mathrm{N}$

$6.4655374 E$ DO $2.4949464 E$ OI $1.2992269 E$ OI -1.1919447 E 02

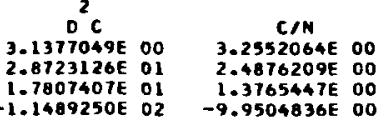

OCA $1.1479133 E$ OI $-4.5916531 E_{01}$
C/NON

- 0837377200 $3.4184089 E$ OO
-2.4710292 OI 


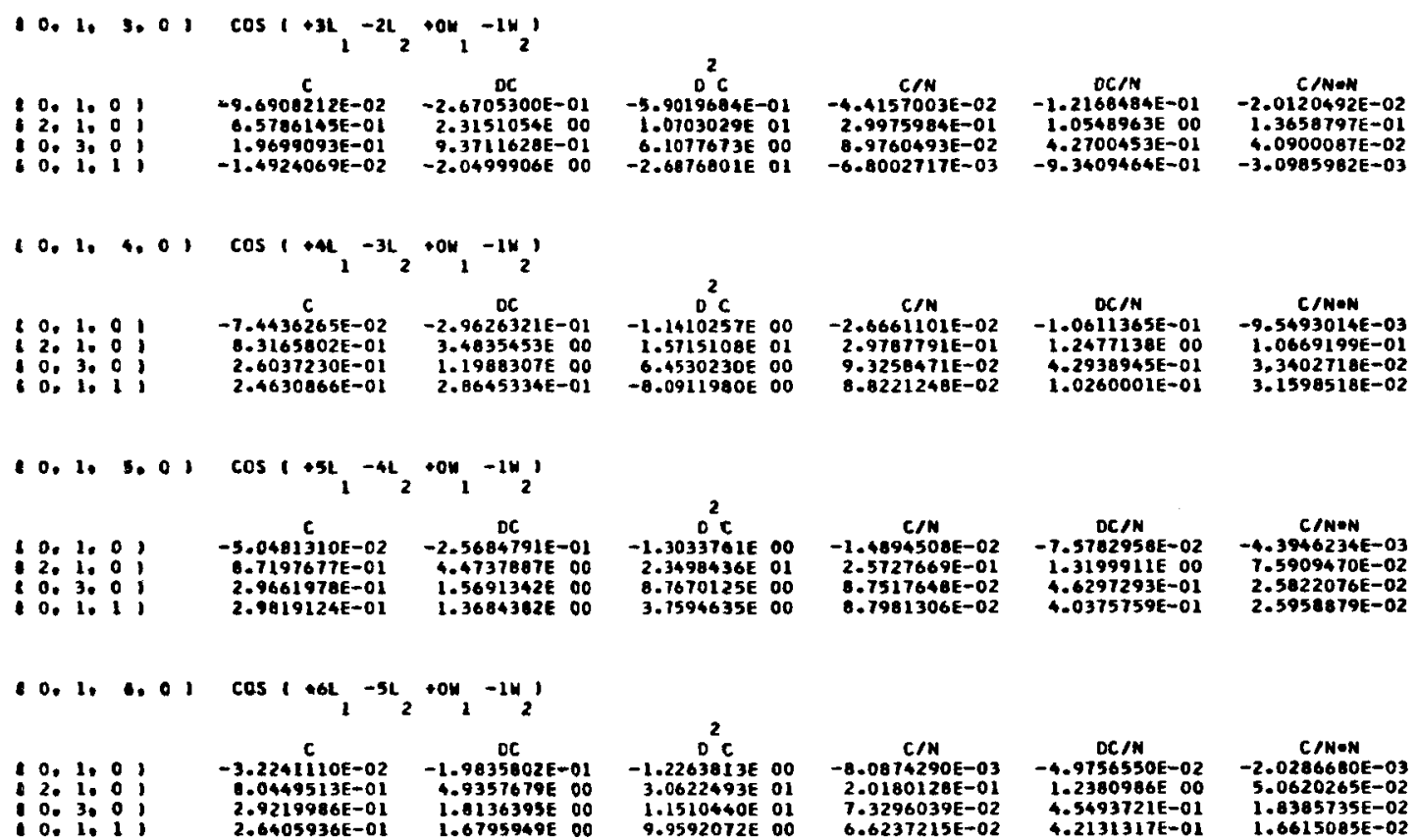

10. $1.7,0, \cos 1+7 L_{1}-6 L_{2}+0 w_{1}-1 w_{2}$

10.1.01 -1.9805436E-02 -1.4308371E-01

2. 1. 0 - $1.7947044 E-01$ T.861403EE 00

0.3. 0 ; $\quad 2.5907455 E-01 \quad 1.0629579 E 00$

$9.9592072 E$ DO

$6.6237215 \mathrm{E}-02$

$4.2131317 E=01$

$1.6615005 E-02$

10.1. 1, 0, $\cos 1+0 L_{1}-7 L_{2}+0 w_{1}-1 w_{2}$

$\begin{array}{rrr}C .1 .0, & -1.1990239 E-02 & -9.8636315 E-02 \\ 1.1 .0, & 5.3844519 E-01 & 4.4025170 E \text { DO }\end{array}$

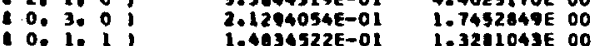

$0^{2} \mathrm{C}$
$-1.0365800 \mathrm{OE}$ OO
$3.5045458 \mathrm{OA}$
$1.3556880 \mathrm{OI}$
$1.2195427 \mathrm{OI}$

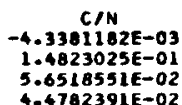

oC $/ N$

$-3.1214306 E-02$ $4.0641459 E-0$ $3.4636345 E-01$

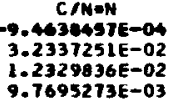

$10.1 .0 .0, \cos 1 * x_{1}-8 L_{2}+0 w_{1}=1 w_{2}$

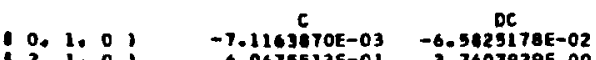

$4.0675513 E-01 \quad 3.7407929 E 00$

$\begin{array}{lll}0.3 .0,1.6545413 E-01 & 1.5235560 E & 00 \\ 0.1,1, & 1.0226152 E-01 & 1.0323468 E\end{array}$

$D^{2} \mathrm{C}$

$-6.1286368 E-01$ $3.4586000 E$ OI $1.4116620 E$
$1.0581875 E$

C/M

$-1.2315255 E-03$ $7.0390961 E-02$ $2.8633337 \mathrm{E}-02$ $1,7696055 E-02$

$\mathrm{OC} / \mathrm{M}$

$-1.1391369 \mathrm{E}-02$ $6.4736248 E-O 1$ $2.6365881 \mathrm{E}-01$ 1.7865265E-01

C/NON

$-2.1312150 E-04$ $1.2181500 E-02$ $4.9551380 E-03$ 3.0625272E-03

10.1. 10.0 I $\cos 1+10 \alpha_{1}-9 L_{2}+0 w_{1}-1 w_{2} 1$

$0.1 .01-4.1749221 E-03 \quad-4.206076$ $766 E-02$ .12749221E-03 $-4.2868766 E-02$

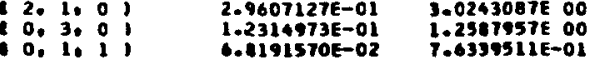
$D^{2} C$
$-4.4281502 E-01$
$3.1039415 E$ O1
$1.2934146 E$ O1
$0.6868434 E$ 00 C/N DC/N .6.5480472E-04 $4.6436522 E-02$ $1.9315096 \mathrm{E}-02$ $-6.1236392 E-03$ $4.7433977 E-01$ $1.97432525-01$ $1.1973270 E-01$

C/NON

$2.4664990 E-04$ $7.9322745 \mathrm{E}-03$ $5.5260264 E-03$

- 2. $0,-10,0, \cos \left(-\mathrm{u}_{1}+10 \mathrm{~L}_{2}-2 w_{2}+0 w_{2}^{\prime}\right.$

2.0 .0

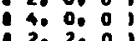

2, 0.21 i

12.0, -0,0)

$\cos 1-\pi_{1}+x_{2}-2 w_{1}+0 w_{2}$

$12,0,0$

$2,2,0 ;$

2. 0, i ;$$
\text { OC }
$$

$1.0239913 E-01$ $-2.2219245 E$ OO $-2.7016688 E$ O1

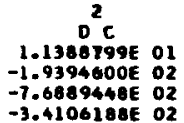

C/M

$2.5772833 \mathrm{EE}-02$ $4.5434873 E-01$ $1.7931534 E$
$5.5923612 E-01$
OC/N

$-2.7007287 E-01$ $4.6929361 E$ OO $1.8555508 E$ O1
C/MEN

$0.4667636 E-03$ -1.1435502E-OI -1.4075412E-0I 
2. $0,-6,0) \cos \left(-6 \alpha_{1}+a L_{2}-2 w_{1}+0 w_{2}\right.$

$20.0,0, \quad 2.4085444 E-01 \quad$ DC

$4,0.01$

$2,2,0,1$;

$-2.4457750 E$ OD

$-1.0469886 E$ OL $-8.6999724 E$ O1

$-8.6999724 E$
$-4.4825298 E$ O1

$2,0,-3,0, \cos \left(-x_{1}+x_{2}-2 w_{1}+0 w_{2}\right)$

2.0.0 0

4. $0,0,1$

$2,2,0,1$

$c$
$3.5563065 E-01$
$-2.5496110 E$

$-2.5401110 E$ OO

$-1.1615134 E$ OI
$+5.0183450 E$ OO

DC

DC

-1.8389804E OI

$-6.4502919 E$ OI

$-5.4404150 E$ OI

$\begin{array}{rl}D^{2} C & \\ 2.05751436 & 01 \\ -1.3301698 E & 02 \\ -6.1992456 E & 02\end{array}$

$-6.1992456 E$ O2
$-5.4464780 E$ O2

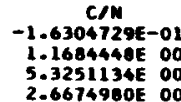

OC/N

$-1.2238650 E 00$

8.4310514E 00

$2.4942309 E$ ol

C/NAN

7.4751207E-02

$-5.3568910 \varepsilon-01$ $-2.4413694 E$ OO $-1.2229501 E$ OO

1 2. $0,-6,0, \cos 1-4 L_{2}, 6 L L_{2}-2 W_{1}+0 w_{2}$,

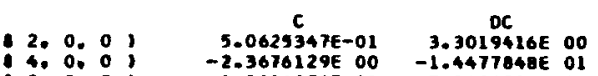

$-1.1816858 E$ OI

$-1.4477848 E$ O1

$-7.4079432 E$ OO

$-7.3488920 E$ O1
$-6.2654009 E$ O!

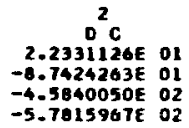

$C / M$
$-3.1962763 E-01$
1.4948134600
$7.4606783 E$ OO
$4.6770707 E$ OO

OC/N

-2.0841102E 00

9.1407162E 0

$4.6397883 E$ O

$3.9557165 E$ OI

$C / N \oplus N$

$2.0179974 E-01$ $-4.7103655 E$ OO $-2.9529102 E 00$

2. $\left.0,-5,0, \cos 1-x_{1}+5 L_{2}-2 w_{1}+0 w_{2}\right)$

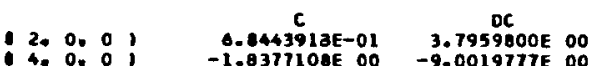

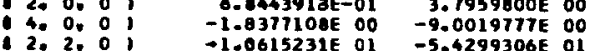

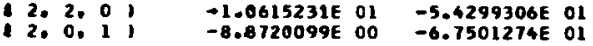

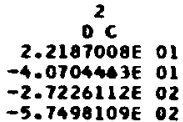

C/N

SSTE-O

$1.8627253 E$ OO

$1.0759723 E$ O1

DCA

$-3.0476501 E 00$

9.124510BE OO

$5.5038416 E$ O

6.0420087 OI
C/MON $-1.8880803 E$ OO $-1.0906102 \mathrm{E}$ OI
1 2, $0,-4.0, \cos \left(-2 L_{1}+4 L_{2}-2 w_{1}+0 w_{2}\right.$

12. 0. 0

4. 0.0

2. $2,0,1$,

- 2, $0,-3,01$

- 2.0.0 01

400,0

2. 2.0

$2,0,+2,0$,

$\cos \left(+0 \mathrm{~L}_{1}+2 \mathrm{~L}_{2}-2 \mathrm{~N}+\mathrm{OW}\right.$,

2.0.0, 0

2. 0.11

C $521 E-01$

2.7655529E-01

$-4.5201102 E-01$

$-7.5108585 E$ OO

2.21291

$2.2129116 E 00$

2.050287E OO

-4.4356090 O O1

at $2,0,-1,0$,

$\cos 1+1 L_{1}+1 L_{2}-2 w_{1}+$ ow $w^{\prime}$

12. 0.0

$40.0,0$

: $2: 0.1$;

15140

$512 \mathrm{E}-01$

$1.4789041 E-01$

$-4.1698017 E$ OD

OC

$0.1432268 E-01$

$1.1739827 \mathrm{E} 00$

$6.3501621 \mathrm{E} 00$

$12.01-0,01 \cos 1 \cdot 2 L_{1}+\mathrm{OL}_{2}-2 \mathrm{H}_{1}+\mathrm{OH}_{2}$

(26,0, 0 )

400,0

$2,2,0$

$-3.35642$

$294 E-03$

$4.4461122 \mathrm{E}-02$

$3.7119471 E-01$

OC
$1.3587252 E-01$
$4.4328648 E-01$

$4.4328648 E+0$
$3.3427239 E$

$-1.3370896 \mathrm{E}$ OI

01200.120

$\cos 1+3 \alpha_{1}-1 L_{2}-2 w_{1}+0 w_{2}$

2. 0.0

4. 0. 0

2. 2. 0

$-1.797937$

C

$4.2951174 E-02$

$.5999635 E-02$

OC

OC

$2.2624661 E-01$

$1.2625162 E 00$
$-4.9911299 E 00$
$0^{2} \mathrm{C}$

$1.4468791 E$ O1

$1.5136147 E$ OL

$1.3173353 E$
-4.43350706

$4.5036646 E 00$ - . $2492597 \mathrm{E}-01$

- .
.6475424E O 3.T312537e $-2.8226654 E$ O
C/MEN

$2.1646260 E$ O1 -3.9640962E OO -9.1457684E O1
2

6. 4042478E 0O 1.7066553E O1 $5.8184032 E$ ol
C/N $3.4338843 E-O I$ $3.4338843 E-0$
$-5.6124529 E-0$ $-9.6124529 E-01$ DC/N
$2.747691 E$ OO
$2.4850401 E$ O0
$5.5257571 E$ O0
$-5.5075309 E$ OI
C/NON

$1.1972667 E 00$ $4.2637267 E-01$ $6.9687744 E-01$
$0^{2} \mathrm{c}$

$3.6469642 E$ OO 1.08690706 OI 5.2767935E O1 -2.2201264 O2
C/N

$1.0799647 E-01$ $1.0543374 \mathrm{E}-\mathrm{Ol}$ 2.9727269500
DC/M
$4.8073600 E-01$
$0.3695341 E-01$
$4.5271450 E$ OO -1.9550790 OI
CINAN

7.6992632E-02 T.5165612 E-02 $-2.1193106 E$ DO

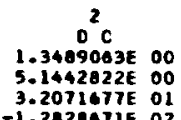

$3.2071677 E$ OI
$-1.2828071 E$ O2

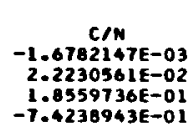

$\mathrm{DC} / \mathrm{N}$

6.7936259E-02 $2.2164324 E-01$ $1.6713620 E$
$-6.6854679 E$
$O O$
$C / N \otimes N$

$-6.3910733 E-04$ $1.1115200 \mathrm{E}-02$ $9.2798679 E-02$ $-3.7119471 E-01$

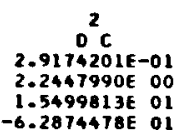

C/N
$-6.9203708 E-03$
$1.6536765 E-02$
$3.3110602 E-02$ $3.3110602 E-02$
$-1.1168129 E-01$
OC/N

$-7.5635737 E-03$

C.7107909E-02

$4.8600520 E-01$

$-1.9216504 E$ OO
$\mathrm{C} / \mathrm{N}=\mathrm{N}$

$-2.6644334 E-03$ $6.3668711 \mathrm{E}-03$ $1.2740016 \mathrm{E}-02$ 
12. $0.2,0, \cos \left(40,-2 L_{2}-2 w_{1}+0 w_{2}\right.$

$\begin{array}{cccc} & & c & \text { OC } \\ 12.0 .0 & 2.1497963 E-01 & 4.1852051 E-01\end{array}$

$\begin{array}{llll}1.0 .0 & -5.1387643 E-01 & -9.1296065 E-01 \\ 2.20 & -5.3761448 E-01 & -6.9944246 E-01\end{array}$

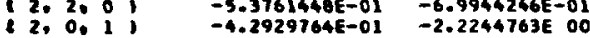

$0^{2} C$
$0.7069506 E-01$
$-8.9606065 E-01$

$\$ .2014797 E$ OO
$-2.7254267 E$ OL

$C / N$
$6.7294096 E-02$
$-1.6095640 E-01$

DC/

1.3100757E-O

$-2.8577992 E-01$
$-2.1894329 E-01$

$-6.9631770 E-01$
C/NON

2.1064765E-02 $-5.0352148 \mathrm{E}-02$ $-5.2678120 E-02$
2. $0.3,01 \cos 1+5 L_{1}-3 L,-2 W,+0 w_{2}$

$12,0,0$

4. 0.0

$2,0.1$,

$c$
$1.9026511 E-01$

$1.9026511 E-01$
$-8.2920502 E-01$

$5.6964540 E-01$ 8.2920502E-01 -2.4193180E OD $-5.7297639 E-01$

$-2.0869991 E$ OO

120. $0.4 .0, \cos 146 L_{1}-4 L_{2}-2 W_{1}+0 H_{2}$
2., 0.0,

$4,0.0$

2. 0.1

$$
\text { C OC }
$$

$\begin{array}{cc}C & D C \\ 1.4924299 E-01 & 6.0353322 E-01\end{array}$ $-1.0161242 E$ OO $-4.0496701 E$ OO

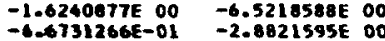

$\left.12,0,5,0, \cos 1 \pi L_{1}-5 L_{2}-2 w_{1}+0 w_{2}\right)$

2. 0.0 :

1.0001

2. 20, 0 : 1 ;

\section{DC}

1.00907age-01 5.5438146E-O $-1.0627527 E$ OO $-5.3489276 E$ OO

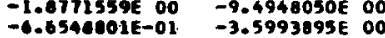

1260. $0.0, \cos \left(40 \alpha_{1}^{-61}-2 w_{1}+0 w_{2}\right.$

20.0, 0

$4,0,03$

2. 20, 0.1 :
$C$
T. $5892351 E-02 \quad 0 C$

T.5e82351E-02 4.6453890E-O $-1.0000541 E$ OO $-6.0759221 E$ OO $-1.9004044 E$ OO
$-5.9367442 E-01$
$20^{2} \mathrm{C}$

$\mathrm{s}^{2} \mathrm{c}$

$1.7157170 E$ OO
$-6.7478848 E$ OO $-6.7478868 E$ OO $-1.6261200 E$ O1

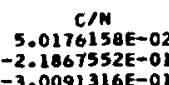

$-2.1867552 E+0$ $-1.5110365 E-01$
DC/

1.5022522E-0 $-6.3801546 E-0$ $-8.7643249 E-01$
$-5.5035092 E-01$
C/NON

$1.3232309 \mathrm{E}-02$ $-5.7668468 E-02$ $-7.9355935 E-02$
$-3.9848613 E-02$

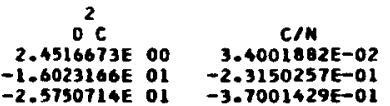
-1.46IILTEE OI
DC/N

$1.3750237 E-01$ $-9.2263229 E-01$ $-1.4858686 E-00$
$-6.5663952 E-01$
CIN $=\mathrm{N}$

$1.7466148 E-03$ $-5.2742999 E-02$ $-8.4299985 E=02$
$-3.4637567 E-02$

120 $0.7,0, \cos 1+x_{1}-r_{2}-2 w_{1}+0 w_{2}$

26. $0.0, \quad 5.1055375 E-02 \quad 3.6509230 E-01$

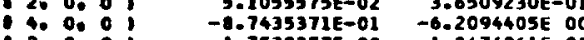

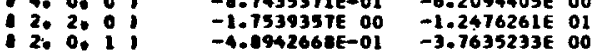

$0^{2} \mathrm{C}$ 2.8357a37E 00 $-2.6934295 E$ ol $-4.8082624 E$
$-2.0353284 E$

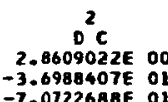
$-3.6988407 E$ OI $-2.6086683 E$ OI
C/N
$2.1856279 E-02$
$-2.1312295 E-01$
$-3.7644223 E-01$ $-3.7644223 E-01$
$-1.3345203 E-01$
DC/N

$1.1117488 E-01$ $-1.0726665 E 00$ $-1.9040750 E$
$-7.2181655 E-01$
C/MON

4. $3830277 E-03$ $-4.2739379 E-02$ $-7.5491199 E-02$ $-2.6762284 E-02$
2. $0.0 .01 \cos 1+10 \alpha_{1}-x_{2}-2 x_{1}+0 n_{2}^{\prime}$

$\begin{array}{cccc}C & \text { C } & \text { OC } \\ \text { 2. } 0.0 & 3.3477479 E-02 & 2.7368115 E-01\end{array}$

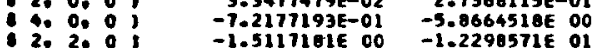

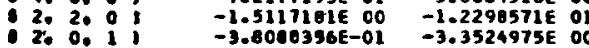
$2600.010 \cos \left(411 L_{1}-9 L_{2}-2 N_{1}+0 w_{2}\right)$

2.0.0.1

40.001

2. 20.0 $1:$ $c$ $2.1514081 E-02$
$-5.6997787 E-01$
$-1.2360911 E 00$
$-2.8355764 E-01$

\section{DC}

1.9783226E-01 $-5.2153696 \mathrm{E}$ O0 $-1.1316618601$

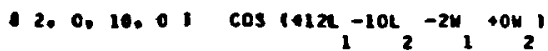

2.0.0.

4.0 .0

2. 0,1

$$
\text { C } \quad \text { DC }
$$

\section{DC}

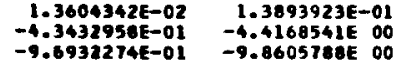
$\begin{array}{ll}-9.6932274 E-01 & -9.8605788 E \\ -2.0401158 E-01 & -2.2394550 E\end{array}$

1. $10-12,0) \cos \left(-11 t_{1}+13 L_{2}-1 \|_{1}-1 w_{2}\right.$

1. 1. 0

3. 1. 1.0

i. 1,0

$$
c
$$$$
\text { DC }
$$

$$
\begin{aligned}
& 9.0111651 E-02 \\
& 4.1134400 E 00 \\
& 5.0707087 E 00 \\
& 2.2726503 E 00
\end{aligned}
$$

$-1.1233023 E 00$ $5.0847714 E$ OI $6.2729620 E$ OI
$0^{2} \mathrm{C}$ 2.6260576E OO $-4.4254621 E$ O -8.913985 IE O -2.9471050 OI
C/N
$1.3589526 E-02$
$-1.7923974 E-01$ $-1.1923974 \mathrm{E}-01$ $-1.0631960 E-01$ ocrm

B.3192774E-02 $-1.0881173 E$ OO $-2.0719838 E$ OO
$-6.9688431 E-01$
CrNew $2.4337044 E-03$ $-3.2099467 E-02$ $-6.0949910 E-02$ $-1.9040434 E-02$
$0^{2} \mathrm{C}$

2.2496811E OO $-4.1039334 E$ OI $-1.0040120 E$ O $-3.0013974 E$ OI
C/N

$8.25981608-0$ $-1.4145373 E-0$ $-2.8375330 \mathrm{E}-0$ $-7.9179089 E-02$
DC $/ \mathrm{N}$

5.9064960E-02 $-1.0045689 E 00$ $-2.0184208 E$ DO $-6.0886610 \mathrm{EF}-01$
$\mathrm{C} / \mathrm{N} \bullet \mathrm{N}$

1.3362006E-03 $-2.2804512 E-02$ $-4.5905864 E-02$ $-1.2809794 E-02$
$0^{2} \mathrm{C}$

1.02836600 OO $-4.71955040 E$ OI $-1.0401738 E$ O2
C/N

4.9387639E-0 $-1.0647938 E-01$ $-2.2301616 E-O$ $-5.6178033 E-02$
DC/N

4.0374103E-02 $-8.6544811 E-0$ -1.0143463E OO $-4.9457707 E-01$
C/NAW

$7.2859100 E-04$
$-1.5708367 E-02$ $-1.5708367 E-02$ $-3.2900452 E-02$
$-8.2876625 E-03$
C/N $-7.7276461 E-02$ $-1.6758676 \mathrm{E}-01$ $-3.8444178 E-02$
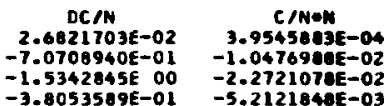
1. $1 .-11.01 \cos 1-10 \alpha_{1}+12 L_{2}-1 H_{1}-1 \omega_{2}$,

$\begin{array}{cccc}C & C & D C \\ 1.1 .0, & -1.4541197 E-01 & -1.6678072 E & O 0\end{array}$

1.1.0 $\quad 5.4839258 E$ OO

1. 1. 1 i $3.4020124 E$ OO

$\mathrm{D}^{2} \mathrm{C}$

$-1.9329053 \mathrm{E} 01$ $0.9661006 \mathrm{E}$ O

$6.0286883 E$ O2

$2.8138240 E$

$-1.0611783 E 00$

$-1.3342145 E$ OO

$-6.5831338 E-01$
${ }^{O C} / \mathrm{N}$

$3.2273244 E-01$ $-1.2044659 \mathrm{E}$ ol $-8.6261309 E$ OO
C/N=N

$-5.4449478 E-03$ $2.5017991 E-01$ $1.2738828 E-01$
$11.10+10.01 \cos \left(-9 x_{1}+11 L_{2}-1 w_{1}-1 w_{2}\right.$,

1. 1.0 1

1.1. 1.0 1

1. 3. 0

$1,1,-9,0$

1 1. 1,001

$13.1,0,1$

1 1. 1: 1

$11.1 .-6.0$

$\cos \left(-n_{1}+9 t_{2}-1 w_{1}-1 w_{2}\right)$

1.1. 1.0 1

3.1 .0 ;

1. 3.0

$C$

C $753 E-0$

B. $5857652 E$ OD

$1.4267903 E$ O1 $1.1870961 E$ OD

$1.0108322 E$ O1 $1.0353031 E$ O2

1.1. $1 .-7,0$

$\cos \left(-6 L, 81,-1 \omega,-1 w_{1}\right)$

1. 1. 01

$13: 1.0$

$1.3,01$

1. 1. 1 ,

C
$-8.5047572 E-01$
$1.1448141 E$ ol

$1.6569906 \mathrm{E}$ O1

-6.3752097E OO

8.2943541E O1

$1.2083350 E$ O2

$1.2600282 \mathrm{E}$ O2

$11.1 .-4.01 \cos \left(-5 x_{1}+7 L_{2}-1 x_{1}-1 w_{2}\right.$

1.100,

$3.1,0$

1. 3, 0,1

C
$-1.2403869 E$
$1.1569463 E$

$1.7939129 E$
$1.7956726 E$

OC

$-0.1247830 E$ OO

$7.1440163 E$ OI

1.1210560 O

$1.5095201 \mathrm{e} 02$

$11010-5,01 \cos 1-4 L_{1}+6 L_{2}-11 x_{1}-1 w_{2}$

1.1.0 1.0

1.1.0.

C
$-1.7627464 E$ OO
$1.0267074 E$ OI

$1.7631426 E$ OI

2.2272671E ol

OC

$-9.7396545 E 00$ $5.1738048 E$ OI $9.1254952 E$ o $1.6780519 E$ O2

11.1 .1

$11,1,-4,0, \cos 1-3 t_{1}+5 L_{2}-1 w_{1}-1 H_{2}$,

1.1. 1.0.1

$$
\text { C }
$$

$-2.3552630 E$ OO

1.3668380E OO

$1.5019615 E$ OI
$2.5755861 E$ OI

$-1.07183$

2.74152796 OI

.0558486E

1.7303466 E 02

1. 3. 1.0
$0^{2} \mathrm{C}$

$-2.5761913 E$ O

$7.6184195 E 02$

$\begin{array}{lll}9.8488173 E & 02 \\ 7.6548027 E & 02\end{array}$
$-1.5486694 E 00$

$-1.9943480 E 00$
$-1.0946100 E$ OO
OC/N $-1.6007523 E$ OI $-2.0646877 E$ OI
C RNEN

$-1.1100433 \mathrm{E}-02$ $3.3884350 E-01$ $4.3635643 E-01$ 2.3949607E-O1
$0^{2}$

$-3.3272047 E$ OL 7.6823930E O2

$9.4260156 E$ O2

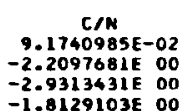

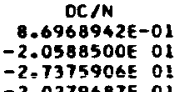

$-2.02796870^{2}$
CINEN

$-2.3090286 E-02$ $5.5617645 E-01$ T.3778966E-OI 4.5629134E-OL

\section{$0^{2} \mathrm{C}$ \\ $4.1360934 E$ OI \\ $7.1735536 E$ O2 \\ $1.9765294 E$ O}

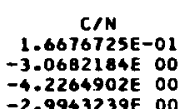

$-2.9943239 E$ OO

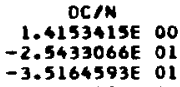

$-3.5164593 E$ OI

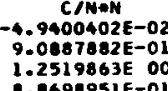

..8698951E-01
$0^{2} \mathrm{C}$

$-4.9042020 E$ OI

$6.0463678 E$ O2

8.902617LE O

$1.2759351 E$ o3
$C / N$

3.060910E-01

-5.9035866E 00

-4.9635666 OO
OC/M

$2.2944677 E$ DO 2.9051767 OI 4. 3488538 ol

4.0068812E O1
$\mathrm{C} / \mathrm{N} \bullet \mathrm{N}$

$1.1016328 E-01$

$1.4026343 E 00$

1.1.003206E OO

$11,1,-3,0, \cos \left(-22_{1}+4 L_{2}-1 \omega_{1}-1 w_{2}\right)$

C DC DC 215 OI

3. 1.: 1.0 3.3933721E vo $5.2583752 E$ 0O

1. 1. 1, 2.6975382E o1 $1.6195466 \mathrm{E}^{02}$

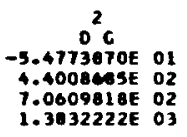

$$
0^{2} \mathrm{C}
$$

-5.6601476E OI 2.5146301E 02 $4.7018836 E$ O2 $1.4137193 E$ O3 $C / M$
$5.1233963 E-01$
$-5.3041730 E$ OO $-8.2244335 E$ OO $-8.2325008 \mathrm{OO}$

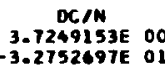
$-3.2752697 E$ OI $-5.1396311 E$
$-6.9206334 E$ 01
CAMEN

$-2.6239675 E-01$ 2.4317699E 00 $3.7706014 E$
3.7742999
1. 1. -2. $01 \cos 1-1 t_{1}+3 L_{2}-1 x_{1}-1 w_{2}$

C $C$ DC

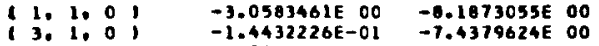

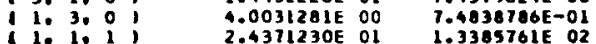

1. 1. 1
$0^{2} \mathrm{c}$

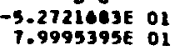

$2.29029060_{02}$

$1.34760700^{03}$
O.

C/N

$2.3873224 E 00$ $-7.4671139 E$ OO -2.6106444E O1
oC/N

$1.0064202 E$ OI $-2.7780450 E$ OI $-6.1382795 E$ oI
CAMAM

-2.419E1E1E 00 $7.5607546 E$ OO 1.5431286 E 01 $2.6461800 E$ O1
$0^{2} \mathrm{C}$

$-4.2603066 E$ O1 $-3.4715064 E$
$3.7530520 E$ $1.1844769 E^{03}$
C/M 7.71756066 OO $-2.5889816 E$ OI $-6.9300219 E$ OI
DC/M

2.6681154E OI -1.35087s6e O $-6.9303899 E$ OI $-4.1606120 E 02$

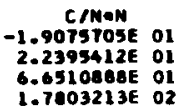


$\left.\because 1,1,-1,0, \cos 1+\mathrm{OL}_{1}+2 \mathrm{~L}_{2}-1 \mathrm{H}_{1}-1 \mathrm{H}_{2}\right)$

1. $1,0,10$ DC

1.1.0, $-6.5857455 E-01 \quad-2.8336730 E 00$

1, 3, 0,0 - $1.3607137 \mathrm{E} 00$-8.6071725E OO

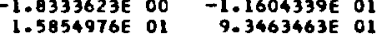

$0^{2} \mathrm{C}$

-1.37198786 OI $-6.7406991 E$ O1
$-8.9786263 E$ ol $-8.9786263 E$
$6.9069057 E$

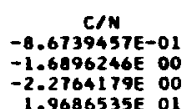

2.27641796 OO
$O C M$

-1.06408677E OI 1.1605011 O2
$C / M+M$

-1.0770116e 00 -2.0979441E 00 2.0265435e 00
1. $1,-0,0, \cos 1+11,+1 L_{2}-1 \omega_{1}-1 \omega_{2}$

1. 1.0 $C$
$-4.3460676 E-01$
$-6.0043029 E-01$

3.1. 1.0

1. 3, 1,1 $-6.0043029 E-0$ $-1.4935082 E 00$
$9.2449676 E 00$ DC
$-1.5688525 E$ OO
$-4.6734330 E$ OO
$-8.9037037 E$ OO $-8.9037037 E$ OO
$5.7466252 E$ OI

1. 1. 1. $0, \cos 1+2 L+0 L,-1 \omega,-1 \omega$,

1. 1.0

3.1.0

1. 3, 0,1

C
$-9.581906 E-02$
$-1.4824947 E-01$
$-0.4831262 E-01$

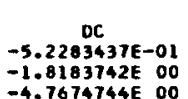

$3.8539327 E$ OO $-4.7674744 E$ OO $2.9717456 E$ OI

1.1.2.01

$\cos 1+3 L_{1}-1 L_{2}-1 \omega_{1}-1 \omega_{2} 1$

1. 1.0

3.1.0

$1,3,0$

$c$
$-1.3077914 E-01$
$2.3973571 E-01$

$2.3473571 E-01$

$-2.09692495-01$
1.64767078 OO

OC
$-3.7746913 E-01$
$-6.5423669 E-02$
$-2.0689720 E$ OO

$-2.0688720 E$ OO
$1.4108622 E$ OI

1. 1. 3. $01 \cos 1+4 L_{1}-2 L_{2}-1 \alpha_{1}-1 w_{2}$

1.1 .0

3. 1, 0 )

1. 1, 1 ;

6
$-1.5719019 E-01$
$1.15400735-01$
$1.22416646-01$
$9.802 \pi 3108-01$

$-5.06060$

$-5.0606059 E-01$
$1.9861601 E$ OO

$-2.4253483 E-01$

$7.2864285 E$ OO

$11.1,4,01$

$\left.\cos 1+5 x_{1}-3 t_{2}-1 w_{1}-1 w_{2}\right)$

1.1, 1,

3. 1, 0

1. 1. 1

C

C $345 E-01$

$1.1536355 E$ OO

$.4207385 E-0$
$8.4200798 E=0$

DC

$-6.1454002 E-01$ $4.5936608 E$ DO 1. $5688113 E$ OO

5.1914123E OO

1.1. 1.5 .01

$\cos 1+6 L_{1}-4 L_{2}-1 w_{1}-1 w_{2}$

1.1.0.

$3,1,0$

1. 3,

$-1.239926$

C $260 E-0$

$1.4299401 E$ OO

$6.9853185 \mathrm{E}-0$

$D C$

$-6.3255147 E-01$

$7.2065606 \mathrm{E} 00$ $3.4621645 E$ OO

$4.9624591 E 00$

$1.1,0.01$

$\cos 1+7 L_{1}-5 L_{2}-1 w_{1}-1 n_{2}$

1. 1.0 $-9.426705$ C
$650 E-02$
$067 E 00$ $1.5155067 E$ OO

$0.4637944 E-01$
$7.4455873 E-01$

oc

3.1.0

1.3.0 1.1

OC $-5.7695748 E-01$ .2046161E OO $5.1266171 E$
$5.0979656 E$

1.1. 2, 0

$\cos \left(\operatorname{coc}{ }_{1}-6 L_{2}-1 w_{1}-1 w_{2}\right.$

1. 1.0, $c$
$-0.7656198 E-02$
$1.4449888 E 00$

$\begin{array}{ll}1.1 .0, & 1.4449888 E \\ 1.3 .0 & 8.8204293 E-0 \\ 1.1 .1, & 6.4309626 E-0\end{array}$ DC
$-4.8329598 E-01$
$1.0260539 E$ O1
$6.2623650 E$ O0
$5.0161185 E$ OO

$0^{2} \mathrm{c}$

$-3.4749539 E$ OO 7.3132077 OI $4.4552020 E$ OI $4.0807593 E$ OI

C/N

$-1.2116330 E-02$ $7838 E-0$ $1.5796223 E-O$ $1.1517004 E-0$ $4.1775844 E$ OI .888457E 00
DC/N

$-1.1184632 E$ OO $-6.3476110 E$ OO $4.0954468 E$ OI
$\mathrm{C} / \mathrm{M} \times \mathrm{N}$

$-2.2008981 E-0$ -3.0516997e-0L $4.6987743 E 00$
$D^{2} c$ $-2.0612543 E$ OI $0^{2} \mathrm{C}$ 7.7935182E 00 .6364502E 00 $2.1739499 E$
$1.4601922 E$ 02

$0^{2} \mathrm{c}$ 3.2194143E OO $8.4046317 E$
7.63242500

$0^{2} \mathrm{c}$ -2.6212832E 00 -3.9455622E-02 1.7588642E OL $3.0423338 E=0$ $1.1658242 E-01$

1.21142605 OO $4.1372231 E-0$

$1.3690641 E 00$
DCAM
$-1.5840984 E-O$ $6.2171867 E-01$

$-7.5919574 E-02$
C $/$ NeN

$-2.3954967 E-02$ -3.70623604-02 $-1.6209566 E-01$
$9.6346317 E-01$

$1,1,8,0, \cos 1+9 t_{1}-\pi t_{2}-1 w_{1}-1 \omega_{2}$

1. 1,0

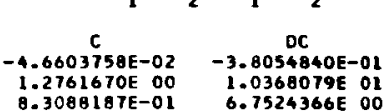

$D^{2} \mathrm{C}$ $-3.1248018 E$ OO $0.4557702 E$ OI $5.5068667 E$ OI $4.1901429 E$ OI
C $1 \mathrm{~N}$

$7.5395979 E-03$ $2.0645944 \mathrm{E}-0$ $1.3442082 \mathrm{E}=01$
$8.4564438 \mathrm{E}-02$ criven

$-1.34030494-02$

1.0096493e-02

$1.1994791 \mathrm{E}-02$ $9.6067095 E-02$

$\begin{array}{lll}1.3 .0, & 0.3088187 E-01 & 6.7524366 E 00 \\ 1.1 .1, & 5.2270965 E-01 & 4.6189906 E 00\end{array}$

DCA $1.4411357 E-0$
$1.6418635 E-00$
$7.0879148 E-0$

$1.1305921 E 00$

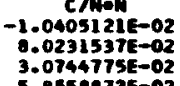

5.074T7SE-02 
$11.1,9,0, \cos 1+10 L_{1}-8 L_{2}-1 H H_{1}-1 H_{2}$

$\begin{array}{ccc}C & C & D C \\ 1.0, & -3.1134893 E-02 & -2.8599477 E-01\end{array}$

13.1.01 $1.0634427 \mathrm{E} \mathrm{O0}$

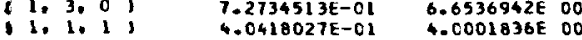

$\mathrm{D}^{2} \mathrm{C}$

$-2.6401119 E 00$ .9269415E O $6.1091353 \mathrm{E}$ OI
$4.0375078 \mathrm{E}$ O1
$C / N$
$-4.5931741 E-03$ $1.5688435 E-01$ $1.0730157 E-01$
$5.9626681 E-02$
$D C / N$

1.2191368E-02 $1.4347521 E$ OO $5.9012697 \mathrm{E}-01$
$\mathrm{C} / \mathrm{N} \in \mathrm{N}$

$0.7760786 E-04$ $2.3144359 E-02$ $1.5829660 E-02$
1. 1. $10 \cdot 01 \cos 1+11 L_{1}-9 L_{2}-1 w_{1}-1 w_{2} 1$

$C$

1. $1.0, \quad-2.0314838 E-02 \quad-2.0728015 E-01$

3.1.0 $8.4666175 \mathrm{E}-01$ 8.6053352E OO

t1.3.0, $6.0209939 E-01$ 6.1208841E OO
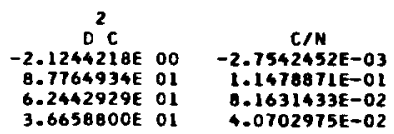

$0.1631433 E-02$
DC/N
$-2.8102629 E-02$
$1.1666942 E$ OO
$0.2985723 E-O 1$ $.2985723 E-01$ $4.4612341 E-01$
$C / N=N$

$3.7341506 E-04$ $1.5562024 E-02$ 1.1067427E-02 c $0,2,-12,01 \cos \left(-12 t_{1}+14 L_{2}+0 w_{1}-2 \mathrm{H}_{2}\right)$

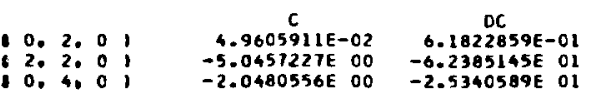

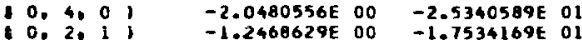

$0.2,-11.01 \cos \left(-11 t_{1}+132_{2}+0 w_{1}-2 w_{2}\right)$

$0,2,0$,

2. 2,0

$0,4,0$

$C$

DC

$424 E-02$

8.0709424E-02 $9.2542536 \mathrm{E}-0$

$-6.8542082 E$ OO $-7.7827044 E$ O

- $1.8311202 \mathrm{E}$ OO

$-3.2178178 E$ OI
$-2.4621231 \mathrm{E}$ ol

$0,2,-1 C, 0, \cos \left(-10 L_{1}+12 L_{2}+O W_{1}-2 W_{2}\right)$

0.2, 0,

2. 2, 0

$0,4,0$

$C$

$1.2996392 \mathrm{E}-01$
$-9.0496774 \mathrm{E}$

$1.3607343 E$ OO

-3.9531040E

$-2.7904684 E$ OD

$-3.3837334 E$
10. 2. $-9.01 \cos 1-9 L_{1}+11 L_{2}+0 w_{1}-2 w_{2}$,

10.2. 0

2, 2, 0

10. 2. 0.1 :

1 $0,2,-8,0, \cos 1-8 L_{1}+10 L_{2}+O H_{1}-2 w_{2}$

10.2 .0

2.2. 2.0

1.0.4. 0,0 :

$C$
$3.2393772 E-01$
$-1.4105833 E$ O1
$-6.3006709 E$ OO

$-5.7727104 E 00$

DC

2.7472190E 00

$-1.1711898 E 02$

$-5.2475890 E$
$-5.8990116 E$
$10.2,-7,0, \cos 1-\pi L_{1}+9 L_{2}+0 w_{1}-2 w_{2}$

10.2.0,

$12,2,0,1$

0. 2. 1

$$
c
$$

$4.9038102 E-01$ $-1.6321542 \mathrm{E}$ OI -7.5953090 OO $-7.9758405 E$ OO

$3.7319002 E$ OO $-1.1863692 E \quad 02$ $-5.5488835 E$ ol
$-7.4041639 E$ ol
(0.2, $-6,0, \cos \left(-6 L_{1}+8 L_{2}+0 w_{1}-2 w_{2}\right)$

1 0.2. 0

12. 2. 0

10,400 :

$C$
$7.4911857 E-01$
$-1.7567674 E$ OI
$-0.4363990 E$ O

$-8.6363990 E$ OO

$-1.0634107 E$ O
OC

$4.8674371 E$ OO $-1.0917747 \mathrm{E}$ O2 $-5.4162242 E$ OL
$-8.8973316 E$ OI
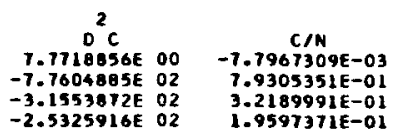

$D C / N$

$-9.7169104 E-02$ $9.8052869 E$ OO 3.9828672E OD
C/NON

1.2254399E-03 $-1.2464693 \mathrm{E}-0$ 5.0594111E-02
2

$1.0721104 E$ OI -3.32574996 O2

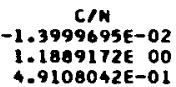
$.9108042 E-01$ $3.2622046 \mathrm{E}-01$

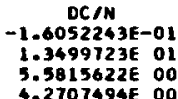

5.
CINON

2.4283589E-03 2.062271 9E-O $-8.5181824 E-02$
2

1.4426127E OI $-4.2579826 E$ O2
$C / N$
$-2.5148936 E-02$
$1.7511764 E 00$ $7.3860139 E-01$ $5.3997530 E-01$
$O C A M$

$-2.6331168 E-01$ 1.8111435E OL T. $6.5477628 E$ OO
C/NAN

4.8664963E-03 $3.3806497 E-0$ $-1.4292457 E-0$
10. 2, $-5.01 \cos 1-5 L_{1}+7 L_{2}+\mathrm{OH}_{1}-2 H_{2}$,

c. $2.0,0$

$2,2,0,0$

1., 2, 1
$1.0922462 E 00$ $-1.7093359 \mathrm{~F}$ O $-9.0832452 \mathrm{E}$ CO
$-1.3545028 \mathrm{E}$ OI

\section{DC}

$6.0186433 E$ OO $-8.7523304 E$ OL $-4.7380792 E$
$-1.013 C 503 E$
$0^{2} \mathrm{C}$

1.88461086 ol -1.0125360 OS OJ $-4.4029759 E$ O2 $-3.2946036 E$ O2 $C / N$
$-4.5228941 E-02$
$2.5255441 E$ OO
$1.0927268 E$ OO
$8.8845658 E-01$ 8.8845658E-01
DC/N

$-4.2853768 E-0$ $2.3553177 E$ O $1.0211353 E$ O $9.9219460 E$ OD
C/N-N

$9.6959358 \mathrm{E}-\mathrm{O}$ -5.5258030E-OI $-1.9439123 E-0$
$0^{2} \mathrm{C}$

2.3757506E 01 -9.0125654E O2 $-4.4178224 E^{02}$
$-6.3641495 E^{02}$ C/N
$-0.1531876 E-02$
$3.5502968 E 00$
1.5858157600
$1.4529333 E 00$
DC/N

$-6.9144747 E-0$ $2.9477673 E$ O $1.3207655 E$ O $1.4847220 E$ OI
C NNAN

2.0520756E-02 $-8.9357408 E-01$ $-3.9913390 E-01$
$-3.6968874 E-O 1$
$D^{2} \mathrm{C}$

$2.8664610 E$ OI -8.6960987 E 02 $-4.1016430 E$ O2 -7.3525821E 02

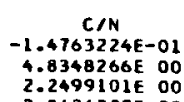

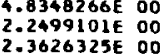

OC/N

$-1.1054770 E$ OO 3.5143060 O OL $1.6431104 E$ O $2.1932684 E$ OI
C/NON

$4.3132159 E-02$ $-1.4321899 E 00$ $-6.6647655 E=01$
$-6.9986759 E-01$
OC/N

$-1.7518133 E$ OO $.9293481 E$ OL $1.9493244 E$ OI
3.2021911 OI
C/NAN

$9.7034349 E-02$ $-2.2755648 E$ OO $-1.1186846 E$
$-1.3774504 E$ 
$0.2,-4.0, \cos \left(-4 L_{1}+6 L_{2}+0 w_{1}-2 w_{2}\right)$

$\because 0.2,0: 1$

$2,2,: 01$

0,40
0,2

1.52777 C

-1.4273670 OI

$-8.5573128 E$ OO

$6.9184366 E$ OO

$-5.6075191 E$ O1

$-3.5237829 E$ OI

-1.6238471E o1 -1.0778419E O2

$1062,-3.01$

$\cos 1-3 x_{1}+5 L_{2}+0 x_{1}-2 w_{2}$

10.2.0.

$2.20: 0$
00,20

2.0123778E 00

$-9.1071465 E 00$

oc

i. 20 1 ! $-2.2004590 E$ OI -1.7903215E O1 -1.0519772E O2
$0^{2} \mathrm{C}$

3.3764417E O1 $-1.9605524 E 02$ $-1.4130793 E$
$-6.284710 E$

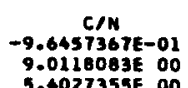

$9.0118083 E$ O0 $1.0252303 E$ OI
DC $/ M$

$-4.3680164 E 00$ 3.54035706 ol 2.2247716E oI
C/NON

$6.0899216 E-01$ $-5.6896853 E$ DO $-3.4110651 E$ OO $-6.4728826 E$ OO
$7.1561522 E$ OO
$0^{2} \mathrm{c}$

2.88584045 OI $-6.5372185 E$ DO $-4.5714908 E$
$-7.5302906 E$
$10.20-2.01 \cos 1-2 L_{1}+4 L_{2}+O w_{1}-2 w_{2}$

10.2001

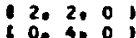

\{.4. 40,

$c$
$2.4106540 E$ 00

$D C$

$-2.8640101 E 00 \quad 0.2750970 E$ OO -3.9874692E OO -5.2519116E OO -1.7471617E OI -9.2036216E O1
$0^{2} \mathrm{c}$

2.0038539E o1 $9.5138611 E$ ol $1.7333950 E$
$-6.3061691 E$ 02
$-2.039769$

9.2317191E 00 1.8146910E OI $6.8995522 E$ OO
DC $/ \mathrm{M}$

$-7.2535602 E 00$ $2.2304119 \mathrm{E}$ ol 2.00781096 OI
$1.0662965 E$ 02
C/NON

2.06753406 OO $-9.3573793 E$ OO $-6.9924338 E$ OO
$-1.8393922 E$ ol
$-10,2,-1,01, \cos 1-11_{1}+3 L_{2}$, ow $-2 w_{1}$

10,2001

2. 20: 0 :

$\left(\begin{array}{lll}0 & 40 & 0\end{array}\right)$

\section{$3.64780708-01$}

oc

oc $2.6341933 E$ OO $1.5465889 E$ OI 0.6498219E-01 $5.5948041 E$ OO
$-1.2291115 E$ O1
$-6.8737137 E$ OI
$D^{2} \mathrm{C}$

$1.0594702 E$ or $4.3214132 E$ o1 $-4.0326579602$
C/N $2.8106701 E$ OO 1.2660897 ol $4.1574210 E$
$-5.9075599 E$
01 oc/m $7.4334724 E$ ol
$2.6890676 E$ oI $2.6890676 E$ oI
$-3.3037584 E$ Oz $1.1012315 E$ ol
C/MeN

1.3509109E O1 6.0052906 EI $1.9982087 E$ ol
$-2.0393095 E$ o2
$10.20-0.01$

10.2 .01

2. 20,

0. 4.00 :

10. 2. 11 $\cos \left(\right.$ tor $\left.x^{+}+2 L^{2}+0 w_{1}-2 w_{2}\right)$

$c$

$1.5281288 E$ OO $1.6536397 E$ OO $2.1573505 E$ OO 1.1803265E OI 7.2473661E-01 $5.5717249 E$ OO
$0^{2} \mathrm{c}$

$0.9757625 E$ OO Q.5679871E OL $3.9007459 E$ OI -3.42719496 o2
C/N $1.8974207 E$ OO $1.8974207 E .00$
$2.6787021 E$
0.00 $0.9987854 E-01$ -1.0714608E O1
DC $M$

$2.0532631 E 00$ 6.92565396 OD $-5.8622704 \mathrm{O}^{\circ}$
CrNoN 2.3559569600 3.326044960 -1.3304179E ol
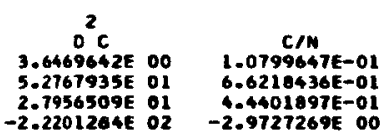

\section{OCA}

$4.0073600 \mathrm{E}-01$ 4.5271450 OOO $2.6610401 E$
$-1.9550790 E$
C/NoN
$7.6992632 E-02$

$4.7200317 E-01$ $3.1654913 E-01$ $-2.1193106 E$ OO

$10.2,2.0, \cos 1+2 \mathrm{~L}, \mathrm{OL}$, tow, $-2 w^{\prime}$

10. 2.0

$12020: 9$

$$
c
$$$$
\text { DC }
$$

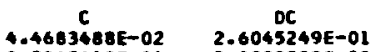
$3.2345419 E-01 \quad 2.8233533 E$ OO 2.7655529E-01 2.0013807E OO

1 $0.2,1$,

$-1.0300106 E$ OO $1.4418843 E$ o1
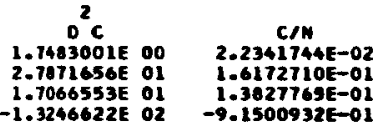

DCAM $1.3022624 E-01$ $1.4116767 E$ OO

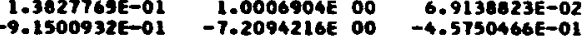

C/NON

1.1170872E-02 ..0863547E-02

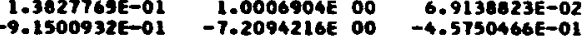

$10,2,3,0, \cos 1+3 L_{1}-1 L_{2}+0 w_{1}-2 w_{2}$

$10.2,0$

2. 20:

i: $0.40: 1$ :

$10.2,4.0$

c $351 E-02$

DC $2.4341211 E-02$ B.8407848E-01 $1.1265299 E-01$ 9.6089402E-01

$\cos 1+4 l_{1}-2 L_{2}+w_{1}-2 w_{2}$

10.2. 0

$2,2,0$

:0: 2,01 i

0.0075710

c -6.7995210E-01 2.5910830E-01

$10,2,5,0$,

$\left.\cos 1.5 L_{1}-3 L_{2}+0 w_{1}-2 w_{2}\right)$

$10.2,0$

2,200

i.: 2,1 ;

c

$-4.7109609 E-01$

$-3.9120721 E-02$

DC

$1.5313876 E-01$ - $2503061 E 00$

$-2.3641828 E$ OO
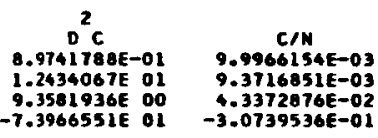

OC/M

$5.1847255 E-02$ $3.4038100 E-0$ $-2.7614031 E$ OO

CAMen

$3.84062796-03$ $3.6082216 E-03$ $1.6699126 \mathrm{E}-02$ $-3.0739536 E-01$

$0^{2} \mathrm{C}$

$7.3925601 E-01$ $1.2867606 \mathrm{E}$ OO $4.4683306 E$ OO $-4.0631331 E$ O1
$\mathrm{C} / \mathrm{M}$

$0.9762269 E-03$ $7.5226445 E-02$ . $-1.2995312 E-01$
OCA

$0.2166003 E-02$ 2.1204232E-O $1.1241004 E-01$
$-1.1726373 E$ c/mon

2.8097875E-03 $-2.3547792 E-02$ $2.5221949 \mathrm{E}-03$
$D^{2} \mathrm{C}$

$8.5583770 E-01$ $-9.9727751 E$ DO -2.4296127E o1
TC/N

$-1.6302865 E-03$ $-1.0316802 E-02$ $-1.0316802 E-02$
$-7.5327158 E-02$
$D C / N$

$4.0385306 E-02$

$-6.0135364 E-01$

$-2.5164971 \mathrm{E}-0$ 
$(0,2,6, C) \cos \left(+6 L_{1}-4 L_{2}+O w_{1}-2 H_{2}\right)$

$\begin{array}{llc}\text { 10. 2. } 01 & \text { C } & \text { DC } \\ 0.5370464 E-02 & 1.5701429 E-01\end{array}$

12, 2.0 0 -6.2595080E-01 $-3.7690972 E$ OO

$\begin{array}{lll}0,4,0, & -8.8666059 E-02 & -4.9845531 E-01 \\ 10,2,1, & -2.3620691 E-01 & -1.8811549 E\end{array}$

${ }^{2} C$
$9.9252933 E-01$
$-2.2386210 E$ OI

$-2.4503961 E$ DO
$-1.7648328 E$ OI

C/N

$5.7801275 E-03$ $-1.4260975 E-01$

$-5.3814785 E-02$
DC/N

3. $5772410 E-02$ -8.5870966E-01 $-1.1356258 \mathrm{E}-01$

$-4.2858164 E-01$
C $M=N$

1.3168807E-03 $-3.2490636 E-02$ $-4.6023052 E-03$ $-1.2260569 E-02$

t0.2, $7,0, \cos 1 * 7 L_{1}-5 L_{2}+0 w_{1}-2 w_{2}$,

C DC

10.2.0, $\quad 2.0144569 \mathrm{E}-02 \quad 1.4438954 \mathrm{E}-01$

2. 2. $01, \quad-6.8942924 E-01 \quad-4.8824249 E 00$

$\begin{array}{lll}0,40,1,1, & -1.1944622 \mathrm{E}-01 & -8.3221906 \mathrm{E}-01 \\ 0,2,1 & -2.0353463 \mathrm{E}-01 & -1.6955636 \mathrm{E} 00\end{array}$

$D^{2}$

$1.0462289 E 00$ $-3.4572053 E$ OI -5.6796002E OO
$C / N$
$4.0397637 E-03$

$-1.3825718 \mathrm{BE}-0$

$-2.3953576 E-02$

$-4.0816550 E-02$
OC/N

$2.8955678 E-02$ $-9.7911468 \mathrm{EE}-01$ $-1.6089205 E-01$
$-3.4002595 E-01$
C/MEN

$0.1012858 E-04$ $-2.7725902 E-02$ $-4.8036172 E-03$
$-6.1852942 E-03$
$10.2,6.01 \cos 1+8 L_{1}-6 L_{2}+0 x_{1}-2 H_{2}$,

$\begin{array}{ccc}0.2,0, & C & \text { DC } \\ 2.4940920 E-02 & 1.2209661 E-01\end{array}$

2.2.0, $20.7446350 E-01 \quad-5.4727383 E$ OO

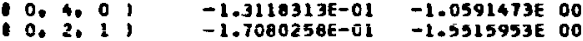

10.2. $0.01 \quad \cos 1+9 L_{1}-7 L_{2}$ on $-2 w_{2}$

0.2. 0

2. 2. 0

$C$
$1.0556813 \mathrm{aE}-02$
$-6.0699980 E-01$
$-1.2763028 \mathrm{~B}-01$

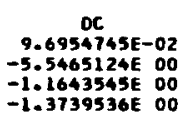

10.2. 10.0

$\cos \left(+10 \mathrm{~L}_{1}-8 L_{2}\right.$ on $\left.{ }_{1}-2 \mathrm{w}_{2}\right)$

10. 2, 0

2, 2, 0

C

C $297 E-03$

$-5.1325400 E-0$

$-1.1429704 \mathrm{E}-0$
$-1.0573260 \mathrm{E}-0$.

DC

$7322 E-02$

130799E OO

$-1.1600991 E$
$-1.1638776 E$

$1-1 \cdot 1,-10 \cdot 0) \cos \left(-111_{1}+11 L_{2}+1 w_{1}-1 w_{2}\right)$

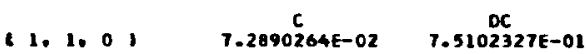

$\left.1-1,1,-9,01 \cos 1-10 \mathrm{~L}_{1}+10 \mathrm{~L}_{2}+1 \mathrm{w}_{1}-1 \mathrm{w}_{2}\right)$

2

${ }^{2} \mathrm{C}$

T.5192025E-0I

$-5.3121503 E$ OI

$-1.1005956 E$ OI

$-1.3092221 E$ ol

1.0615645E-03 $-7.5717791 \mathrm{E}-02$ $-1.6861784 E-0$ $-1.5594199 \mathrm{E}-02$

$1.0826463 E-02$ $-7.6905943 E-01$ $-1.71143586-01$ $-1.1114358 E-01$
$-1.7170101 E-01$

C/NOH

1.5660727E-04

$-1170265 E-02$

$-2.4975342 E-03$

$-2.3011237 E-03$
$0^{2}$

7.7921373E 00

C/N

$3638 E-02$

oCs

$30306 E-01$
$.6084122 E-03$
$\begin{array}{cc}C & D C \\ 1.1380450 E-01 & 1.0571188 E\end{array}$

$C / N$

9.8970086 E 00

2

D C
$1.2025411 E$ OL

$-3.2418135$

$35 E-02$

DC 1

C7N

CNNON
$6.0303522 E-03$

1. 1. $-7.01 \cos \left(-8 e_{1}+8 L_{2}+1 x_{1}-1 x_{2}\right)$

$\begin{array}{ccc}\text { 1 } 1.1 .0, & \text { OC } \\ 2.0035515 E-01 & 1.0864701 E & 00\end{array}$

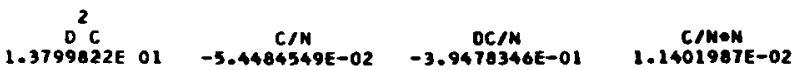

$1-1.1,-4.01 \cos 1-7 L_{1}+7 L_{2}+1 H_{1}-1 w_{2} 1$

C $D C$

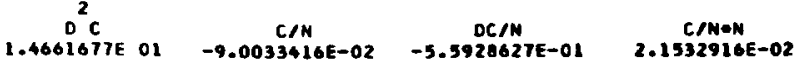

1-1.1. - -5. 0, $\cos 1-6 L, 6 L, 1 w-1 \%$,

$\begin{array}{ccc}\text { 1. } 1.01 & \text { CC } \\ 5.2021945 E-01 & 2.6056150 E & 00\end{array}$

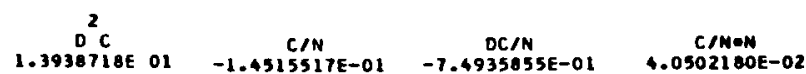

$1-1.1,-4.01 \cos 1-5 t_{1}+5 L_{2}+1 w-1 w_{2}$

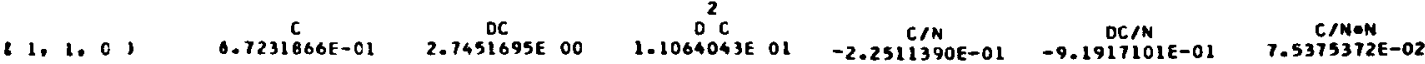


$1-1,1,-3,0, \cos \left(-4 L_{1}+4 L_{2}+1 \omega_{1}-1 \omega_{2}^{\prime}\right.$

1.1.0, T.to639ioe $\rightarrow 01 \quad 2.2857480 E$

t-1. 1. -2, $0, \cos \left(-3 t_{1}+3 L_{2}+1 w_{1}-2 w_{2}\right)$

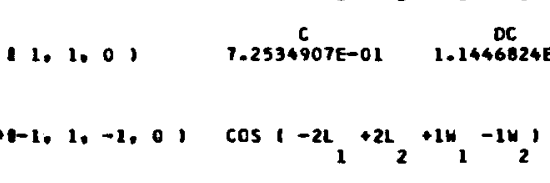

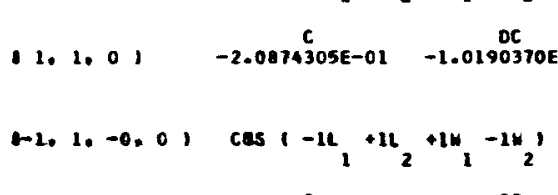

$\begin{array}{lll}1.1 .01 & C & \text { DC } \\ C & -4.3460676 E-01 & -1.5688525 E\end{array}$

$0-1,1.2,0, \cos 1+\mathrm{OL}_{1}+\mathrm{OL}_{2}+1 \mathrm{H}_{1}-\mathrm{IW}_{2}$

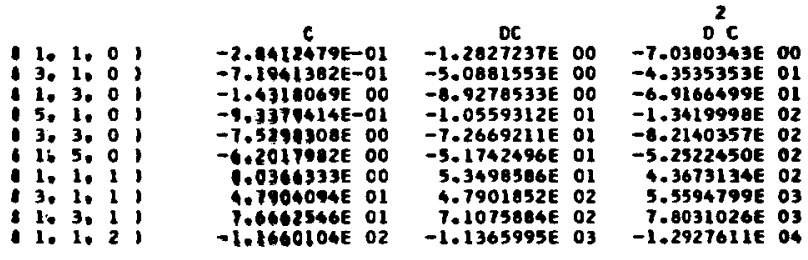

$1-161,2,0, \cos 1+1 L_{1}-1 L_{2}+1 u_{1}-1 w_{2}$

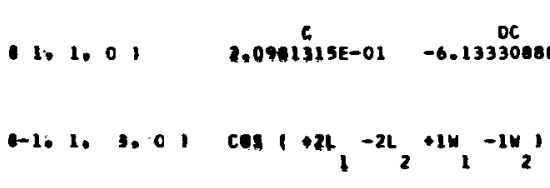

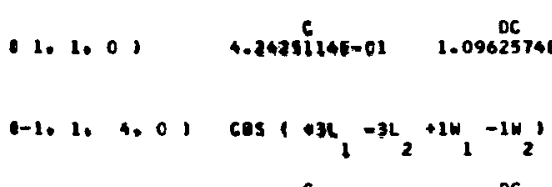

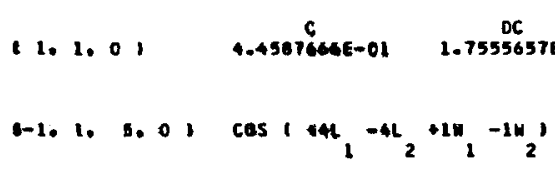

$1,10, \quad$ DC

$1-1.10,0, \cos 1+x_{1}-5 L_{2}+1 w_{1}-1 w_{2}$

1. 1. $0, \quad 2.9262567 E-01 \quad 1.8033388 E 00$

$\left.-1.101 .0, \cos 1+\omega_{1}-6 L_{2}+1 H_{1}-1 H_{2}\right)$

$\begin{array}{ccc}\text { 1. } 1.01 & C & \text { DC } \\ 2.1073991 E-01 & 1.5198760 E 00\end{array}$

1. 1. $1.0, \cos \left(\pi x_{1}-7 L_{2}+1 w_{1}-1 w_{2}^{\prime}\right.$

1.1 .01
$1.4524007 E-01$
$1.1 .1976326 E$

$\begin{array}{ccc}C & C & \text { DC } \\ 1.0, & 9.6960071 E-02 & 0.9805844 E-01\end{array}$
$0^{2} \mathrm{c}$

5.9973701E 00

$-3.2580805$

DCIN

C/MUK
$-9.3667740 E-01$
$1.3636376 E-01$

$-20$

$-2.4681659 E-01$

$C / N$
$-4.0478365 E-01$

$\stackrel{D C / N}{-6.3079412 E-01}$

C/NeN
$2.2589097 E-01$

$0^{2} c$

$D C$
$-6.0144583 E$

$\underset{1.7473471 E-01}{C / N}$

OC/ $1 \mathrm{M}$

6.5301590E-01

CIMEN
$-1.4626700 E-01$

$D^{2} c$
$-7.6761151 E 00$

C/N

DCN

$N$

Conew
$-1.2181220 E$ oo

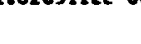


$3-1,1,10.0, \cos \left(+9 L_{1}-9 L_{2}+1 H_{1}-1 H_{2}^{\prime}\right.$

$\begin{array}{lll}1.1 .01 & C & D C \\ & 6.3183604 E-02 & 6.5009517 E-01\end{array}$

$10,0,-7,1, \cos \left(-6 L_{1}+8 L_{2}+O w_{1}+O w_{2}{ }^{\prime}\right.$

$0,0,1$

$2,0,1$

$0,0,2$;

C
$4.3413610 \mathrm{E}-02$
$-4.6444061 \mathrm{E}-01$
$-4.6800217 \mathrm{E} 00$

DC
$4.0693702 E-01$ $-4.6444061 E-01 \quad-3.3393811 E$ OO

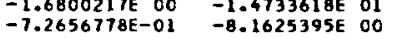

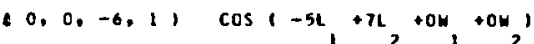

$0,0,1$

2.0.1 1

$\begin{array}{lll}1 & 0.2,2 & 1 \\ 1 & 0.0 .2 & 0\end{array}$

$C$
$7.4672676 E-02$
$-3.2219307 E-01$

$-1.6117281 \mathrm{E}$ ol

$-1.1130446 E$ OD $-1.1493813 E$ OI

( $0, c,-5,1) \cos (-4 L, 6 L,+0 w,+0 w$,

0.0 .1

2., 0,1$$
c
$$

C $560 E-01$

$1.2723560 E-O 1$
$7.3051466 E-02$

$-2.4716606 E \mathrm{C}$

\begin{abstract}
9. $4208562 \mathrm{E}-01$
$3.0760530 E$ OO

$-1.5764860 E$ OI
$-1.5721527 E$ OI
\end{abstract}

$D C$

$0,0,2$

$-1.6666411 E 00$

10.0.-4.1)

$\cos 1-3 L_{1}+5 L_{2}+O w_{1}+O w_{2} 1$

$0,0,1$

$2,0,1$,

, $0.0,2$,

$$
2.1405162
$$

C $162 E-01$

$8.5016803 \mathrm{E}-01$

$-2.5749180 E$ OO

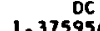

OC $9568 E 00$

$9.3746370 E$ OO

$-1.2640672 E$ OI

$0,0.1$

2, 0,1

$c$

C $759 E-01$

$3.5367759 E-01$
$2.0807919 E$ OO

$-2.1633392 \mathrm{E} O 0$

DC

OC

$10,0$.

$-3.3638519 E O O$

D.

$-5.9266175 E$ OO

$+0,0,-2,1, \cos 1-1 L_{1}+3 L_{2}+O w_{1}+0 w_{2}^{\prime}$

C.0. 1

2. 0,11

1.0.2, 0.2

$$
5.6824959
$$

C $959 \mathrm{E}-0$

$3.5921294 E$ OO

$-9.5386733 E-C 1$

$-4.4106728 E$ OO

2.5654474

$2.5654474 E$ OO

$2.4825200 E$ O

$4.3016289 E$
$-3.1389979 E$

$40,0,-1,1$,

$\cos 1+\mathrm{OL}_{1}+2 \mathrm{~L}_{2}+\mathrm{OH}_{1}+\mathrm{OH}_{2} \mathrm{H}^{\prime}$

t $0,0,11$

$20,2,1$

0.6921352

C $352 E-01$

$4.6224838 E$ OC

1. $1456297 E$ OO

$-5.2940982 E \mathrm{CO}$

$3.1377049 E 00$

$1.6172306 E$ OI

$-3.5159796 \mathrm{E}$ OI
$0^{2} \mathrm{C}$

$6.1318786 E 00$

$C / N$
$1.1753279 E-02$

$D C / N$
$1.2092931 E-01$

$C / N=N$

$2.1863190 E-03$
$0^{2} \mathrm{c}$

$3.9868605 E 00$ $-1.9252317 E$ O $-1.3008441 E$
$-9.7922919 E$

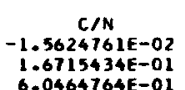
$1.6715434 E-01$
$0.0464764 E-01$ $2.6149513 E-01$
DC/N
$-1.4645853 E-01$
$1.2018588 E$ OD 5.3026977E 0O 2.9377360 EO
C/NON

$3.6234242 E-03$ $-6.0159626 E-02$ $-2.1761550 E-01$ $-9.4113317 E-02$ to. $0 .-3,1, \cos 1-2 L_{1}+4 L_{2}$, ow tow

2. 0,1

$2.8723126 E$ O

$0^{2} \mathrm{C}$

$7.4931969 E$ OO $5.3160612 E$ OI $.6703365 E$ OI $-1.6329693 E 02$
2

$9.7309338 E 00$

$1.0667448 E$ O2 $-2.9020411 E$ O

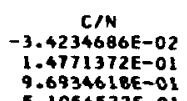

$9.6934618 E-01$
$5.1056522 E-01$

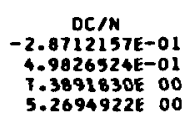

C/NAN

1.5695349E-02 $-6.7721326 E-02$ $-4.4440970 E-01$
$-2.3407544 E-01$ $\left.-10.0 .-c .11 \cos 1+1 t_{1}+1 L_{2}+0 n_{1}+0 w_{2}\right)$

1 0.0.1

$2,0,1$

$40,0,2,1$

$$
6.4363134 E
$$

$0.4363134 \mathrm{E}-01$

$3.8385634 E$ OO

$-5.5471720 E$ OO

$20 \mathrm{C}$

. $5297341 E$ OO

2.597088E OI

$-3.6520874 E$ O
$10,0,1,1, \cos 1+2 L_{1}+\mathrm{OL}_{2}+\mathrm{OH}+\mathrm{O} \mathrm{w}_{2}$,

$0.0,1$

$0,2,1$

0.09213

$352 \mathrm{E}-01$

OC

$4.6224838 E$
$-5.2940982 E$

$-3.5159796 E$ OI

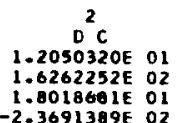

C/N
$-9.0859706 E-01$
$-5.3455505 E$ OD
$5.5576143 E$ OO
$8.6417291 E 00$

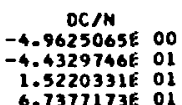

C/NON

C.3341842E OO $2.3341842 E$
$1.3732709 E$ $1.4277501 E$ OI
$0^{2} \mathrm{C}$

$1.4076069 E$ ol 2.0780862E O2 9.5200070 E

$-2.6769411 E$ O2
1.5352230E O1 $2.2782698 E$ O2

1.6641806 E 02

C/N
$1.0792701 E 00$ 5.7395664E DO $1.4224858 E$ OO $1.4224858 E$
$-6.5734851 E$
OC/N $3.0959717 E 00$ 386 O1 (1)
C / N $=N$ 1.7662470E OO -8.1620523E OD
.
$0^{2} \mathrm{C}$

1.5099645E O

2.1433487E O2

$-2.9584625 E$ O2
$C / N$

4.7365BOE-O -3.9546700E OO 2.7365BLE OO

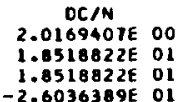

$C / N \circ N$

$3.2712699 \mathrm{E}-01$

$1.9509580 E$ OO

$1.9509580 E$ OO
2

1.53522306 o1

$1.6641806 E$ O2

$2.2782698 E 02$
$-2.8841708 E 02$
$C / N$

$3.3460676 E-01$ $5.7281484 E-01$ -2.3112419E OO
$D C / N$

1.508B525E OO 1.4361563E 01

-1.7579898 ol
$C / N=N$

$2.1730338 E-01$ $1.1556209 E 00$ 


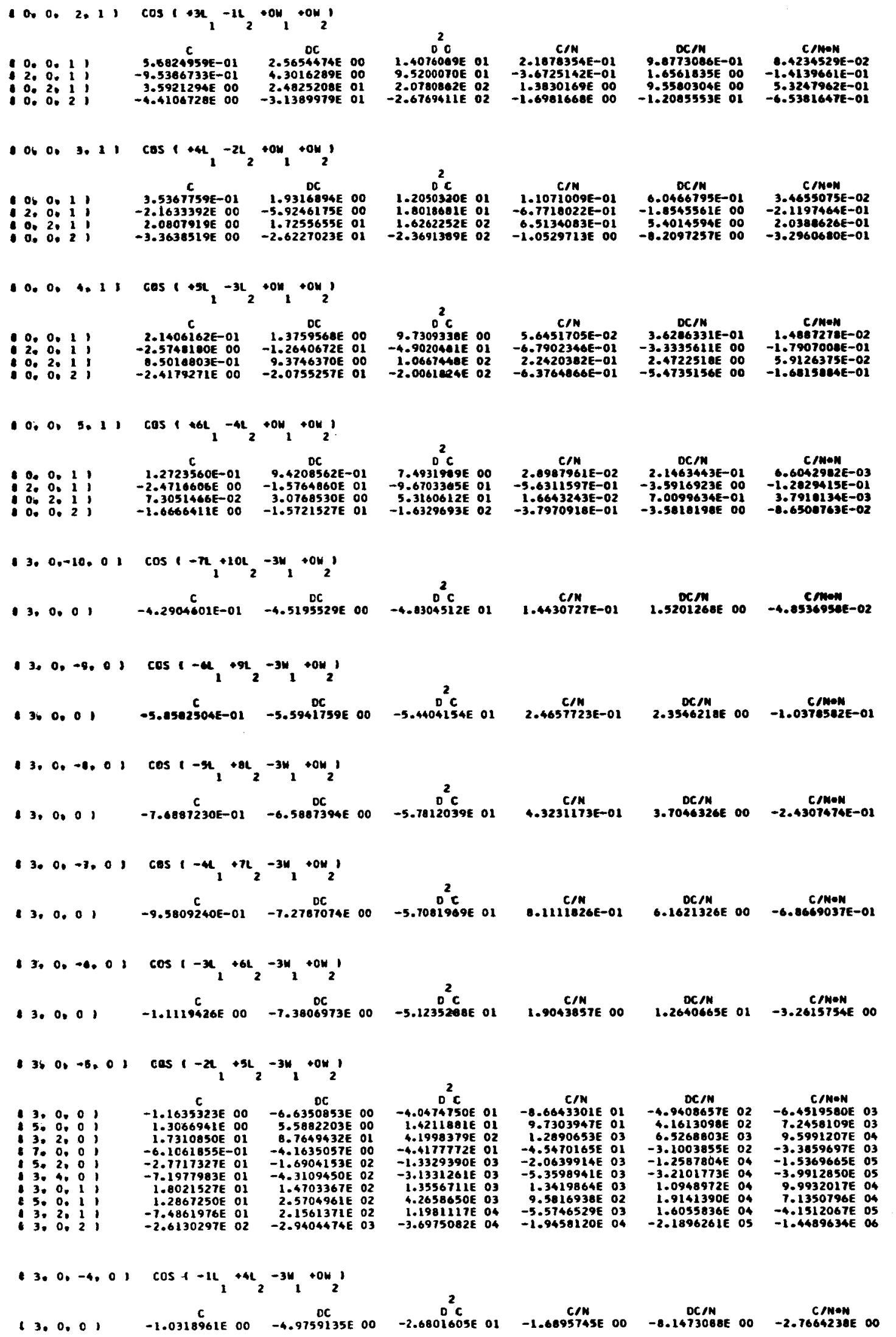




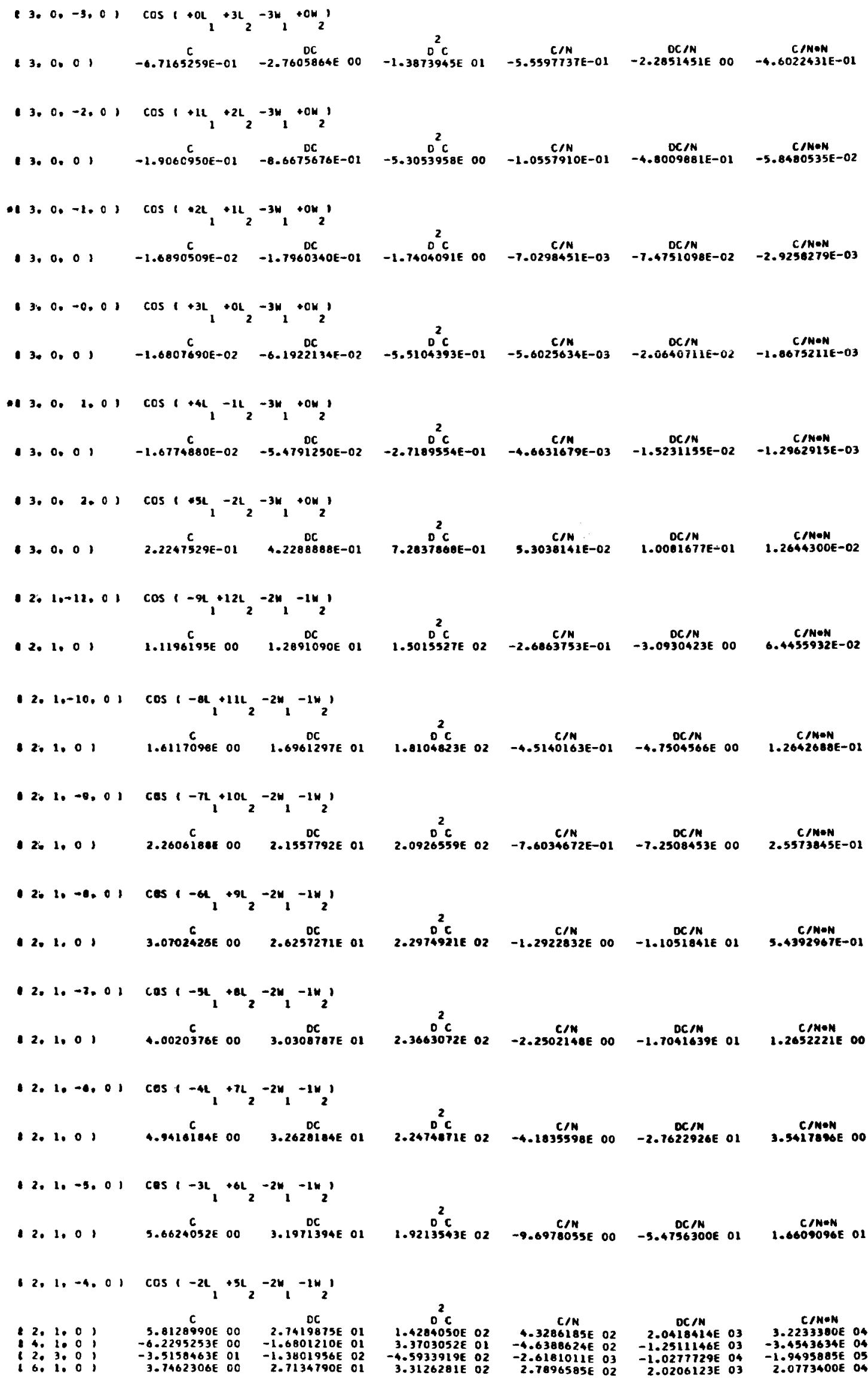




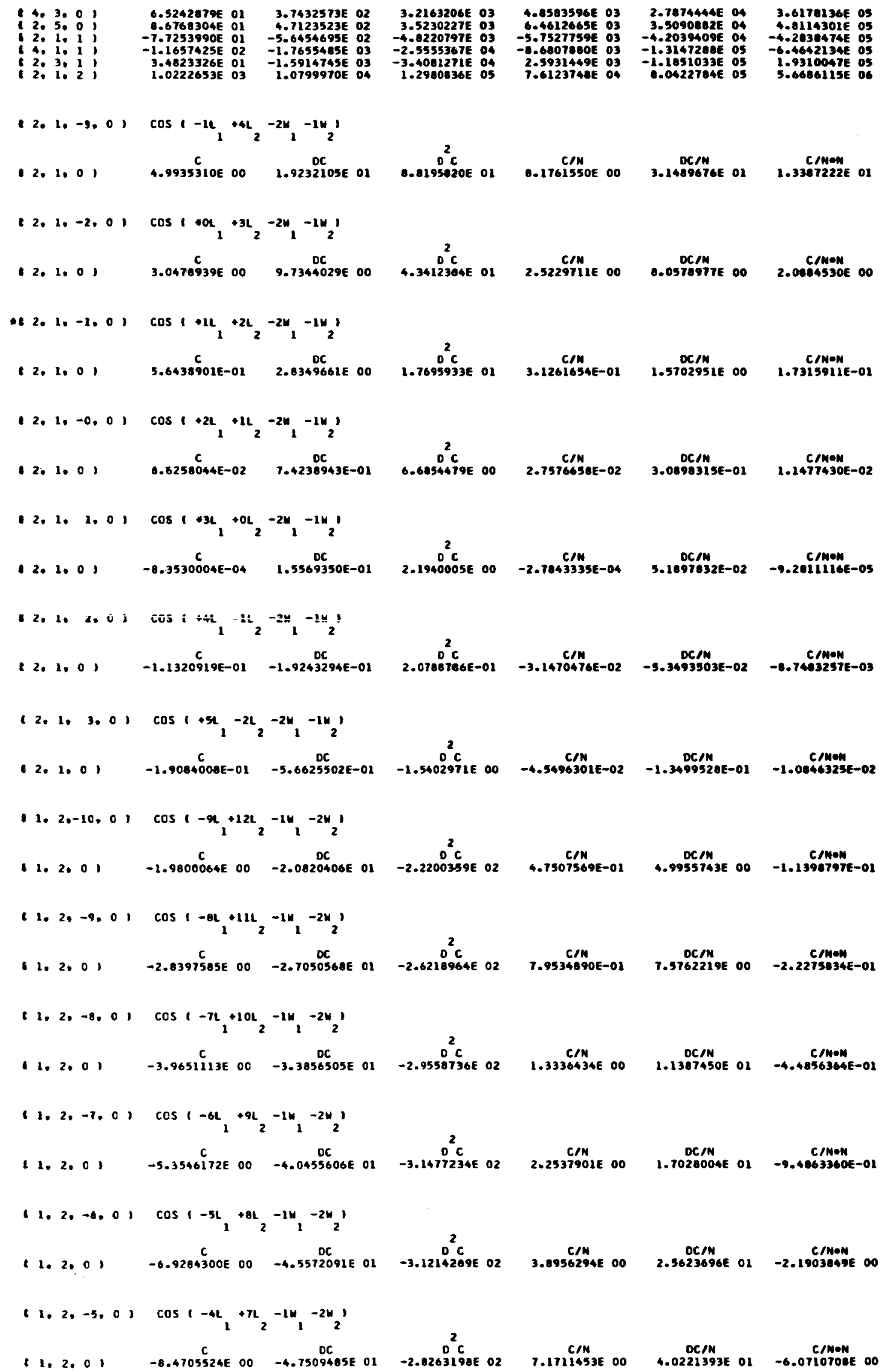


1. $2,-4,0, \cos \left(-3 L_{1}+6 L_{2}-1 H_{1}-2 w_{2}\right)$

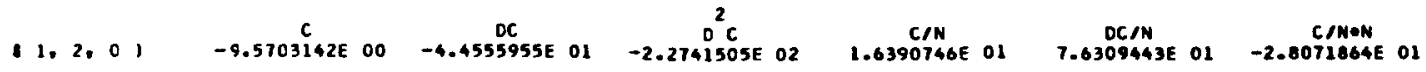

1 $1,2,-3,0, \cos \left(-2 L_{1}+5 L_{2}-1 H_{1}-2 W_{2}\right)$

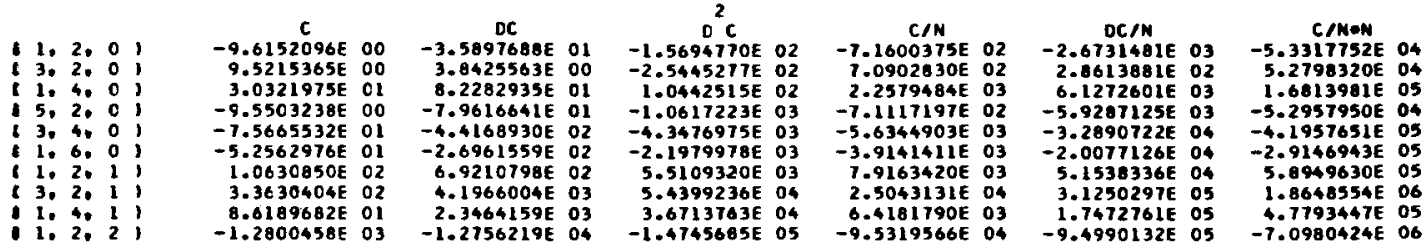

1. $\left.2,-2,0, \cos 1-1 L_{1}+4 L_{2}-1 H_{1}-2 w_{2}\right)$

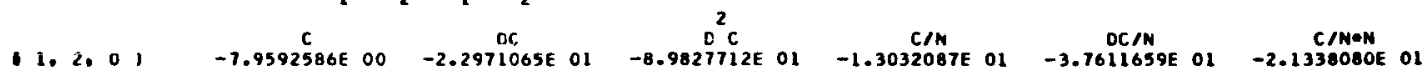

-1. $1,-1,0) \cos \left(+0 L_{1}+3 L_{2}-1 w_{1}-2 w_{2}\right)$

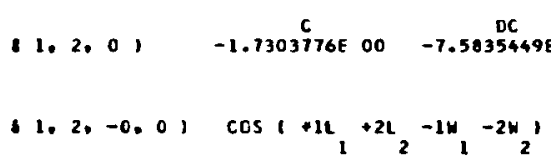

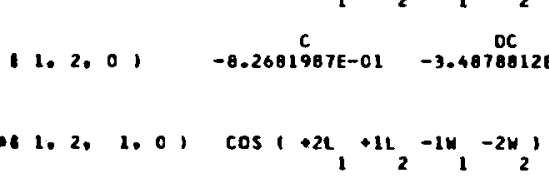

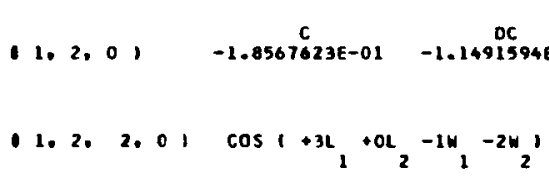

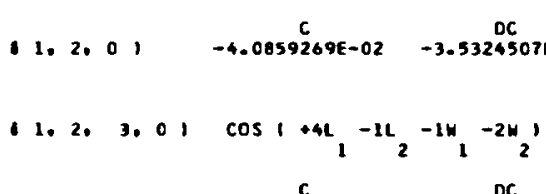

1.2.0,
1. $1.2,4.0, \cos \left(+5 L_{1}-2 L_{2}-1 \%,-2 w_{2}\right)$

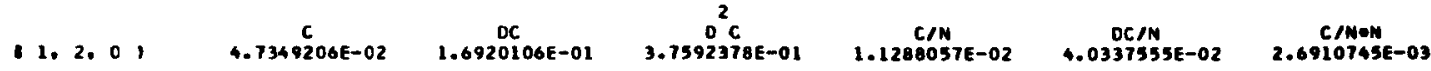

6. $0 .-10,0, \cos 1-10 L_{1}+13 L_{2}+0 N_{1}-3 H_{2}$,

$10.3 .01 \quad 7.9798868 E-01 \quad 0.3843396 E$

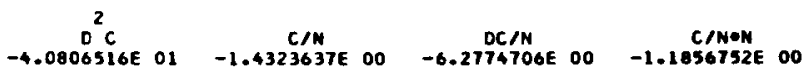

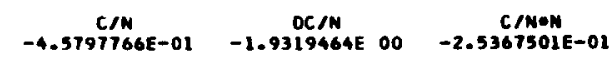

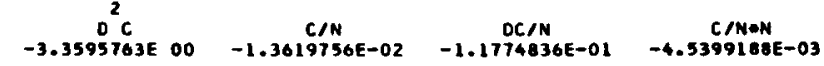

$0^{2} \mathrm{c}$

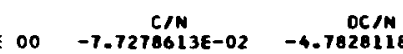

C/NON
BE-01 $-3.2163428 E-02$

10.3. $-9.0, \cos 1-9 \mathrm{~L}_{1}+12 \mathrm{~L}$, ow $-3 w_{2}$,

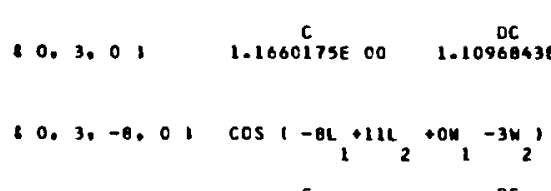

$\begin{array}{lll}10.3 .0, & 1.6657826 E 00 & 1.4205106 E\end{array}$

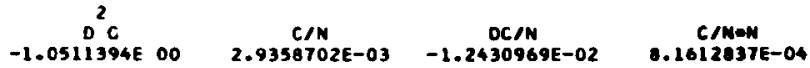

$2 \mathrm{C}$

C/N DC/N

10. 3. $-7,0, \cos 1-7 c_{1}+10 L_{2}+0 w_{1}-3 w_{2}$,

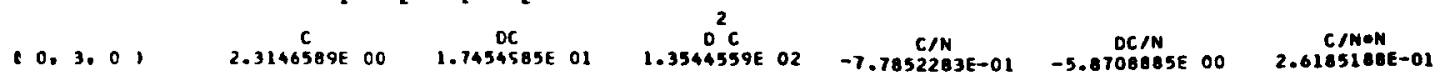




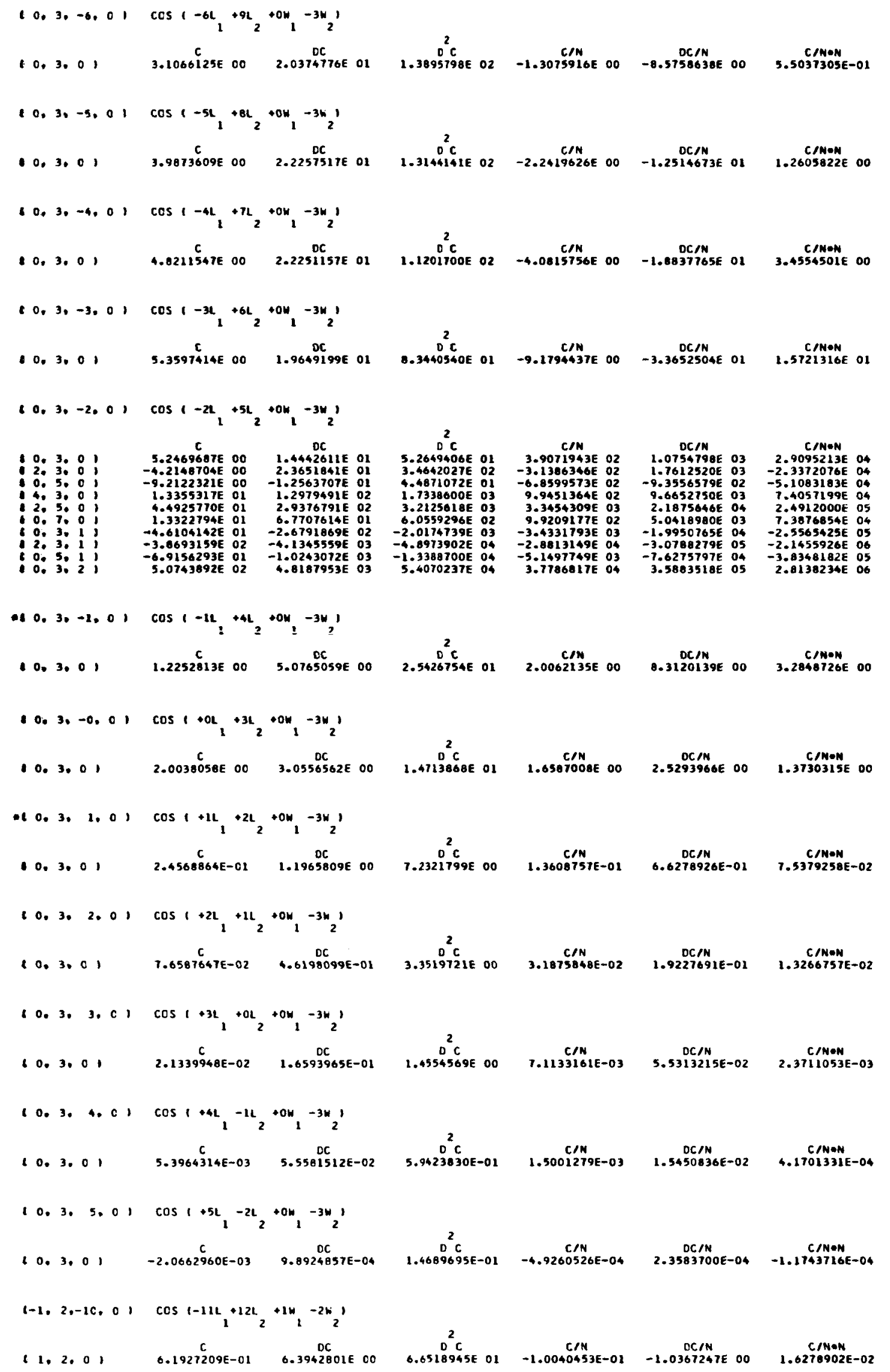


$\left.1-1,2,-9,01 \cos 1-10 L_{1}+11 L_{2}+1 \omega_{1}-2 w_{2}\right)$

$11.2 .01 \quad 8.0114573 E-01 \quad 8.2044603 E$

$\left.1-1,2,-8,0, \operatorname{ccs} 1-9 L_{1}+10 L_{2}+1 w_{1}-2 w_{2}\right)$

$\begin{array}{llc}1,2,01 & 1.2178923 E \text { DO } & 1.0098999 E \text { Ol }\end{array}$

$1-1,2,-7.01 \cos 1-8 L+9 L_{2}+1 w_{1}-2 w_{2}$

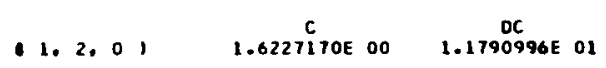

$1-1,2,-6,0) \cos \left(-12+8 L_{2}+1 w_{1}-2 w_{2}\right)$

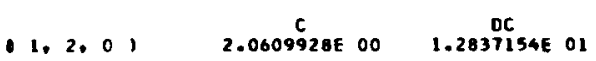

$\left.1-1,2,-5,0, \cos 1-6 L_{1}+7 L_{2}+1 x_{2}-2 w_{2}\right)$

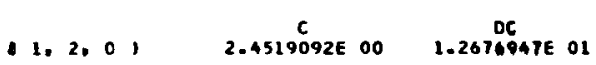

$-1,2,-4,0, \quad \cos 1-5 t_{1}+6 L_{2}+1 w_{1}-2 w_{2}$

1. $2.01 \quad 2.6518776 E_{0} 00 \quad 1.01915370_{01}$

$0-1,2,-3,0, \cos 1-4 L_{1}+5 L_{2}+1 W_{1}-2 W_{2}$

$\begin{array}{lll}1.20 & C & \text { DC } \\ 2.4590575 E & 00 \quad 1.0392245 E & 00\end{array}$

$1-1.2,-2,01 \cos 1-3 L_{1}+4 L_{2}+1 w_{1}-2 w_{2}{ }^{\prime}$

1. 2.01 1.6833007E $00 \quad 2.1325262 E$ $C$
$1.6033007 E$ oo
$\left.\cos 1-2 L_{1}+3 L_{2}+1 W_{1}-2 W_{2}\right)$

$-t-1,2,-1,0) \cos \left(-2 L_{1}+3 L_{2}+1 H_{1}-2 W_{2}\right)$

$1.20,0, \quad-5.6001614 E-01 \quad-3.0011575 E$ DO $0^{2} \mathrm{C}$

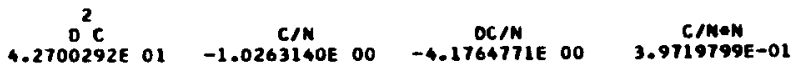
$0^{2} \mathrm{C}$ $8.6532201 E$ OI

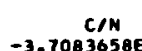<smiles>[Mg][Mg]</smiles>
$D C / N$ $-3.7083658 E-01 \quad-2.6945750 E$ OO 6.4746611E-02

$0^{2} \mathrm{C} C$
$0.06765717_{01}$

$0^{2} \mathrm{c}$ $6.56934110^{\circ}$ O1

$-7.70749$ C/N

oc/

OC/N

\section{$0^{2} \mathrm{c}$} $1.6203061 E$ O2
$-1.23704025$ C/N
oC/ $/ \mathrm{M}$

$34044 E$ DO
C/NON $6.2310392 E-01$

$1-1,2,-0,0, \cos 1-1 L_{1}+2 L_{2}+1 H_{1}-2 w_{2}$

.1. $2.01 \quad-8.2681987 E-01 \quad-3.4878812 E$ $02 \mathrm{c}$

$-1.2116988 E$ CON

OC/N

$0.1219206 E-01$

11-1. 2. 1, $0, \cos 1 \cdot 0,+1 L_{2}+1 w_{1}-2 w_{2}$

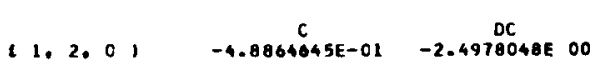

$0^{2} \mathrm{C}$

2 $2 E$ OI

C/N

3.7896149E

$-8.9419675 E-01$

$\left.1-1,2,2,0, \cos 1.1 L_{1}+O L_{2}+1 W_{1}-2 W_{2}\right)$

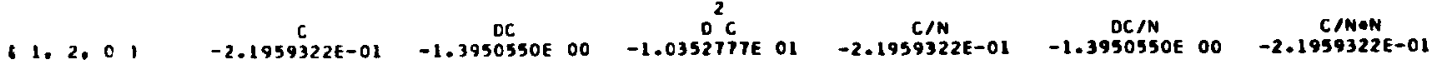

1-1. 2. 3. $\left.01 \cos 1+2 L_{1}-1 L_{2}+1 w_{1}-2 W_{2}\right)$

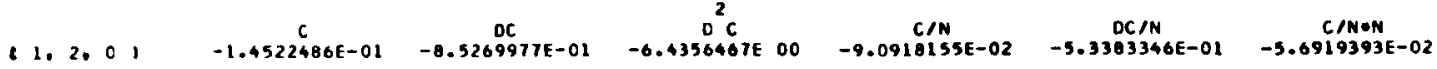

$\left.1-1,2,4,0, \cos 1+3 t_{1}-2 t_{2}+1 x_{1}-2 w_{2}\right)$

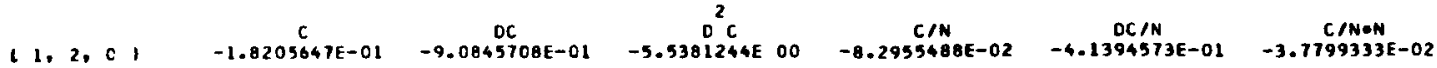




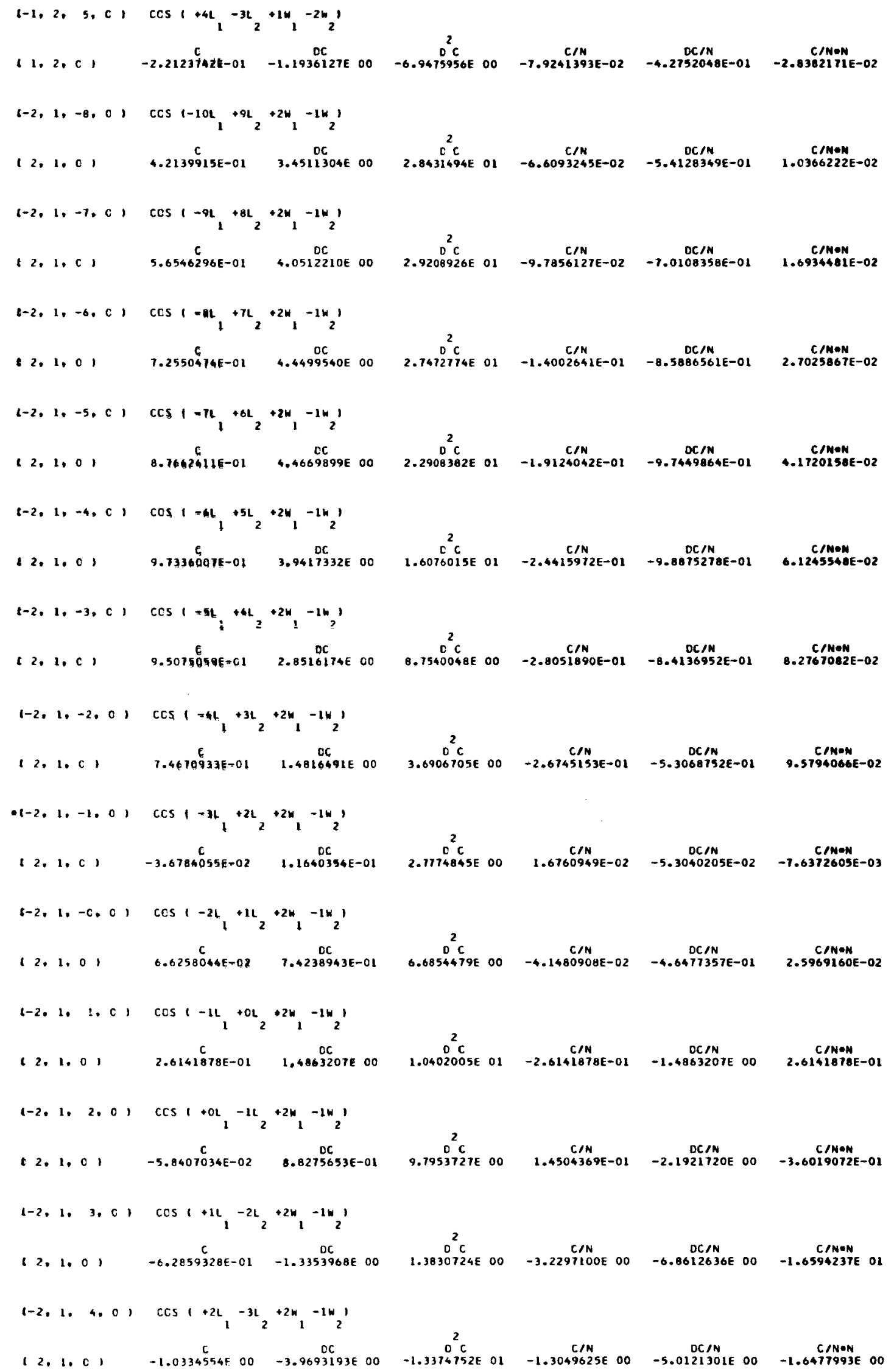


1-2. 1. 5. C $1 \cos 1+3 L_{1}-4 L_{2}+2 H_{1}-1 H_{2}^{\prime}$

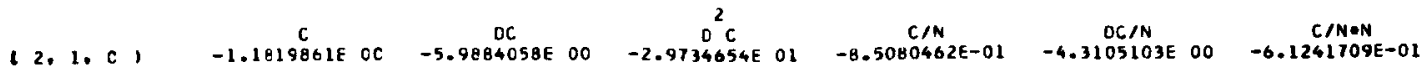

1-2. 1. 6. C, $\cos t+4 L_{1}-5 L_{2}+2 w_{1}-1 k_{2}$,

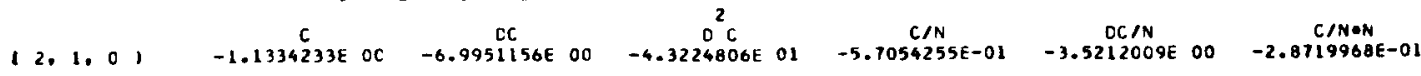

1-2. 1. 7. C 1 $\operatorname{ccs} 1+5 L_{1}-6 L_{2}+2 w_{1}-\ln { }_{2}$

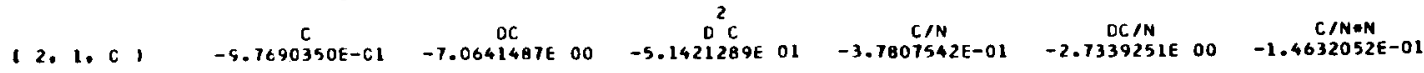

1-2. 1. 8, C, $\cos 1+6 L_{1}-7 L_{2}+2 w_{1}-1 h_{2}{ }^{\prime}$

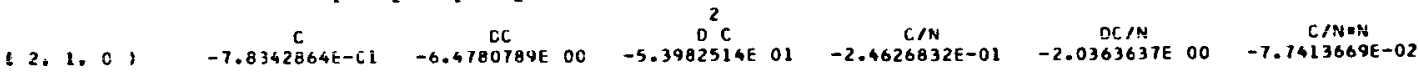

$1-2,1.5,01 \cos 1+7 L_{1}-8 L_{2}+2 w_{1}-1 w_{2} 1$

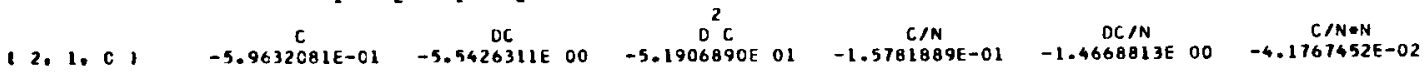

$1-2,1,1 c, 0, \cos 1+\theta L_{1}-9 L_{2}+2 w_{1}-1 k_{2}{ }^{\prime}$

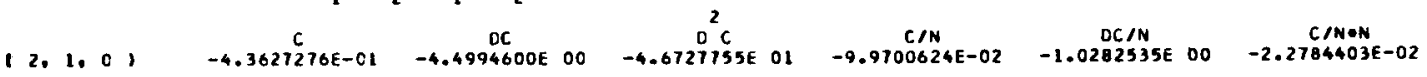

1. $0,-7,1, \cos 1-5 \mathrm{~L},+8 \mathrm{~L},-1 \mathrm{w},+0 \mathrm{H}_{2}$

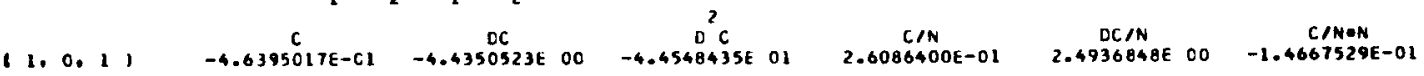

1. $\left.0,-4,1, \cos 1-4 L_{1}+7 L_{2}-1 w_{1}+0 w_{2}\right)$

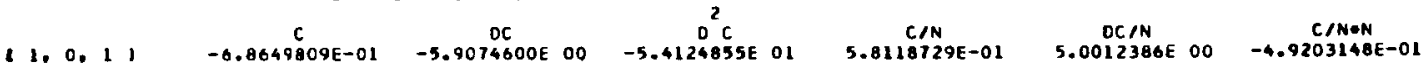

11. $0,-5,11 \cos 1-3 L_{1}+6 L_{2}-1 w_{1}+\mathrm{OH}_{2}$,

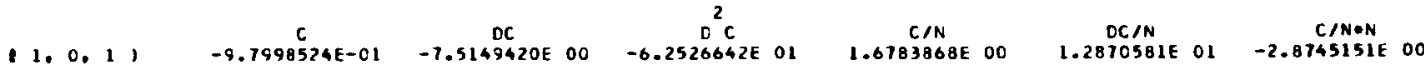

1. $0,-4,1, \cos 1-2 L_{1}+5 L_{2}-1 w_{1}+0 w_{2}$,

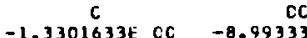

1. 0. 1 - $1.0423519 E$ OO $-4.1940691 E$ OD

3. 0. 1 - $1.0423519 E$ OO $-4.1940691 E$ OI

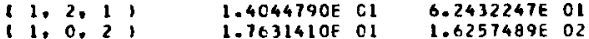

1. c. $-3,1, \cos 1-1 L_{1}+4 L_{2}-1 \omega_{1}+0 n_{2}$

100.10 CC

$c^{2} \mathrm{C}$

$0^{2}$

$0.7843831 E$ Ol $\quad-9.9051600 E$ OI

$-4.7564527 E 02$

$1043473 E$ 02 $1.0458558 E 03$

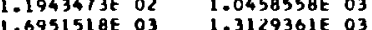

OC/N

SOE $02 \quad-7.3759512 E$ OS

$-3.1231448 E$ OS $-2.2415436 E$ O4

$4.6490638 E$ O3 $7.7880427 E$ O4

$1.2106260 E$ O4 $9.7768761 E$ O4

1 1. c, $-2,1, \cos 1+\mathrm{OL}_{1}+3 \mathrm{u}_{2}-1 \mathrm{w}$, ow

$11.0 .11 \quad-1.8509733 E$ CC $-9.6034817 E$ OC

$\mathrm{C}^{2} \mathrm{C} \quad \mathrm{C} / \mathrm{N} \quad \mathrm{DC} / \mathrm{N}$

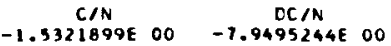

$C / N=N$

$-1.2683088 E 00$

1. $c,-1,1, \cos 1+1 \mathrm{~L}_{1}+2 \mathrm{~L}_{2}-1 \mathrm{H}_{1}+\mathrm{Om}_{2}$,

C c c c

2

$5 \mathrm{C}$

C/N

$C, N \circ N$

(1.0.1) -1.5688525E CC $-7.6761151 \mathrm{E}$

$-4.9710136 E$ ol

$-8.6899143 E-01$

$-4.2518200 E 00$

$8133660 E-01$

$\because 1,0 .-C_{0} 11 \cos 1+2 L_{1}+1 L_{2}-I_{1}+O w_{2}$,

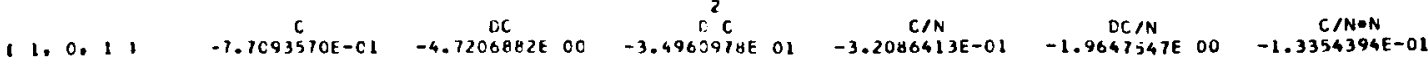

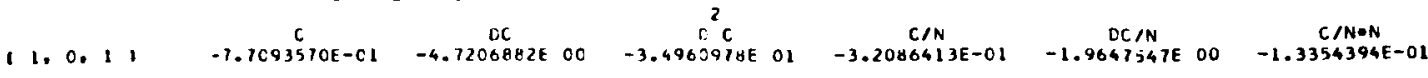

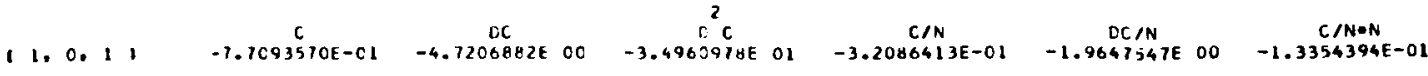

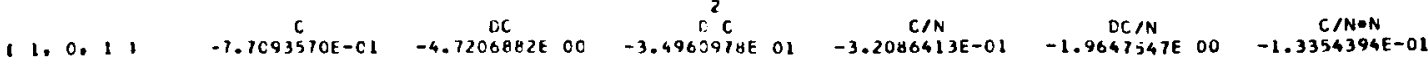




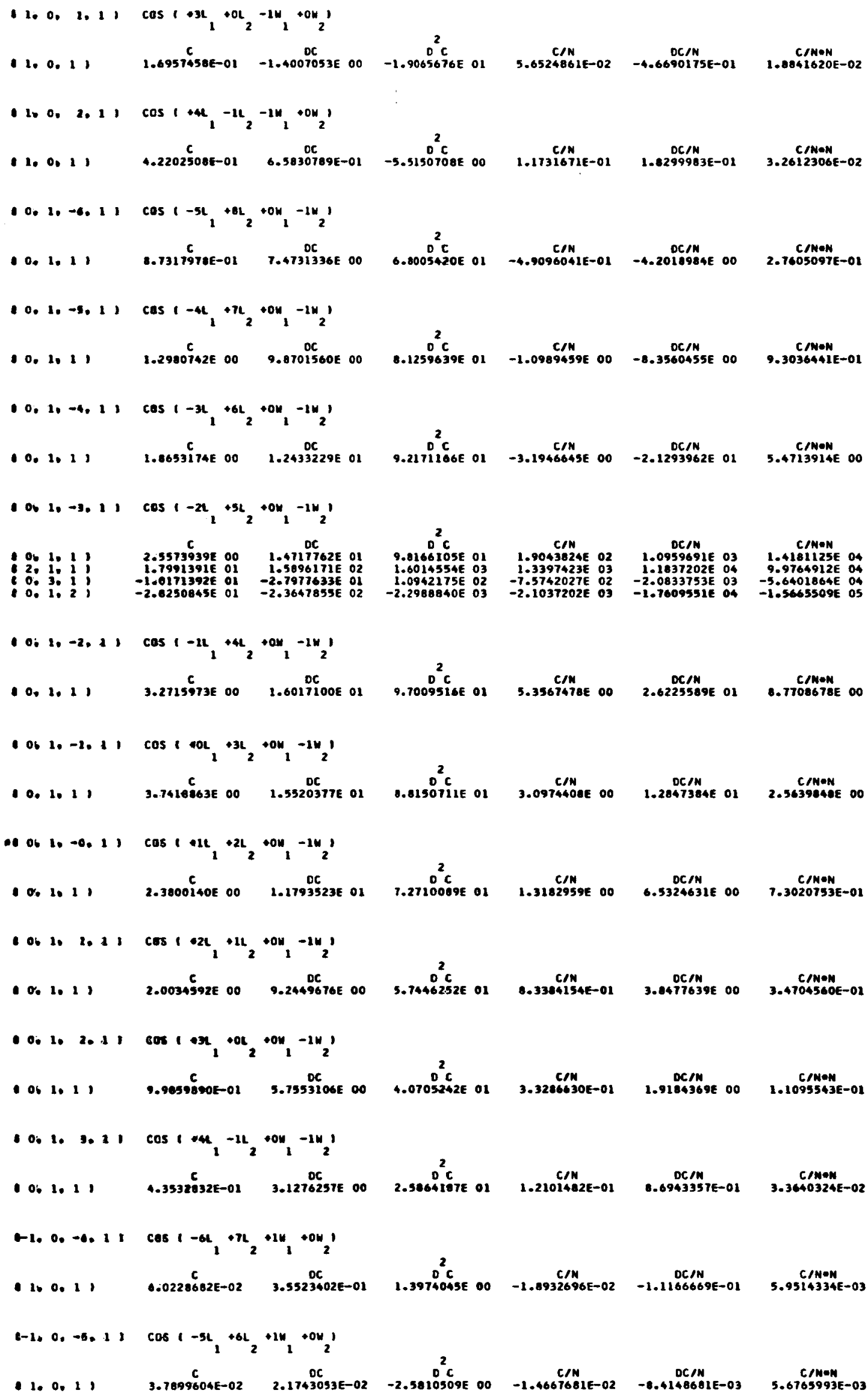


$0-1,0,-4,1) \cos 1-4 L_{1}+5 L_{2}+1 n_{1}+0 w_{2} 1$

1. 0.1 , $-4.6793540 E-02 \quad-7.3759640 E-$

1-1. $0 .-3,1, \cos \left(-u_{1}+4 L_{2}+1 y_{1}+0 w_{2}\right)$

10. $0.11 \quad-2.5040953 E-01-2.1017810000$

$110.11 \quad-2.5848953 E-01 \quad-2.1617810 E$ 0O

$-1.0,-2.11 \quad \cos 1-2 L_{1}+3 L_{2}+1 w_{1}+0 w_{2}{ }^{\prime}$

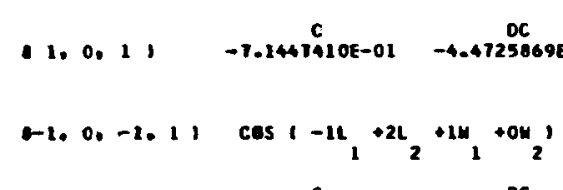

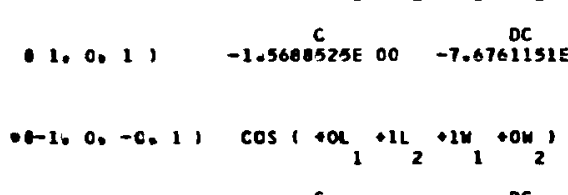

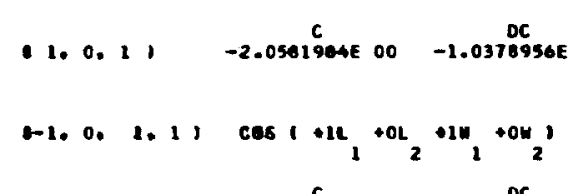

1. 0.1 , -3.3072795E 0O $-1.3951525 E$ ol

$1+160.2 .11 \cos 1 \cdot 22_{1}-1 L_{2} \cdot 1 x_{1}+0 w_{2}$

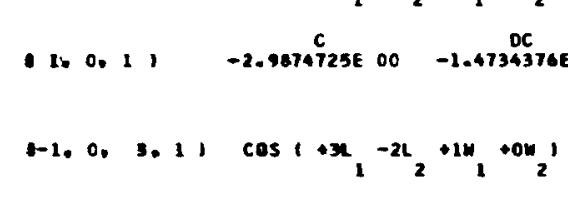

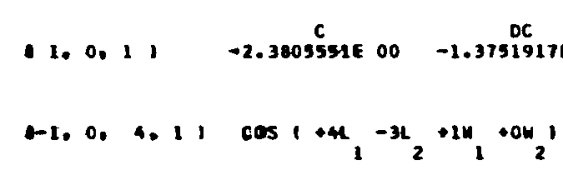

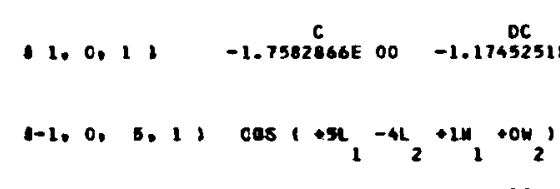

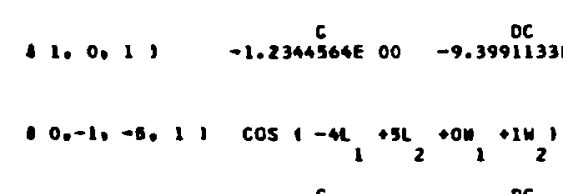

$\begin{array}{ll}10.1 .1) & -2.2075301 E-01 \\ 10 .-1 .-4.11 & \cos \left(-3 L_{1}+4 L_{2}+0 w_{1}+1 w_{2}\right)\end{array}$

10.1 .1 , $-2.7529090 E-C 1 \quad-1.3263389 C^{O C}$

$0,-2,-3,1, \cos \left(-2 t_{1}+3 L_{2}+0 w_{2}+1 w_{2}\right)$

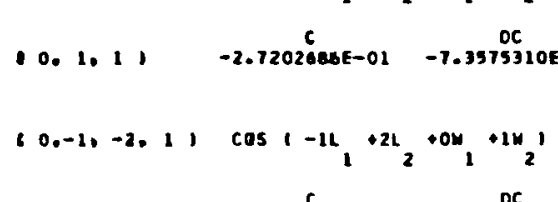

$10.1 .1,-1.3790020 E-01 \quad 6.2441500 E-01$

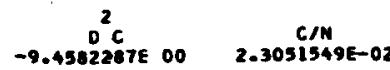

2.3051549E-02

3.112912

$-1.94390270^{2} \mathrm{C}$ O1 $\quad$ 1.8006317E-01

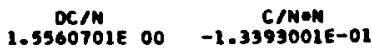

$\begin{gathered}0^{2} O \\ -3.3067208 E \text { OI } \\ C / N H\end{gathered} \quad 9.0217915 E-01$

OC/N
$5.6476150 E$
CANAN

$-4.9770136 E$

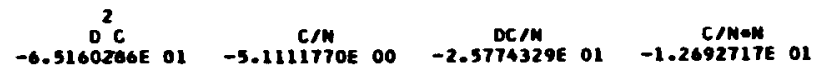

$0^{2} \mathrm{C}$

$0_{0}^{2} c$

C/N

C/N

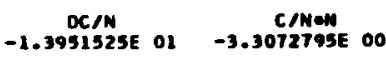

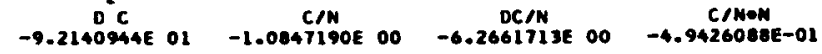

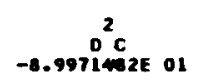

$-1-070309$

C/M 00

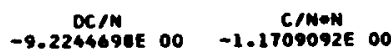

$\mathrm{O}^{2} \mathrm{C}$
$-0.7305098 \mathrm{C}$ OI
$\mathrm{CHN}$

$0^{2} \mathrm{C}$
$-0.7305098 \mathrm{C}$ ol
$\mathrm{C}$

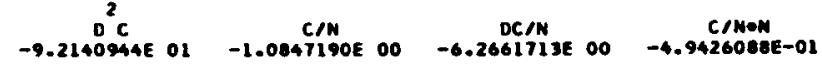

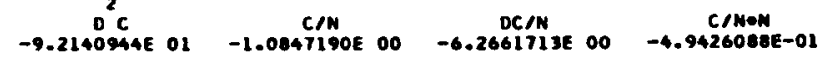

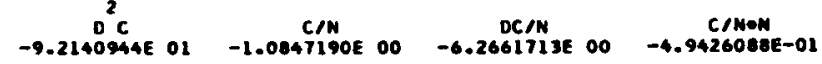

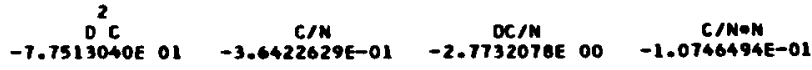
$-0$
$0^{2} \mathrm{c}$

${ }^{2} c$

$\underset{1.1514968 E-01}{C / M}$

DC/M

crmon

$2 \mathrm{c}$

C/M
$1.9016271 E-0$

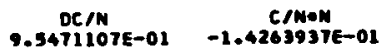

1.7635016

$0^{2} \mathrm{C}$
$1.7635616 \cos ^{2}$

C/N

$3.4349315 E-01$

9.2904851E-01

CINAM

$373491 E-01$

$0^{2} \mathrm{C}$

$1.2353105 E$ OI

$C / N$

$\underset{-3.2002461 E}{\text { OC/M }} 00 \quad \begin{gathered}\text { C/MON } \\ -3.6404303 E\end{gathered}$ 
$0,-1,-1,1, \cos 140 L_{1}+1 L_{2}+0 w_{1}+1 w_{2}$

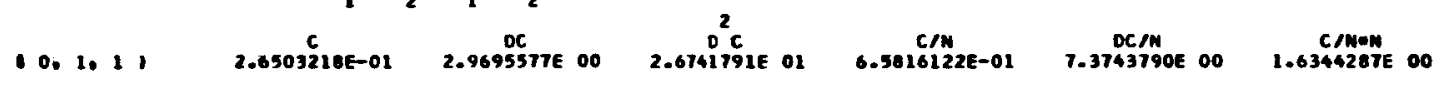

$0 .-1,-0,1, \cos 1+1 L_{1}+0 L_{2} * 0 w_{1}+1 w_{2}^{\prime}$

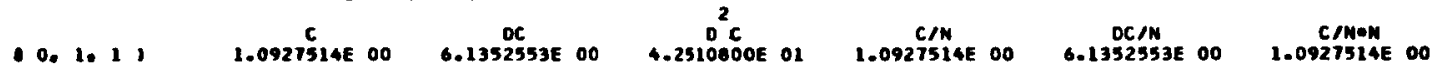

$0 ;-1,1,1, \cos \left(+2 L_{1}-12_{2}+0 n_{1}+1 n_{2}\right)$

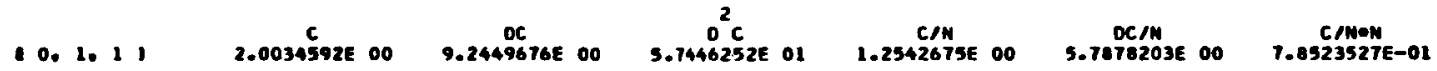

$0,-1,2,1, \cos \left(+3 L_{1}-2 L_{2}+0{ }_{1}+1 w_{2}\right.$

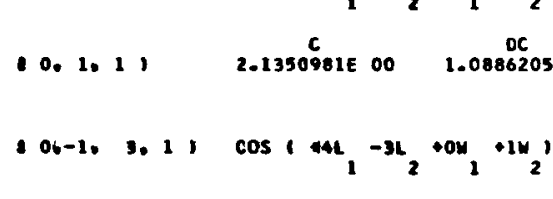

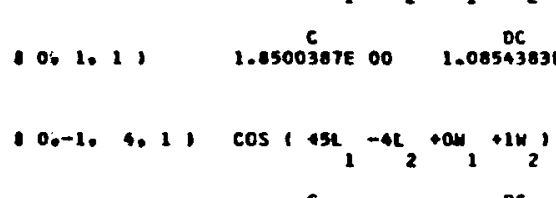

$0.1 .1, \frac{c}{c}, 1.4371941500 \quad 0.0813150$

Ob 1.1, $1.0430030 E$ 00 $7.9859648 E$ OC

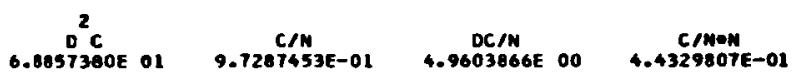

$0:-10,1, \cos \left(+\pi \mathrm{L}_{1}-6 \mathrm{~L}_{2}+0 \mathrm{w}_{1}+1 \mathrm{w}_{2} 1\right.$

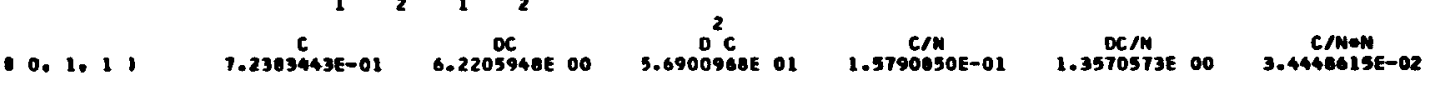

$1460 .-12.01 \cos \left(-4 t_{1}+12 L_{2}-4 x_{1}+0 w_{2}{ }^{\prime}\right.$

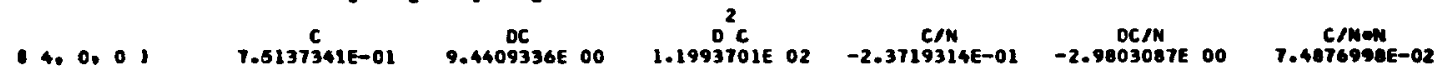

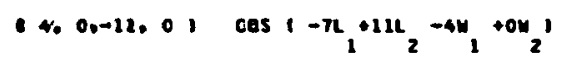

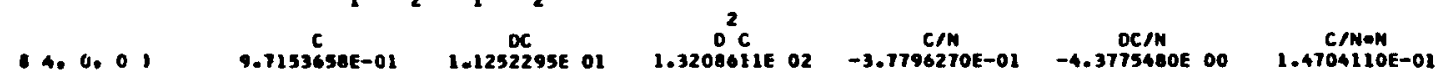

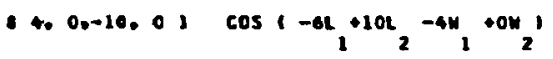

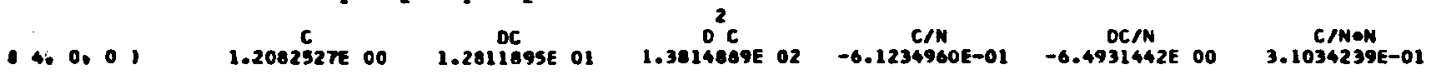

14 $0,-0.02 \cos \left(-5 t_{1}+9 t_{2}-4 x_{1}+0 w_{2}^{\prime}\right.$

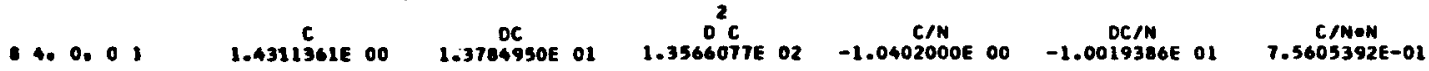

1. 4. $0 .-4.01 \cos \left(-4,+4 t_{2}-4 t_{1}+0 n_{2}\right.$

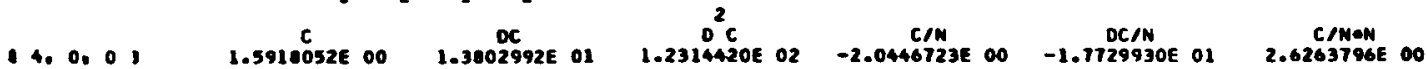

4. $0 .-7,0, \cos \left(-3 L_{1}+7 L_{2}-4 H_{1}+0 N_{2}^{\prime}\right.$

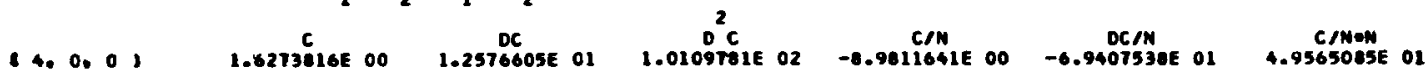


$140,+6,01 \cos \left(-2 \alpha_{1}+6 L_{2}-4 w_{1}+0 w_{2}\right)$

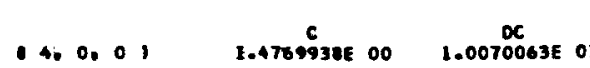

$440,-5,0, \cos 1-11,-5 L_{2}-411+0 w_{2}$

4. $0.0,1.1179434500 \quad 6.6950263 E$

$$
0^{2} \mathrm{c}
$$

0
$0.37907 a b E$
C/N

$1.1031295 E$ O0
OC/N

$.5964420 E$ OO
CrNow

1.00asilge 00

4. 4. $0.2 .0, \cos 14 \mathrm{~L}_{1}-\mathrm{uL}_{2}-4 \mathrm{u}_{1}+0 \mathrm{w}_{2}$

4. $0.0, \quad-1.33039508-02 \quad-3.4738005 E-02$

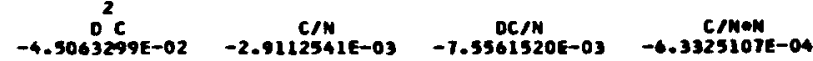

$361+11,0, \cos \left(-8 L_{1}+12 L_{2}-3 W_{1}-1 W_{2}\right)$

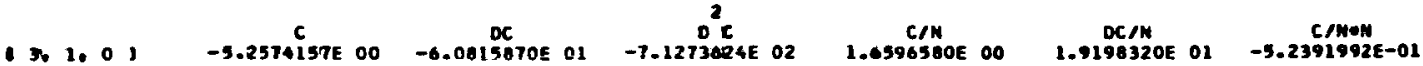

1 30. 10-10, 0 , cas $1-n_{1}+11 L_{2}-3 w_{1}-1 H_{2} 1$

$1,0,0$ C DC

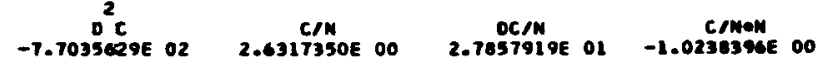

$1361 . \rightarrow 0,0, \cos 1-6 e_{1}+10 L_{2}-3 w_{1}-1 w_{2}$

1. 1. 0, c 0 DC

- $3.1 .-x, 0$, $\cos 1-x_{1}+x_{2}-3 x_{1}-1 x_{2}$

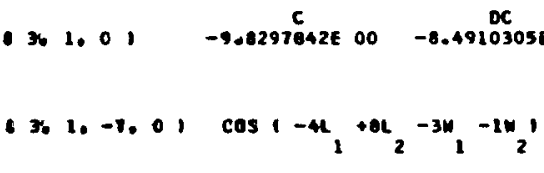

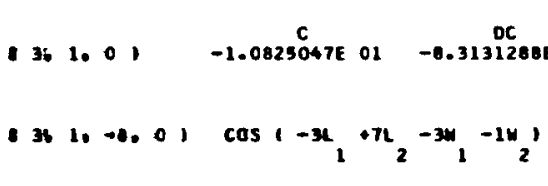

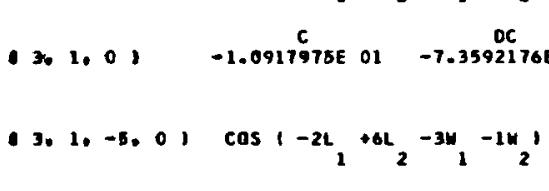

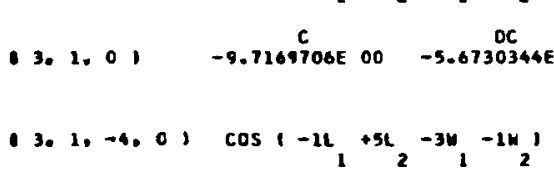

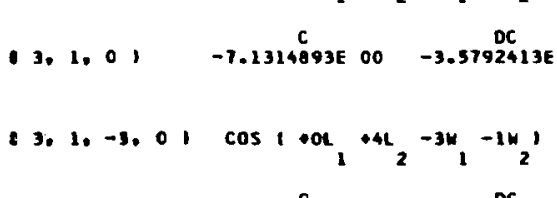

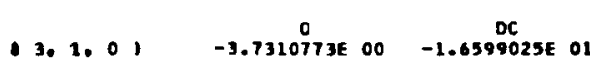

$0^{2} \mathrm{c}$

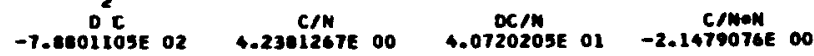

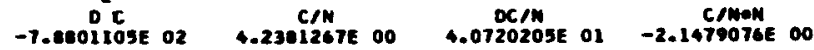

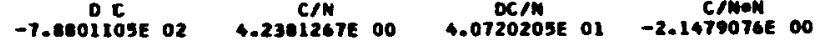

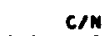

DC/N

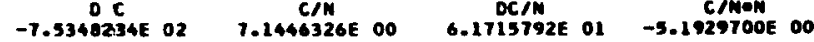

1. $1,1,0, \cos \left(44 L_{1}, 0 L_{2}-3 H_{1}-1 \psi_{2}\right.$

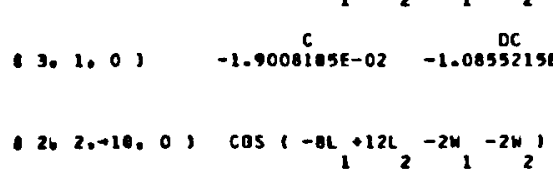

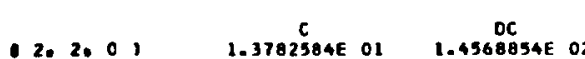

$0^{2} \mathrm{C}$

$0^{2} \mathrm{C}$

C/N
$1.3904762 E$ 01

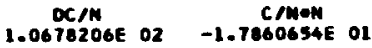

T)



12. 2. -9. $01 \cos \left(-\pi \pi_{1}+21 L_{2}-2 w_{1}-2 w_{2}\right)$

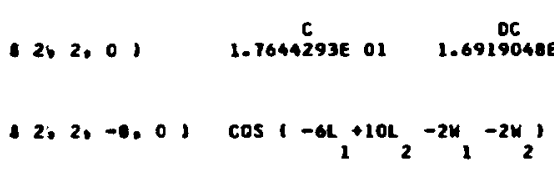

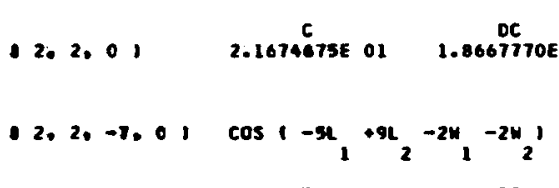

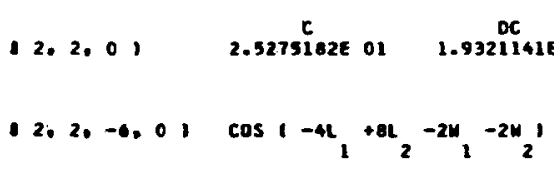

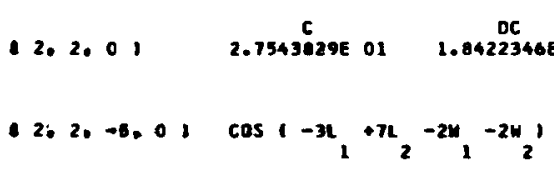

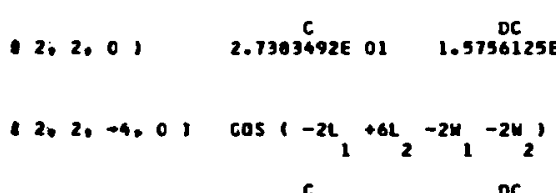

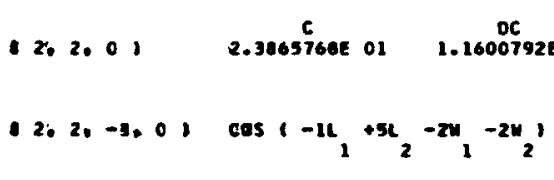

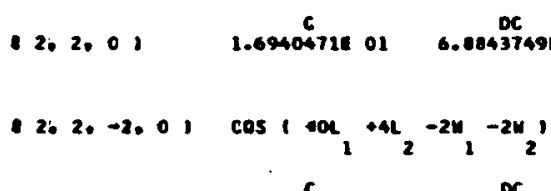

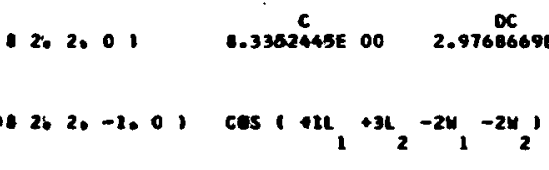

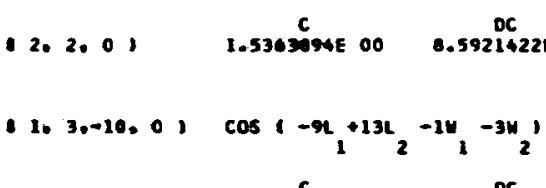

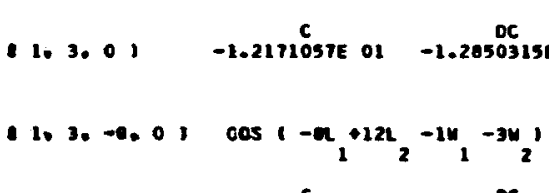

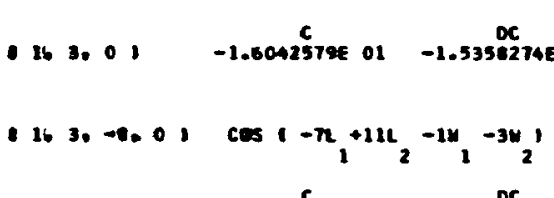

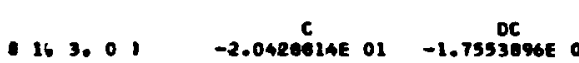

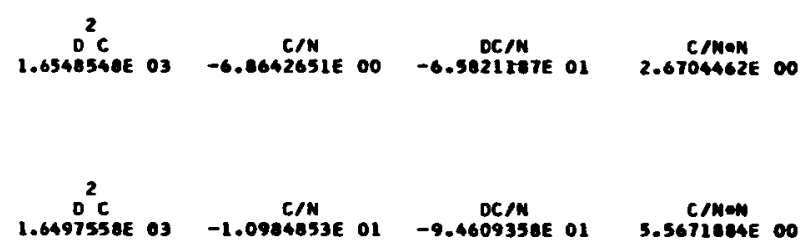

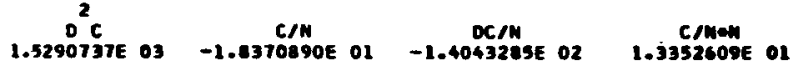

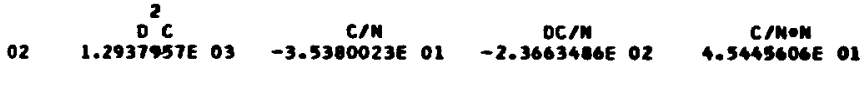




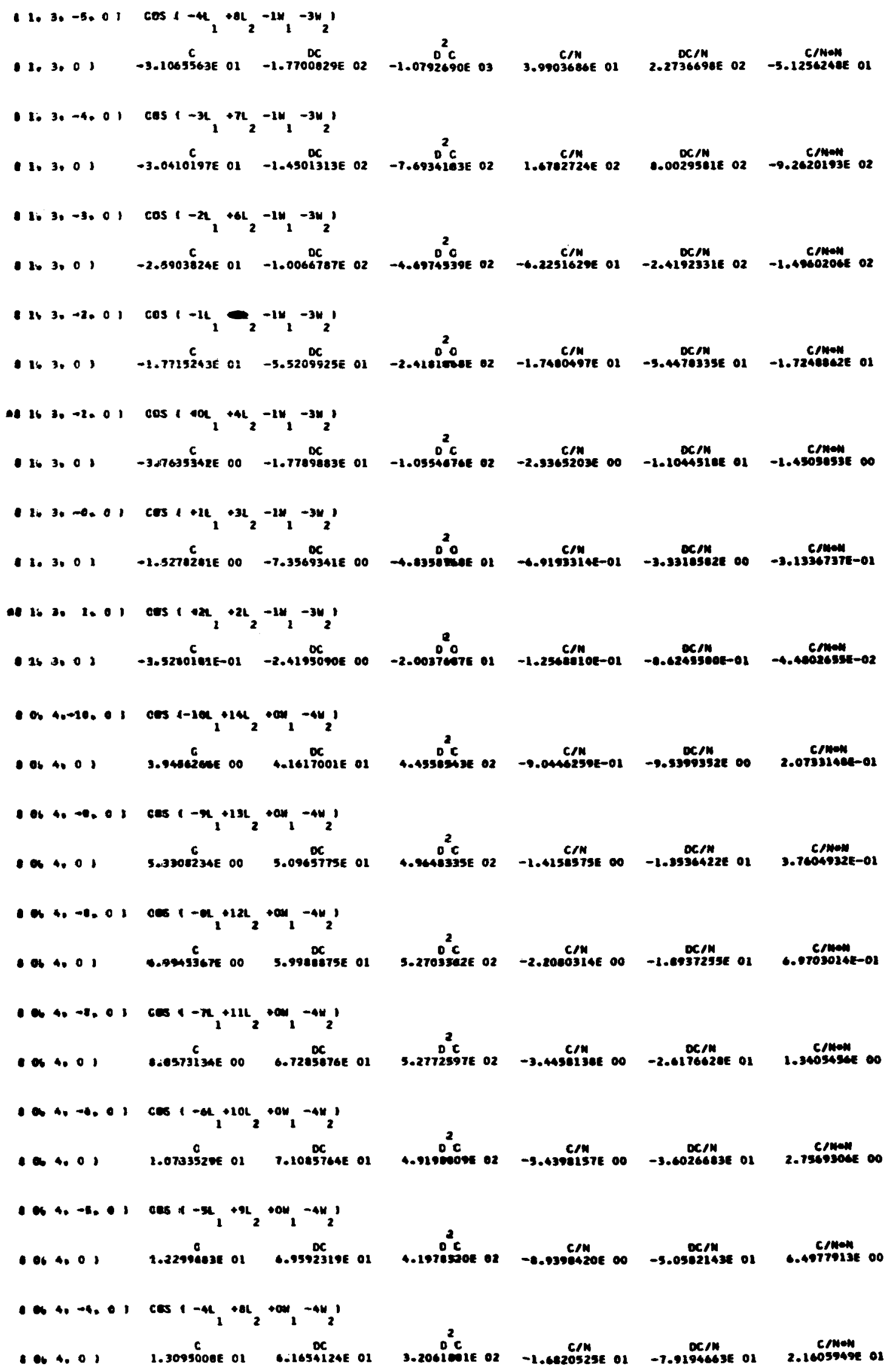




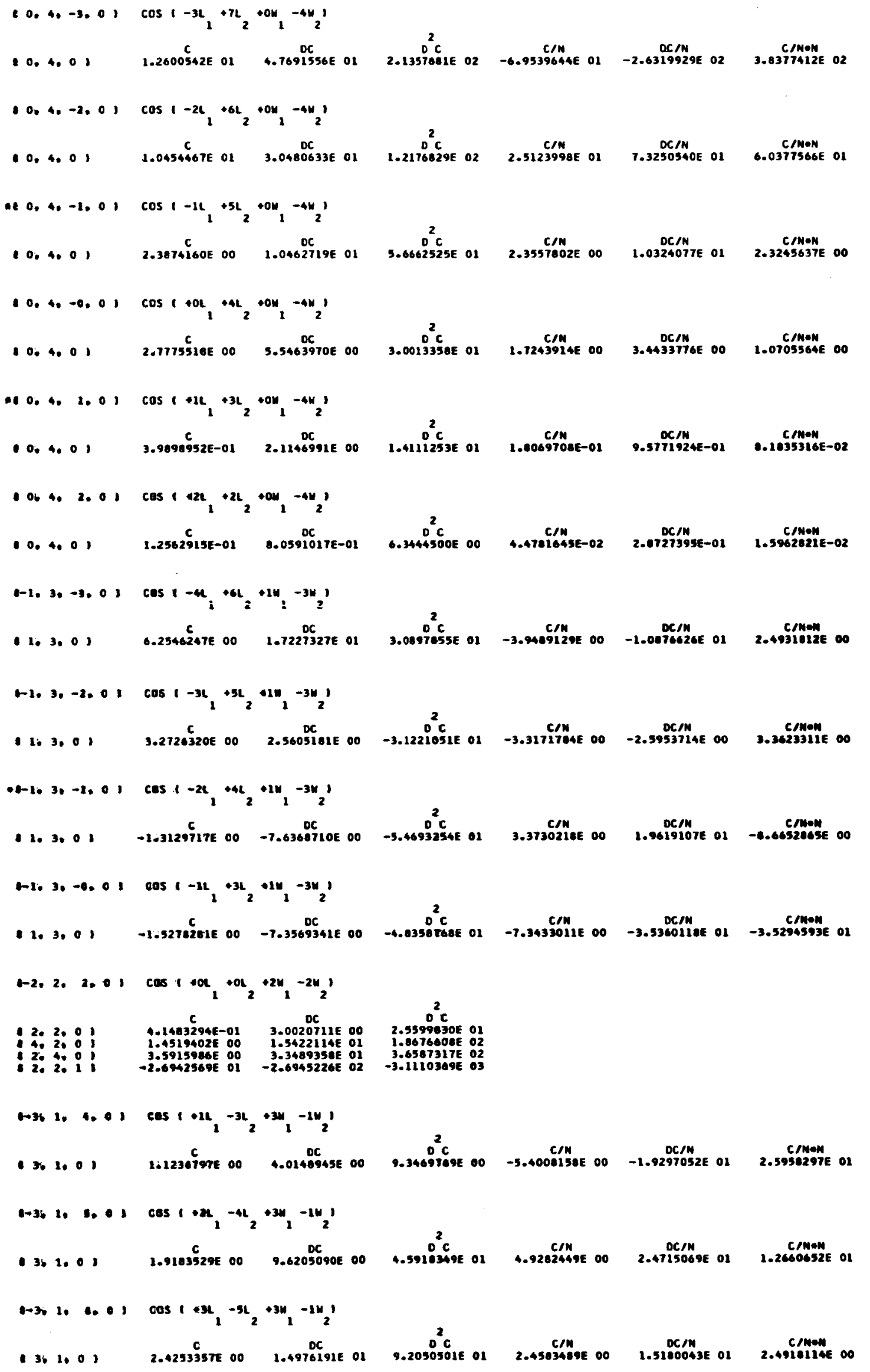


12. $\left.0,-7,1, \cos 1-\mu_{1}+0 L_{2}-2 w_{1}+0 w_{2}\right)$

10.0 .1100

12. 0.11 2.1852112E 00 2.1421179E 01

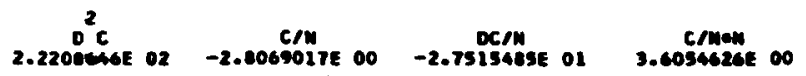

12. $0 .-4.11 \cos \left(-x_{1}+n_{2}-2 w_{1}+0 w_{2}\right)$

$1260.11 \quad 2.1249671600 \quad 2.42606070^{6}$

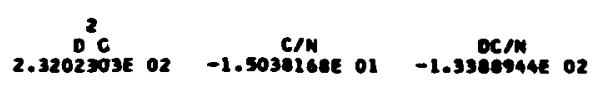

CAMeN

$-260 .-5.11 \cos \left(-x_{1}+\mathrm{ci}_{2}-2 w_{1}+0 w_{2}\right.$

20,100

$0260.113 .167334800 \quad 2.54963016$ ol

$0^{2} \mathrm{c}$

ocm

0.127246e or 1.0292273E or

$1.1,-4,11 \cos 1-\mu_{1}+\mu_{2}-2 \omega_{1}-2 \omega_{2} \mid$

- 10.0100

0161.11 -8.102465se oo -7.136637te or

$-4.730^{2} \mathrm{c} c$

$c / m$

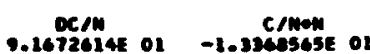

$\left.1010-5,11 \cos 1-x_{1}+72_{2}-1 x_{1}-1 x_{2}\right)$

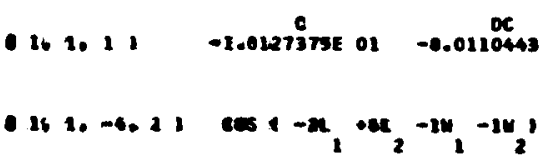

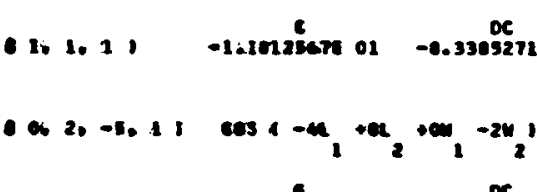

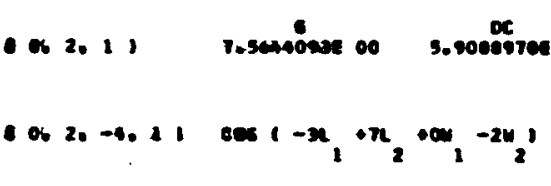

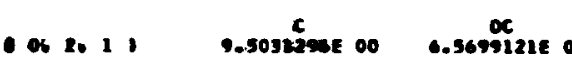

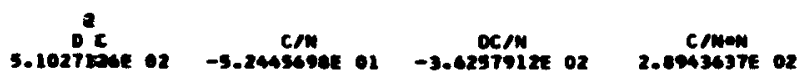

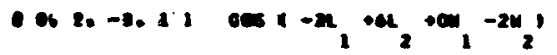

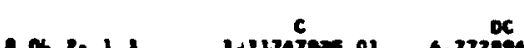

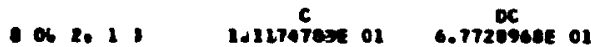

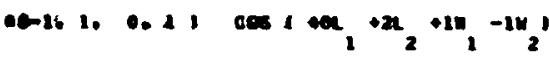

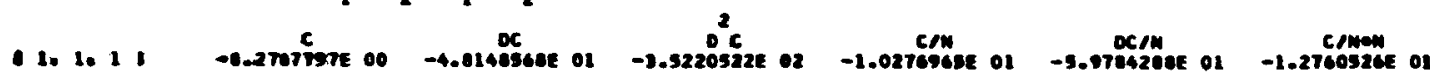

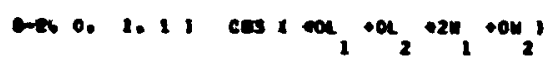

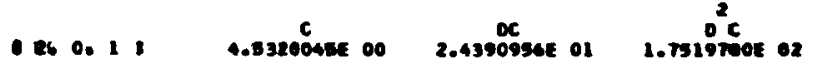

$-1,-1.0 .11$ ons $1400_{1}+0 e_{2}+1 w_{1}+1 w_{2}$

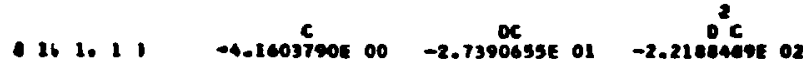

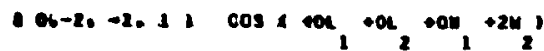

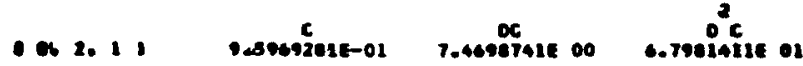

- st $0 .-0.01 \cos 1-44_{1}+9 L_{2}-5 w_{1}+0 w_{2}$

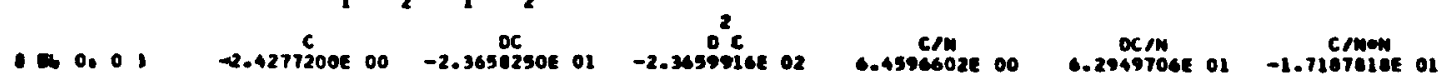


5. $0,-6.01 \cos 1-3 L_{1}+\sigma_{2}-5 H_{1}+0 w_{2}$

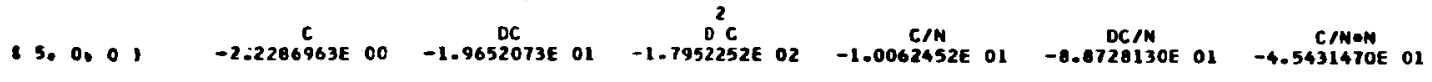

4. 4. 1, -9. $0, \cos 1-5 t_{1} 10 \varepsilon_{2}-4 x_{1}-1 w_{2}^{\prime}$

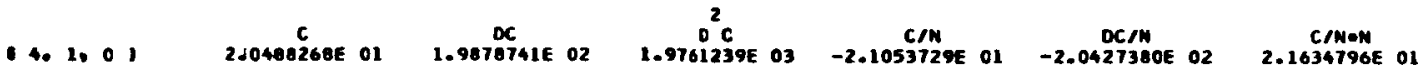

16. 1. $\left.-0.01 \cos 1-4 L_{1}+9 L_{2}-4 x_{1}-1 n_{2}\right)$

C
$2.0431840 E$ or
$1.0,0 C$
$0.7809733 E$

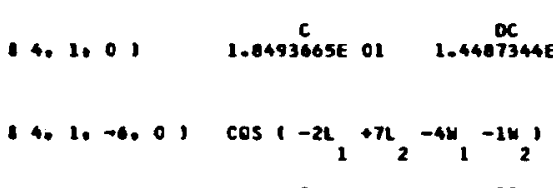

4. $1.01 C$

$362,-4.0, \operatorname{ces} 1-5 t_{1}+10 L_{2}-3 w_{1}-2 w_{2}$

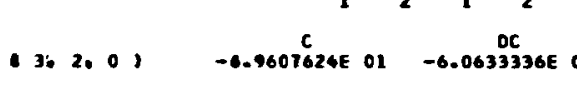

1.010

$0^{2} \mathrm{C}$

03

C/N DC/N

C/NON

1.2. $2,-7.0, \cos 1-4,+9 L=-3 w_{1}-2 w_{2} 1$

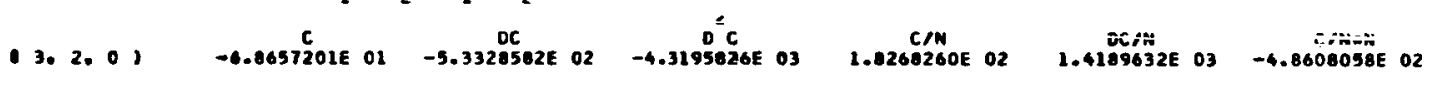

3. $2,-6,0, \cos 1-3 u_{1}+\alpha_{2}-3 x_{1}-2 u_{2}$

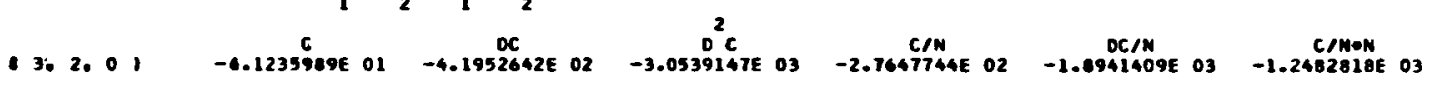

13. 2, $-5,0, \cos 1-2 \mathrm{~L}+7 \mathrm{~L}_{2}-3 \mathrm{H}_{1}-2 \mathrm{H}_{2}$

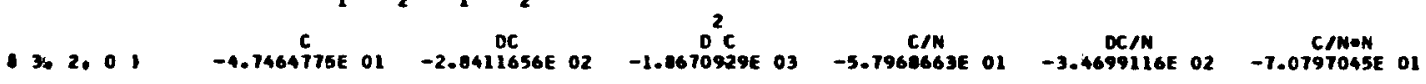

1 2. $3,+1,0, \cos \left(-5 t_{1} 10 L_{2}-2 w_{1}-3 w_{2}\right.$

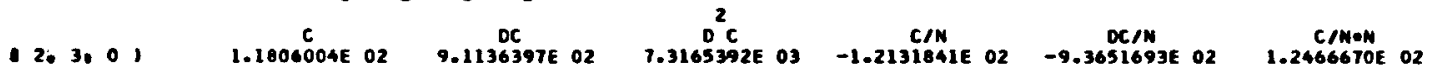

2. 3. $-6,0, \cos 1-4 L_{1}+9 L_{2}-2 x_{1}-3 w_{2}$

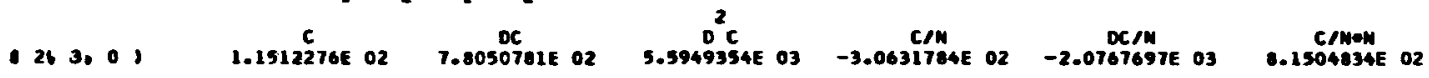

- 26. $30-5,0, \cos \left(-3 t_{1}+8 t_{2}-2 w_{1}-3 x_{2}\right)$

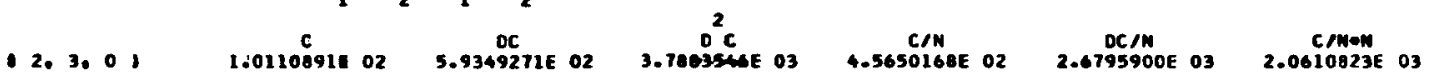

1 2. 3. $-4,0, \cos \left(-2 u_{1}+7 L_{2}-2 w_{1}-3 w_{2}\right)$

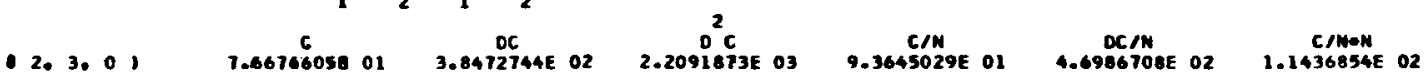

- It 4. $-4.01 \cos \left(-5 t_{1}+10 \mathrm{~L}_{2}-1 w_{1}-4 w_{2}\right)$

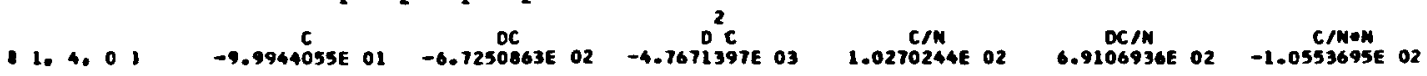


1. 4. $-5,0, \cos -4 \alpha_{1}+9 L_{2}-1 \mu_{1}-4 n_{2}$

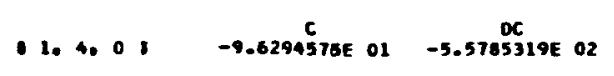

1. 4. $-4.01 \cos 1-3 x_{1}+6 L_{2}-1 w_{1}-4 \omega_{2}^{\prime}$

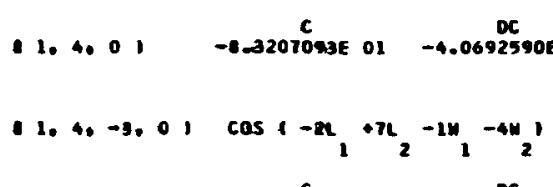

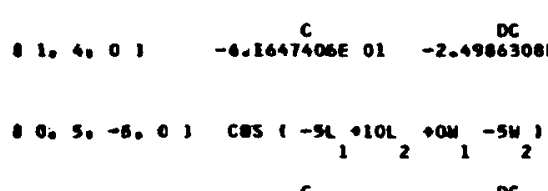

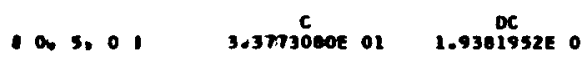

- $065 .-4.01$ os $1-4 L_{1}+x_{2}+0 y_{1}-5 x_{2}$

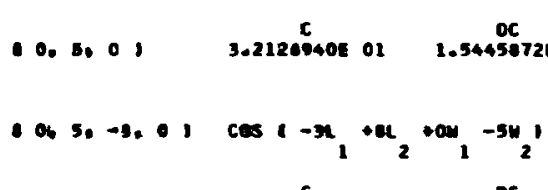

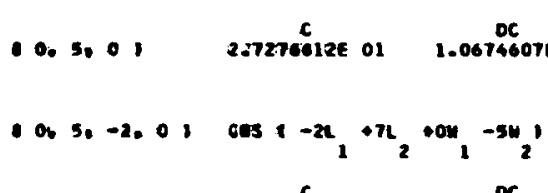

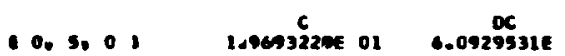

$-8-16$ if $-2,0,000,-n_{1}+5 x_{2}+1 w_{1}-4 w_{2}$

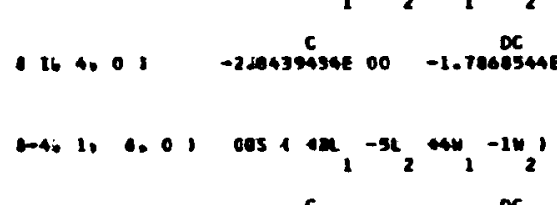

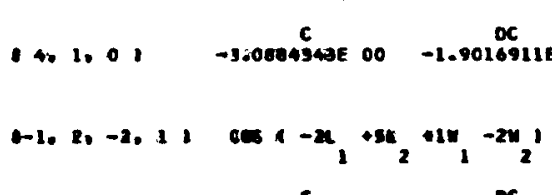

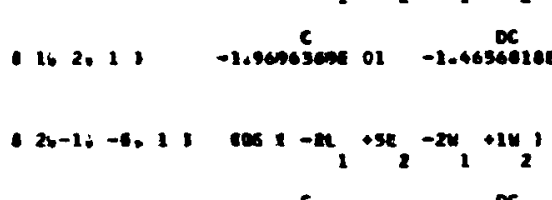

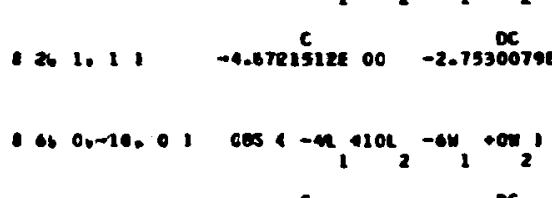

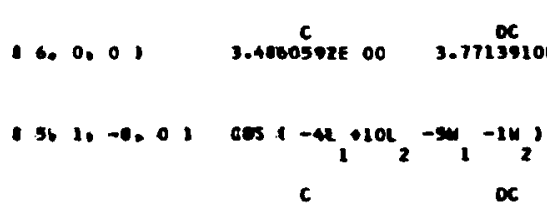

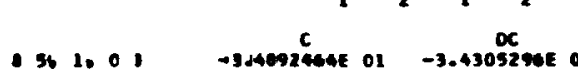

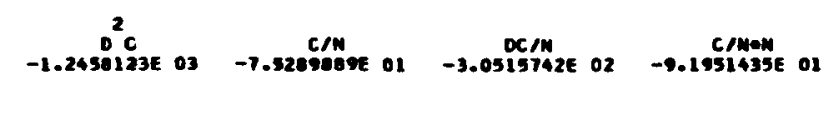

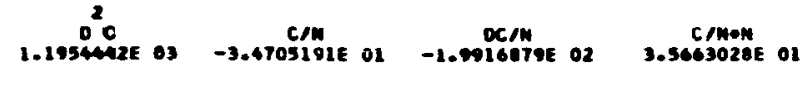

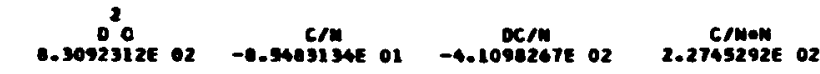

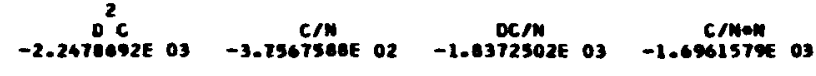

907157

$\underset{2.3621993 E}{C / m} 02$

DC/N
$.4043319 E$ O3

CINen

-6.0174819 OE

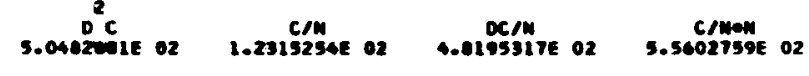

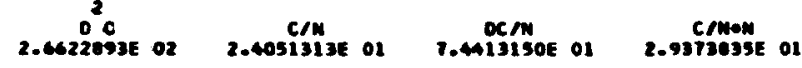


13. 3. $\left.-7,01 \cos 1-4 L_{1}+10 L_{2}-3 H_{1}-3 W_{2}\right)$

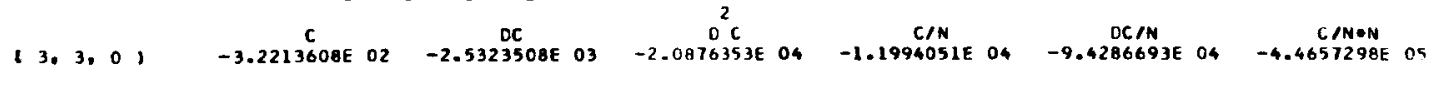

$\left.12.4,-6.01 \cos 1-4 L_{1}+10 L_{2}-2 W_{1}-4 W_{2}\right)$

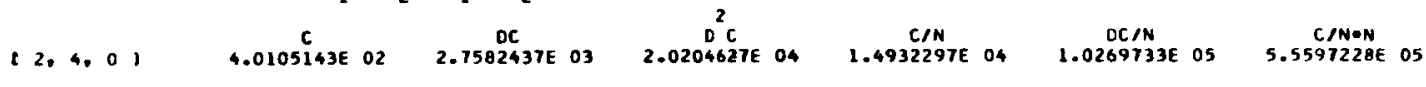

1 1.5.-5. $c_{1} \cos \left(-4 L_{1}+10 L_{2}-1 W_{1}-5 W_{2}^{\prime}\right.$

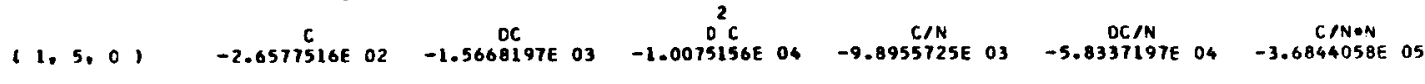

$10.6,-4,0) \cos \left(-4 L_{1}+10 L_{2}+0 w_{1}-6 w_{2}\right)$

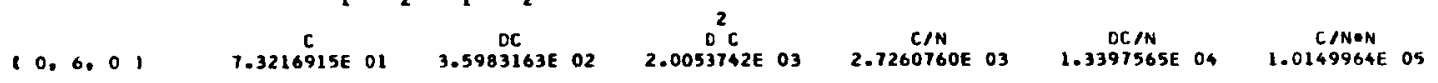

1-3. 3, 3, O, $\left.\cos 1+\mathrm{OL},+\mathrm{OL}_{2}+3 \mathrm{H}_{1}-3 \mathrm{w}_{2}\right)$

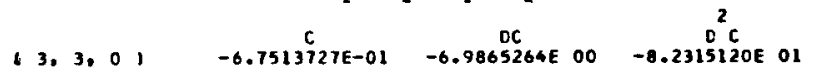

1. 4. $0 .-9,1, \cos 1-4 L_{1}+10 L_{2}-4 H_{1}+0 W_{2}^{\prime}$

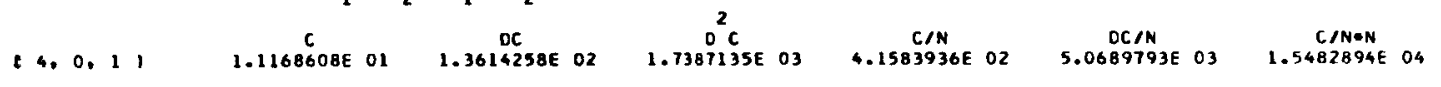

13. $\left.1,-8,1, \cos 1-4 t, 10 L_{2}-3 \omega_{1},-1 w_{2}\right)$

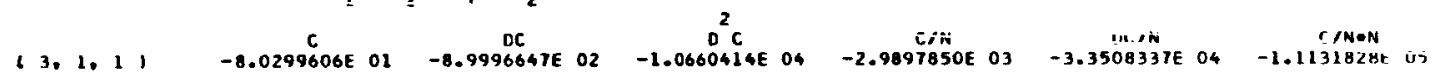

(2. 2. $-7,1, \cos \left(-4 t_{1}+10 L_{2}-2 w_{1}-2 w_{2}\right)$

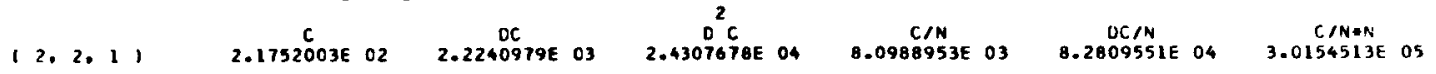

(1. 3. $-6,1) \cos \left(-4 L_{1}+10 L_{2}-1 H_{1}-3 H_{2}\right)$

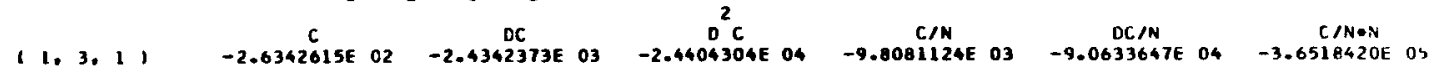

$10,4,-5,1, \cos 1-41_{1}+10 L_{2} \cdot 0 x_{1}-4 x_{2}$

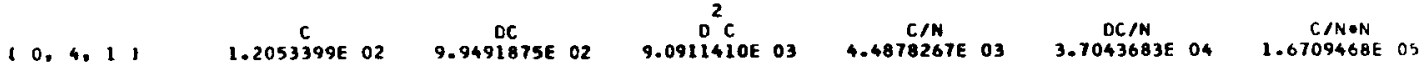

1-3, $1,2,1, \cos 1+\mathrm{OL}, \mathrm{CL}_{2}+3 \mathrm{H}_{1}-1 \mathrm{H}_{2} 1$

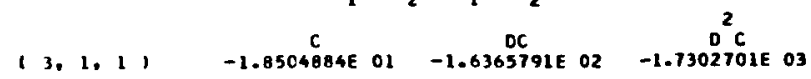

$\left(1,-3,-2,11 \cos 1+0 L_{1}+0 L_{2}-1 w_{1}+3 W_{2}\right)$

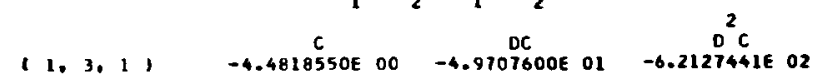

$11,1,-7,2) \cos \left(-4 L_{1}+10 L_{2}-1 H_{1}-1 H_{2}\right)$

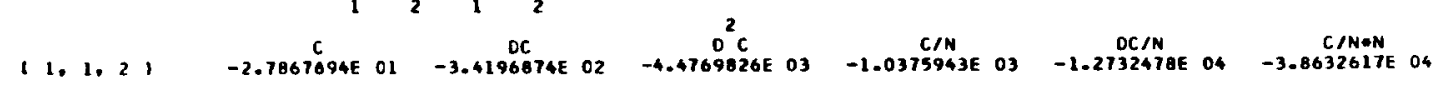


Appendix II 
CONYENTIONAL RELATIVE COOROINATES, INMER PLANET

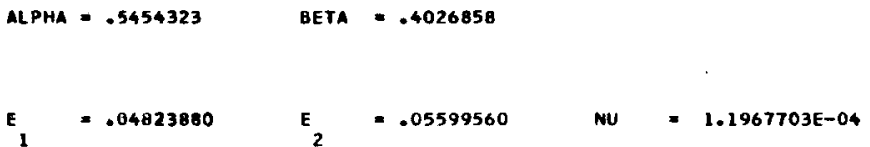

C DC

${ }^{2}$

$x \cdot \times \times \times \times \times \times \times E \rightarrow-$

$x \cdot \times \times \times \times \times \times \times E--$
DC/N

CrMan

$\mathrm{CS}_{1} \cdot \mathrm{s}_{2}, \mathrm{~s}$

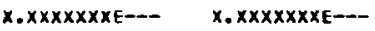

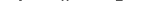

$x-\times \times \times \times \times \times \times E=$

$x \cdot \times \times \times \times \times \times \times E=$

$\left.\cos 1+\mathrm{OL}_{1}+\mathrm{OL}_{2}+\mathrm{OH}_{1}+\mathrm{OH}_{2}\right)^{\prime}$

C

1.0901656E 00

5. $0566100 \mathrm{E}-04$

$6,0135 \leq 315-0$

$6.4957340 \mathrm{E}-07$

$8,4317204 \mathrm{E}-0$

$5.5694967 \mathrm{E}-0$

$3.1903996 \mathrm{E}-0$

$\rightarrow, 0032291 \mathrm{E}-0$

$1,4269038 \mathrm{E}-07$

5. $1950744 \mathrm{E}-08$

$-1.0402490 \mathrm{E}-04$

$-1.2872983 \mathrm{E}-06$

$-1.7345787 \mathrm{E}-06$

$-6.1118609 \mathrm{E}-0$

$-5.6004616 \mathrm{E}-0$

$2.0605719 E-09$

2.776531 AE $=0$

$-4.2314652 \mathrm{E}-11$

DC

2. $2065981 E-01$

$1.8253456 E-03$

$2.4595741 E-03$

$5.0560257 E-06$

$5.2392908 E-0$

$2.9974673 \mathrm{E}-0 \mathrm{~S}$

$1.4475753 \mathrm{E}-0$

$4.1654135 E-07$

$1.2538550 \mathrm{E}-06$

3. $8995840 \mathrm{E}-07$

$-3.7551122 \mathrm{E}-04$

$-7.9989966 \mathrm{E}-06$

$-1.0778301 E-05$

$-6.3594730 \mathrm{E}-0 \mathrm{~B}$

-5.2916167E-07

$-2.5794383 E-07$

$.8891960 \mathrm{E}-07$

(9)

$-4.1969405 \mathrm{E}-10$

$0,0.3$

$\cos (+04+01+14,-14$,

$1-1,1,1,01$

$\cos 1+0$

$1^{+0 L}$

$+1 w_{1}-1 H_{2}$

$\left\{\begin{array}{lll}1,10 & 0 \\ 3 & 1 & 0\end{array}\right\}$

( $1,3,0$

(5. 1.0)

3, 3, 01

1.1:1

(1; $3,1,2$,

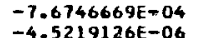

$-4.5219126 \mathrm{E}-06$

$-1.3659013 E-0$

$-1.4840086 E-0$

$-1.6469622 \mathrm{E}-0$

$2.5979774 E=0$

$3.6035192 \mathrm{E}-0 \mathrm{~B}$

$7.7705589 E-0$

$-4.5110169 E=09$

$$
\text { DC }
$$

$-3.4648426 E-03$ $-3.1981862 E-05$ $-7.5614528 \mathrm{E}-05$ $-1.5444434 E-07$ $-1.4321933 \mathrm{E}-06$ $-1.3740843 E-06$ $1.1294322 \mathrm{E}-0$ $3.6033505 E-O T$ $7.2042917 E-07$ $-4.3972330 E-08$

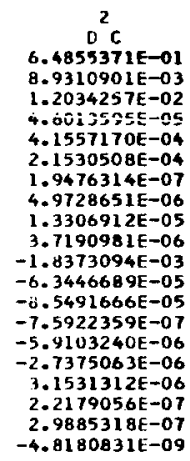

$t-2 ; 2,2,0, \cos 1+0 \mathrm{~L}+\rho \mathrm{L}+2 \mathrm{w}-2 \mathrm{w}$,

(2, 2, 0 )

(4:2,0)

i 2. 2: 1

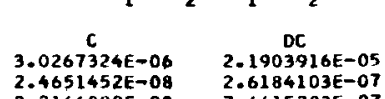

$2.1903916 E-05$

$2.6615283 E-07$
-.6615285

$-2.3528457 E-07$
$0^{2} \mathrm{C}$

$-1.9010860 E-02$ $-2.7364370 E-04$ $-5.8580624 E-04$ $-1.9628577 \mathrm{E}-06$ $-1.618 B 544 E-05$ $-1.3947969 E-05$ $1.4118078 E-04$ $4.1820418 \mathrm{E}-06$ $7.9092686 \mathrm{E}-06$ $-5.0013851 E-07$

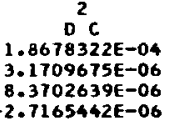




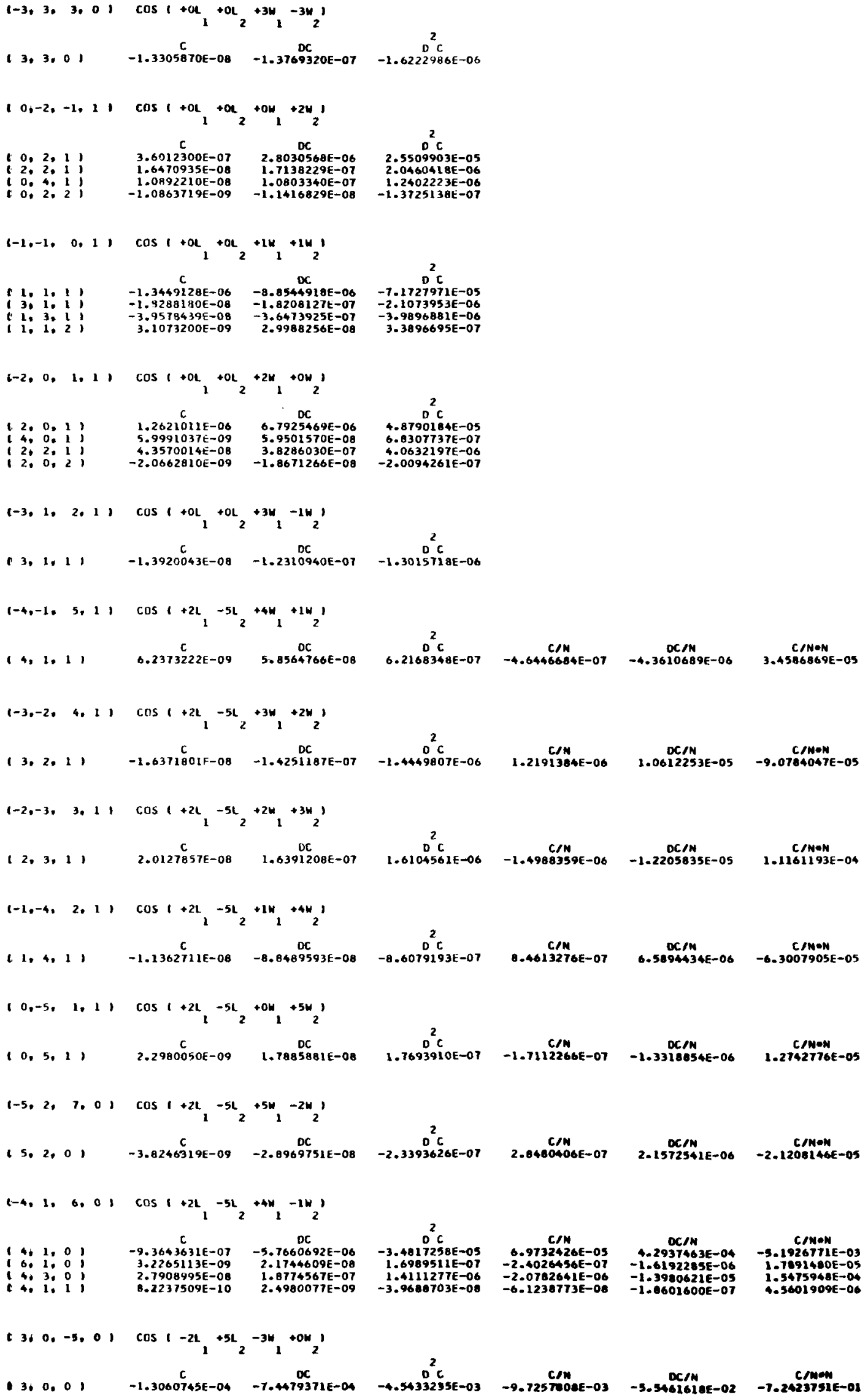




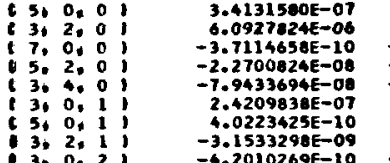

$1.4596744 E-06$ $-2.5306648 E-09$ $-1.3844705 E-07$
$--1.7574865 E-07$ $-4.7574865 E-07$
$1.9732274 E-06$ $1.9752274 E-06$ . $9.0820625 E-09$
$-4-7274230 E-09$
$3.71222295-06$ $-3.45766545=06$ $1.8211874 E-05$ 5.04060 5.0466760E-07 $2 .+67012058-04$
$-2.6852163 E-00$ $-1.0916931 \mathrm{E}-0$
2.5416335E-0S 4.3370350 -04 $-1.6904337 \mathrm{TE}-06$ -569150s92e-06 $1.6024036 \mathrm{E}=0 \mathrm{~S}$ 2. -2.3401 sose-0
1.000957 4t-04 2.2972210E-03 -3.5426970E-05 $1.4704670=04$ . $6.7830255 E-07$ $-1.0309562 E-05$

1.09264are-03 3.370537se -02 $-2.03006235-04$ -4.4047152E-a 1.3424711E-03 2.230445 $-1.7405674 E-0$

$12+1,-4,0) \cos \left(-2 L_{2}+5 L_{2}-2 w_{1}-2 w_{2}\right.$

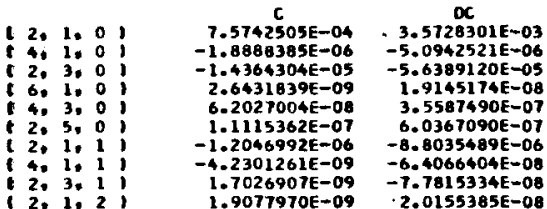

${ }^{2}$

$1.8612223 E-02$ $1.0219017 E-0$ $-1.8766712 \mathrm{E}-04$ $3.0577055 \mathrm{E}-06$ 4.5131310E-06 $-7.5195543 \mathrm{E}-05$ $-9.273543 \mathrm{E}-0 \mathrm{G}$ $-1.66607 B E-06$ 2.4225413E-07

2.0155385E-08
$1162,-3,0, \cos \left(-2 L_{1}+5 L_{2}-1 w_{1}-2 w_{2}\right)$

$(1,2.0) \quad-1.4543303 E-03 \quad-5.4296368 E=03$

3. 2. 0 , $\quad 3.3512307 \mathrm{E}-06 \quad 1.3524385 \mathrm{E}-06$

11,400

(1, 6, $0, \quad-7.8162754 E-00$

c $1,2,11$ 1.9243459E-06

1, 2, $1,1.4165770 \mathrm{E}-08$

$-6.5206986 E-08$

$-4.8744090 \mathrm{E}-07$

$-4.0092663 \mathrm{E}-07$

$1.2528208 E-05$

$1.7676885 E-07$

4.8919072E-09 1.3317660E-07

1, $2,2,1$

$1.3317660 E-07$
$-2.7634264 E-06$ $\left(0,3,-2,0, \cos 1-2 L_{1}+5 L_{2}+0 H_{1}-3 H_{2}\right)$

C. $0.0190 \quad$ C $\quad 9.2123446 E-04 \quad 2.5357557 E-03$

2.3, $3, \quad-1.7220229 \mathrm{E}-06 \quad 9.6631705 \mathrm{E}-06$

4. $3.01 \quad-5.0714760 E-06 \quad-6.9165147 E-06$

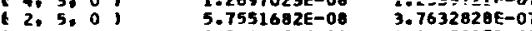

$0,7,0$

2, 3, 1

i. $0,3.2$;

$2.2997076 E-08$

$-9.6875168 E-07$

$-1.8919066 E-08$-5.6295741E-OB

$\begin{array}{ll}-4.5582971 E-09 & -2.0215961 E-07 \\ -6.7485511 E-00\end{array}$

$D^{2} \mathrm{C}$
$-2.3738627 E-02$
$-8.9558017 \mathrm{E}-05$
$4.9524172 \mathrm{E}-05$
$-8.6956331 \mathrm{E}-07$
$-4.7980460 \mathrm{E}-06$
$-3.2684900 \mathrm{E}-06$
$9.9756262 \mathrm{E}-05$
$2.2914000 \mathrm{E}-06$
$2.0837798 \mathrm{E}-06$
$-3.1944117 \mathrm{E}-07$

C/M
$5.6402220 E-02$
$-1.4065377 E-04$

$-1.4065377 E-04$

$1.9682666 \mathrm{E}-07$

$4.6188670 E-06$

$8.2771369 \mathrm{E}-06$

$-8.9790949 \mathrm{E}-0$

$1.2679213 E-07$

$1.4206552 \mathrm{E}-07$

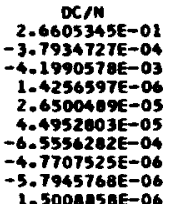

$C / N=N$
$4.2000333 E$

$-2000333 E$
$-1.04736076-02$ $7.9652177 \mathrm{re}-02$ $1.4656063 E-05$ G.1634317e-0 $-6.6002344 E-03$ $-2.3456672 E-05$ $9.4416703 E-0$ $1.0579015 E-05$

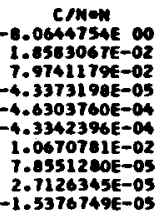

CrMON

$5.1083806 E$ DO $-9.5480701 E-03$ $2.0122001 E-02$ 3.1913254E-0 :-2?5??!IE-04 $-1.0490901 \mathrm{E}-0$. $7.0750156 E-05$ $-5.3710706 \mathrm{E}-03$ $-t-1.40-1,0) \cos \left(-2 L_{1}+5 L_{2}+1 w_{1}-4 w_{2}\right)$

C

1.4. 0 : $1.3407549 E-06 \quad-8.4742498 E-06$

3.4. $0, \quad-9.8742629 E-09 \quad-1.2105117 \mathrm{E}-07$

1.1, 4,1 $\begin{array}{rr}-1.2349246 E-08 & -1.2631749 E-0 \\ B .7314346 E-09 & B .1122395 E-0\end{array}$

$1-2$, s. $\left.0,0, \cos 1-2 L_{1}+5 L_{2}+2 w_{1}-5 w_{2}\right)$

$\begin{array}{ccc}c, 5,0, & C & \text { OC } \\ 4.7968031 E-09 & 4.8634233 E-00\end{array}$

$0^{2}$

$D^{2} \mathrm{C}$

$-6.5832819 E-05$ $-1.5337602 E-06$ $8.9485486 \mathrm{E}-0107$

$-1.0043604$

$-1.0043604 E-04$ $-7.3529434 E-07$ $-9.1959583 E-07$
$6.5019279 E-07$

DCAN

$-6.3104132 E-04$ $-9.0141653 E-06$ $6.0400396 E-06$

crmon

$-7.4790446 E-03$ 5.675\$240E-05 4.8417090E-05
$2,-1,-5,1, \cos \left(-2 L_{1}+5 L_{2}-2 w_{1}+1 w_{2}\right)$

$\begin{array}{ccc}C & C C \\ \text { c 2. } 1.1, & -7.2857556 E-08 & -4.2930423 E-07\end{array}$ $1.8001139 E-10 \quad 3.3936230 \mathrm{E}-09$ \begin{tabular}{llll}
2.3 .1 & $2.8117137 E-09$ & $2.4984761 E-08$ \\
\hline & $1.8677027 E-11$ & $-4.4983448 E-10$
\end{tabular} $1,0,-4,1, \cos 1-2 L_{1}+5 L_{2}-1 w_{1}+\mathrm{ON}_{2}$

$1,0.11 \quad-7.6791344 E-06 \quad-5.1919225 E-05$ 1.0.1 $0.1 \quad-3.4304326 \mathrm{E}-08 \quad-5.6342471 \mathrm{E}-0$ 1.2. 1 2.5423209E-07 $1.1301188 \mathrm{E}-06$ 5. $0.11 \quad-2.1390346 E-10 \quad-3.4386364 E-09$ $\begin{array}{lll}3,2,1 & -1.1936380 E-09 & -2.3357575 E-00 \\ 1,4,1 & -4.9537759 E-09 & -4.2917112 E-08\end{array}$ $1,0.2101 .2181624 E-08 \quad 1.1232375 E-07$ 3. $0.2,2.3838434 \mathrm{E}-10 \quad 3.0317518 \mathrm{E}-09$ $\begin{array}{rrr}1.2,2 & \\ 1.0 .3 & -1.5411718 \mathrm{E}-11 & -1.9213968 \mathrm{~B}-09 \\ & & \end{array}$ $t 0,1,-3,1, \cos \left(-2 L_{1}+5 L_{2}+0 w_{1}-1 w_{2}\right.$

$0.1,11 \quad 1.7138087 E-05 \quad 9.8629426 E-05$ $2,1,1 ;$ (4, 3, 1,1 2.8055787E-07 $-5.8787298 \mathrm{E}-07$ $2.3825420 \mathrm{E}-00$

1 2. 3, 1
$0^{2} \mathrm{C}$

$-3.9166763 E-04$ $-6.3897445 E-06$ $2.1619505 E-06$
$-5.5595875 E-00$ $-4.2255115 E-07$ $-4.9324243 E-07$ $1.1711683 \mathrm{E}-06$ 6.2406044E-O $-2.5594970 E-09$

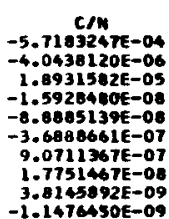

DC/M

-3.8662038E-0 $-4.1955040 E-0$ C.4155136e-0 $-2.56060622-0$ -3.1959sutE-06 6.3642719E-O $2.2576165 E-0$ 2.17543995 $-1.4022252 \mathrm{E}-0 \mathrm{~B}$
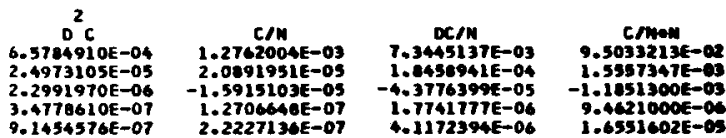

$-4.2381930 \mathrm{x}-02$ $-3.01125476-04$ -1.40975s3e-03 $-6.61609 \cos -06$ $-2.7469416 E-05$ 6.7549495E-05 2.0405622E-07 $-8.5460243 E-00$ 


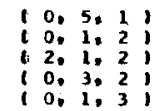

$3.0328431 E-09$

$-9.7200965 \mathrm{E}-10$

$-1.6205411 \mathrm{E}-10$
$2.5610392 \mathrm{E}-11$

$2.6063917 \mathrm{~F}-08$
$-1.8965631 \mathrm{E}-07$

$-1.8965631 \mathrm{E}-07$

$-3.6741989 E-09$

2. $9537892 \mathrm{E}-10$
3. $0595653 \mathrm{E}-07$ $-1.8437100 E-06$ $-1.4791722 E-07$ $3.8418840 \mathrm{E}-09$
2.2584292E-07 $-1.6871882 \mathrm{E}-06$ - $2381423 E-08$ $-1.2067400 E-08$
$1.9408690 \mathrm{E}-06$ $-1.4122898 E-05$ $-8.4254060 \mathrm{E}-07$ $-2.7360196 E-07$ $2.1995611 \mathrm{E}-0 \mathrm{~B}$

$(-1,2,-2,1) \cos \left(-2 L_{1}+5 L+2 L_{2}-2 H\right)$

$\begin{array}{llcc} & & C & C \\ 1,2.1) & -3.5653428 E-07 & -2.6531072 E-06 \\ 3.2,1, & -4.4305906 E-09 & -5.3813804 E-08\end{array}$

(3. 2, 1, $-4.4305906 \mathrm{E}-09 \quad-5.3813804 \mathrm{E}-08$

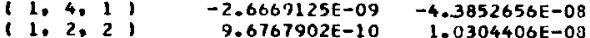

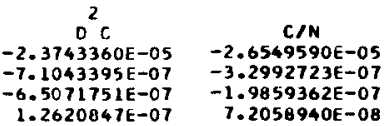

DC/N
$-1.9756560 E-04$
$-4.0072850 E-06$
$-3.2655207 E-06$

$C / N=N$

$-1.9770350 \mathrm{E}-03$

$7.6732529 \mathrm{E}-07$

$1.4789422 E-05$

$5.3659226 \mathrm{E}-06$

(-2. 3, $-1,1) \cos \left(-2 L_{1}+5 L_{2}+2 w_{1}-3 w_{2}\right)$

1.2. 3. 1 1 C DC

$0^{2} \mathrm{C}$

$0 C$
$4.7433529 E-07$

C/N

DC.N

$2.9549562 E-06$

CINAN

2.0803176E-OS

(6, $0,-10,0, \cos \left(-4 \mathrm{~L}_{1}+2 \mathrm{OL} \mathrm{L}_{2}-6 \mathrm{~W}_{1}+\mathrm{OH}_{2}\right)$

(6. $0,0, \quad 4.3925198 \mathrm{C}-08 \quad 4.752 .0448 \mathrm{E}-07$

(8, 0,0$) \quad-3.3384122 \mathrm{E}-10 \quad-3.4388288 \mathrm{E}-09$

$\begin{array}{llll}16,2,0, & -9.2730438 E-09 & -9.7649900 E-08 \\ 16,0,1, & -1.6130533 E-10 & -2.1527894 E-09\end{array}$

$-2.1527894 \mathrm{t}-09$

(5, $1,-9,0) \cos \left(-4 L_{1}+10 L_{2}-5 H_{1}-1 w_{2}\right.$,

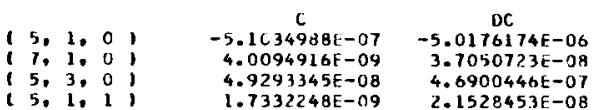

${ }^{2} \mathrm{C}$
$-5.0803241 \mathrm{E}-05$
$3.3913222 \mathrm{~F}-07$
$4.5157475 \mathrm{E}-0.06$

$4.5157475 E-06$

$0^{2} \mathrm{C}$

$5.2651540 t-06$

$-3.5305990 E-08$

$-3.0326411 \mathrm{E}-0 \mathrm{OB}$
C/N
$1.6354613 \mathrm{E}-06$ $-1.2429868 \mathrm{E}-0 \mathrm{OB}$ $-3.4526205 \mathrm{E}-07$ $-6.0058608 \mathrm{E}-09$
DC $/ N$

1.7693228E-05 $-1.2803747 \mathrm{E}-07$ $-3.6357862 \mathrm{E}-06$ $-8.0154532 E-08$
$C / N=N$

6.0892922E-0S $-4.6279967 \mathrm{E}-07$ $-1.2055098 \mathrm{~B}-05$ $-2.2361545 E-07$
1 4, $2,-8, C, \cos \left(-4 L_{1}+10 L_{2}-4 w_{1}-2 w_{2}{ }^{\prime}\right.$

1 4, 2, 0

6. 2,0

$(4,2,1)$

(3, 3, $-7,01$

3. 3, 01

( $5,3,0$,

( $3,3,1$

(2, $4,-6,01$

2. 4.0,

(4. 4,0 ,

$2,6,0$

DC

C

$-2.03064425-08$

$-1.4438923 \mathrm{~F}-07$

$-7.6873801 E-09$

$2.1821095 \mathrm{E}-03$ $-1.6512314 E-07$ $-1.2264700 E-00$ $-8.8454149 t-08$

$\left.\cos 1-4 L+10 L_{2}-3 H_{1}-3 w_{2}\right)$

$C$
$-6.3487841 E-06$
$5.5995250 E-08$

$1.5146197 \mathrm{E}-07$

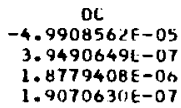

$1.8779408 E-00$

$\cos (-4 L+10 L,-2 w-4 w$

9.1750546F-06

$-9.026+887 \mathrm{E}-08$

OC

OC 17 ? 3E-05

$-5.3249185 \mathrm{E}-07$

$-1.6735233 E-06$
$-2.6008787 E-0 ?$
$-2.3340748 \mathrm{E}-08$

$-2.2691495 E-07$

1 1. 5, -5, 0

$\cos 1-4 L+10 L,-1 \omega,-5 w$

(1, 5, 0

3. 5, 0

1. 7, 0

$-7.057970$

C.

$8.4314951 t-08$

$1.4174152 \mathrm{E}-07$
$1.5483200 \mathrm{E}-08$

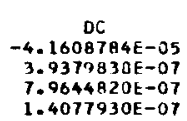

$D^{2} C$
$-2.6755790 E-04$
$1.3755412 E-06$
$4.30945271-06$

$1.4293999 \mathrm{k}-06$
${ }^{2} \mathrm{C}$

$4.1143935 E-04$ $2.6568794 E-06$

$1.4204639 \mathrm{E}-\mathrm{OS}$

$2.2143429 E-06$

\section{${ }^{2} \mathrm{C}$}

$4.6223137 E-04$ $-2.8209910 E=06$ $-1.0885691 E-05$

C/N
$3.4161364 E-04$
$-3.3610072 E-06$ - $3610072 E-06$ $-8.6904308 E-07$

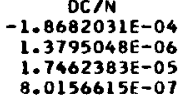

C/NEN

$-1.9001792 \mathrm{E}-05$ $1.4928489 \mathrm{E}-07$ $.0353328 \mathrm{E}-06$
$6.4532937 \mathrm{E}-0 \mathrm{O}$ $8.0156615 E-07$

$-7.0749130 \mathrm{E}-\mathrm{O}$ $5.5583051 E-06$ $6.8334712 E-05$ 2.4027466 E-06
$(0,6,-4,0) \cos \left(-4 L_{1}+10 L_{2}+U w_{1}-6 H_{2}\right)$

$\begin{array}{cccc}\text { ( } 0,6,0, & \text { OC } & \text { 2.2570170F-06 } & 1.1092329 E-05\end{array}$ 2.6.0, $-4.1617028 \mathrm{E}-08 \quad-1.3758498 \mathrm{E}-07$ $\begin{array}{llll}i 0,0,0, & -3.5506052 \mathrm{E}-0 \mathrm{~B} & -1.5387737 \mathrm{E}-07 \\ (0,6,1) & -4.4039483 \mathrm{E}-09 & -3.3414967 \mathrm{E}-08\end{array}$ $-3.3414967 E-08$

\section{$0^{2} \mathrm{c}$}

$6.1818551 E-05$ $-8.7197422 E-0$ $-6.5460561 \mathrm{E}-0$
$-3.3717822 \mathrm{E}-0$

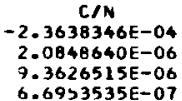

$D C / N$

$-1.8582391 E-03$ $1.4703503 \mathrm{E}-05$ $6.9921131 E-05$ $6.9921131 E-05$
$7.1005431 E-06$
$C / N=N$

$3.4196991 \mathrm{E}-03$ $-2.0016480 E=04$ $-1.0656914 E-05$

$(-1,7,-3,0) \cos \left(-4 L_{1}+10 L_{2}+1 w_{1}-7 w_{2}\right)$

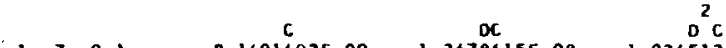
C/N
$-2.6278884 E-04$ $3.1392878 E-06$ $.5008411 E-06$ $5.9137717 E-07$

DC $1 N$

2.3494584E-03 $-1.9826201 \mathrm{E}-0$ $953 E-06$
C/NAN

1.2719257E-02 - 1.2513995e-O $-3.6055638 \mathrm{E}-04$ $-1.0345138 E-07$ C/N
$3.0308080 E-07$ 


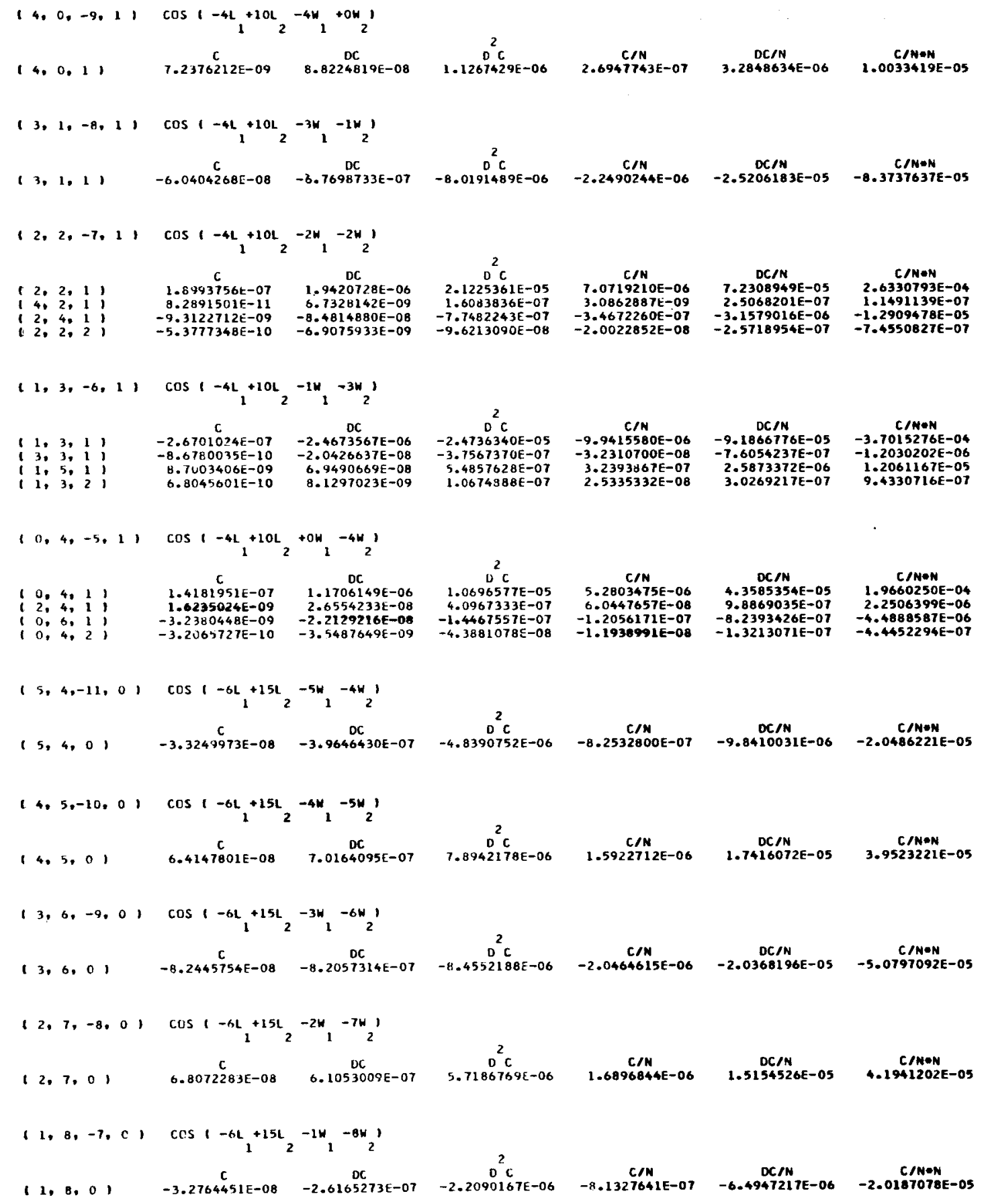


p. 3 In line 5 from bottom, the words "Inner" and "outer" should be transposed.

p. 7 The superscripts $h$ on the right side of equation (16) should read $\mathrm{h}-\mathrm{l}$.

p. 76 The first number of line 4 should read 0.11279093 .

p. 77 The a in line 16 should read + .

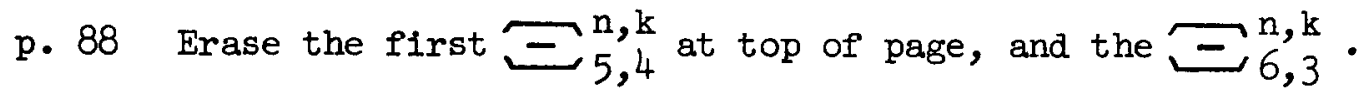

